\title{
Heavier Group 13 Metal(I) Heterocycles Stabilized by Sterically Demanding Diiminophosphinates: A Structurally Characterized Monomer-Dimer Pair For Gallium
}

Andrew L. Hawley, ${ }^{[\mathrm{a}]}$ C. André Ohlin, ${ }^{[\mathrm{a}]}$ Lea Fohlmeister, ${ }^{[\mathrm{a}]}$ Andreas Stasch ${ }^{[\mathrm{a}, \mathrm{b}]}$

Dedicated to Prof. Glen B. Deacon on the occasion of his $80^{\text {th }}$ birthday

${ }^{[a]}$ A. L. Hawley, Dr. C. A. Ohlin, Dr. L. Fohlmeister, Dr. A. Stasch

School of Chemistry, Monash University, 17 Rainforest Walk, Melbourne, Victoria 3800, Australia.

${ }^{[b]}$ Dr. A. Stasch

EaStCHEM School of Chemistry, University of St Andrews, North Haugh, St Andrews, KY16 9ST, United Kingdom.

Email:as411@st-andrews.ac.uk

Supporting information for this article can be found under http://dx.doi.org/10.1002/chem ...

\begin{abstract}
We have synthesized and characterized the monomeric diiminophosphinate-stabilized group 13 metal(I) complexes [ ${ }^{\text {Dip }} \mathrm{LE}:$ ], ${ }^{\mathrm{Dip}} \mathrm{L}=\mathrm{Ph}_{2} \mathrm{P}(\mathrm{NDip})_{2}$, Dip = 2,6-i $\mathrm{Pr}_{2} \mathrm{C}_{6} \mathrm{H}_{3} ; \mathrm{E}=\mathrm{Ga}(\mathbf{1})$, In (2) and $\mathrm{Tl}(\mathbf{3})$. In addition, we structurally characterized the dimeric complex $\left[\left({ }^{\mathrm{Dip}} \mathrm{LGa}\right)_{2}\right], \mathbf{1}_{\mathbf{2}}$. Similar synthetic attempts using ${ }^{\mathrm{Mes}} \mathrm{L}=\mathrm{Ph}_{2} \mathrm{P}(\mathrm{NMes})_{2}$, Mes $=2,4,6-\mathrm{Me}_{3} \mathrm{C}_{6} \mathrm{H}_{2}$ afforded product mixtures from which the mixed oxidation state species $\left[\left({ }^{\mathrm{Mes}} \mathrm{L}\right)_{3} \mathrm{Ga}_{4} \mathrm{I}_{3}\right] \mathbf{4}$ was isolated. [ ${ }^{\mathrm{Dip}} \mathrm{LGa}$ :] $\mathbf{1}$ is converted with dry air to the gallium(III) oxide species $\left[\left({ }^{\mathrm{Dip}} \mathrm{LGaO}\right)_{2}\right] \mathbf{5}$. Density Functional Theory studies on $\left[{ }^{\mathrm{Dip}} \mathrm{LE}:\right]$ and $\left[\left({ }^{\mathrm{Dip}} \mathrm{LE}\right)_{2}\right], \mathrm{E}=\mathrm{Al}-\mathrm{Tl}$, shed light on the bonding in these compounds and show that the newly formed E-E bonding interactions can be described as weak single $\sigma$-bond with no significant $\pi$-bonding contribution for $\mathrm{E}=\mathrm{Al}$, Ga. A large contribution to the dimer binding enthalpies results from London dispersion forces.
\end{abstract}




\section{Introduction}

The past decades have seen enormous advances in the chemistry of molecular low oxidation state compounds of the heavier group 13 metals. ${ }^{[1,2]}$ For $\mathrm{Tl}$, the +1 oxidation state is the most common one ${ }^{[1 b, 3]}$ due to the "inert pair effect", ${ }^{[4]}$ but for the lighter metals Al-In, this oxidation state becomes increasingly difficult to stabilize in molecular compounds. ${ }^{[1,2]}$ This is in turn rewarded with a higher reactivity for the lighter congeners. Thus, there has been significant effort invested in identifying suitable ligand systems, and to developing new synthetic methods to access stable and well-defined complexes with the lighter group 13 metals in the +1 oxidation state. A prominent subclass of these are stable monomeric heterocycles, ${ }^{[1,2]}$ and notable examples are highlighted in Figure 1 . These include the most widely investigated $\beta$-diketiminate examples of $\mathrm{Al}^{[5]}{ }^{[5]} \mathrm{Ga},{ }^{[6]}$ and $\operatorname{In}^{[7]}(\mathbf{A})$, anionic five-membered heterocycles $(\mathbf{B})$ for gallium, ${ }^{[6 a, 8]}$ that are valence-isoelectronic to important imidazolylidene-NHCs, ${ }^{[9]}$ and four-membered guanidinate-stabilized heterocycles (C) for gallium and indium. ${ }^{[10]}$ In addition, different classes of ligands with different steric and electronic profiles have allowed the isolation and study of unusual molecular compounds with low oxidation state group 13 metals from low-coordinate mononuclear species to giant cluster compounds. ${ }^{[1,2]}$

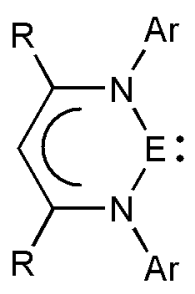

A<smiles>[R]C1=C([R])N([R7])[Ga]N1[Z17]</smiles>

B<smiles></smiles>

C

$$
E=G a, \ln
$$

$\mathrm{E}=\mathrm{Al}, \mathrm{Ga}, \mathrm{In}$

$\mathrm{R}=$ selected alkyls, $\mathrm{Ar}=$ sterically demanding aryls, $\mathrm{R}^{1}=\mathrm{H}$, alkyl

$\mathrm{R}^{1}{ }_{2}=$ acetenaphtenediyl, $\mathrm{R}^{2}=\mathrm{Ar}$ or $t \mathrm{Bu}$, Dip $=2,6-\mathrm{Pr}_{2} \mathrm{C}_{6} \mathrm{H}_{3}$

Figure 1. Metal(I) N-heterocycles of Al-In.

Apart from their fundamental importance, the molecules of the lighter metal(I) ions and related low oxidation state species are also highly reactive and can reduce numerous substrates and activate relatively inert bonds. ${ }^{[1,2]}$ This is generally due to a high energy electron configuration that often involves a filled metal-based orbital (often the HOMO), and the accessibility of vacant metal orbital(s) such as the LUMO or nearby orbitals. The heterocyclic variants of these molecules that accommodate a lone pair of electrons on their group 13 metal centre such as A-C, have thus been coined group 13 carbene analogues due to their electronic similarities with $N$-heterocyclic 
carbenes. ${ }^{[9]}$ Consequently, these species have also been widely studied for their coordination chemistry to (transition) metal fragments. ${ }^{[1,2]}$ Here we report on the outcomes of reactions of diiminophosphinato alkali metal complexes with inorganic group 13 metal(I) sources.

\section{Results and Discussion}

\section{Synthesis and Characterization}

We have previously prepared the sterically demanding diiminophosphinate ligand $\left[\mathrm{Ph}_{2} \mathrm{P}(\mathrm{NDip})_{2}\right]^{-}$, ${ }^{\text {Dip }} \mathrm{L}^{-}$, Dip $=2,6-i \mathrm{Pr}_{2} \mathrm{C}_{6} \mathrm{H}_{3},{ }^{[11,12]}$ and used it to stabilize highly reactive low oxidation state complexes of zinc(I) ${ }^{[11]}$ and magnesium(I). ${ }^{[13]}$ These highly reducing complexes are stable despite the high oxidation state iminophosphorane centres $\left(\mathrm{P}^{\mathrm{V}}\right)$ and the low oxidation state metals ions. We therefore also investigated the ligands' ability to stabilize related low oxidation state group 13 metal complexes.

Reacting alkali metal complexes of ${ }^{\mathrm{Dip}} \mathrm{L}^{-},\left[{ }^{\mathrm{Dip}} \mathrm{LM}\right](\mathrm{M}=\mathrm{Li}, \mathrm{Na}, \mathrm{K}),{ }^{[11,12]}$ with the metal(I) sources "GaI"[14,15], InBr, or TlBr, respectively, afforded the group 13 metal(I) complexes [ ${ }^{\text {Dip }} \mathrm{LE}$ :] $(\mathrm{E}=\mathrm{Ga}$ 1, In 2, Tl 3) in moderate to good yields as typically colourless crystalline solids, see scheme 1.

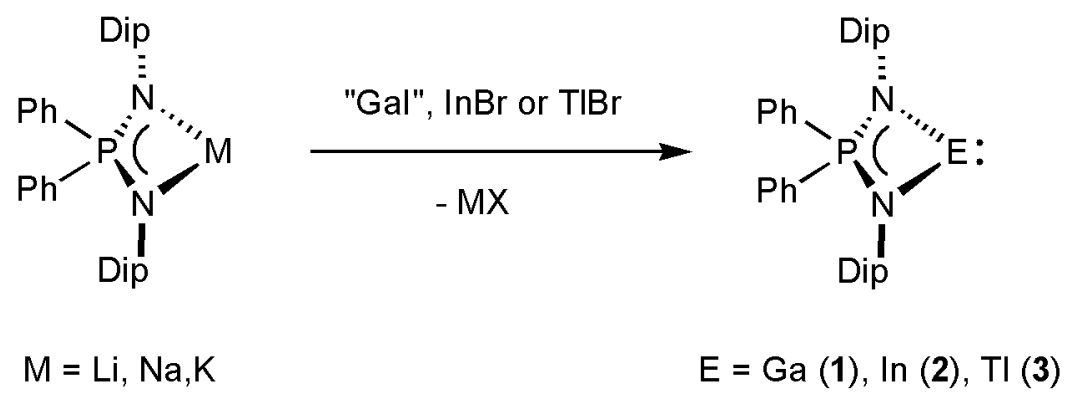

Scheme 1. Synthesis of Compounds 1-3.

Reactions involving the mixed oxidation state solid "GaI" to prepare [ ${ }^{\mathrm{Dip}} \mathrm{LGa}$ :] $\mathbf{1}$ had to be performed in aromatic solvents such as toluene. The reactions to synthesize [ $\left.{ }^{\mathrm{Dip}} \mathrm{LIn}:\right] \mathbf{2}$ and $\left[{ }^{\mathrm{Dip}} \mathrm{LTl}\right.$ :] 3 were best performed using finely powdered metal(I) salts in THF using a low temperature route. All alkali metal complexes [ $\left.{ }^{\mathrm{Dip}} \mathrm{LM}\right](\mathrm{M}=\mathrm{Li}, \mathrm{Na}, \mathrm{K})$ were used to obtain the desired complexes 1-3, although in line with the molecular structures, aggregation states, and resulting solubilities of the alkali metal complexes, ${ }^{[12]}$ as well as the formation and precipitation of alkali metal halide byproducts, some combinations were preferred. For the synthesis of $\left[{ }^{\mathrm{Dip}} \mathrm{LGa}\right.$ :] $\mathbf{1}$ in toluene, $\left[{ }^{\mathrm{Dip}} \mathrm{LLi}\right]$ and $\left[{ }^{\text {Dip }} \mathrm{LNa}\right]$ are suitable salt metathesis precursor complexes. Both $\left[{ }^{\mathrm{Dip}} \mathrm{LLi}\right]$ and $\left[{ }^{\mathrm{Dip}} \mathrm{LNa}\right]$ show dimeric structures in the solid state and are soluble in aromatic solvents, with the lithium complex being the more soluble one. The formation and precipitation of $\mathrm{NaI}$ was preferred in comparison 
with LiI, and the use of the sodium complex afforded reliable preparation of $\mathbf{1}$. The syntheses of [ ${ }^{\text {Dip }}$ LIn:] 2 and [ ${ }^{\text {Dip }}$ LTl:] 3 were conveniently obtained using $\left[{ }^{\text {Dip }} \mathrm{LNa}\right.$ or $\left[{ }^{\text {Dip }} \mathrm{LK}\right]$ in THF using low temperature approaches.

The complexes [ ${ }^{\text {Dip }} \mathrm{LE}:$ ] ( $\mathrm{E}=\mathrm{Ga} \mathbf{1}$, In 2, Tl 3) could be crystallized from a range of hydrocarbon and ether solvents. All three complexes could be obtained with solvent of crystallization as colourless crystals in the tetragonal crystal system with half a molecule in the asymmetric unit, see Figure 2, and Table 1 for metrical data. Complexes 1-3 are monomeric with $N, N^{\prime}$-chelating dimiinophosphinate ligands coordinating to a univalent group 13 metal ion. The Ga$\mathrm{N}$ and In-N bond lengths lie in the expected range for such interactions, for example as those found in $\mathbf{A}$ and $\mathbf{C}$. A molecular structure of the thallium complex $\mathbf{3}$ was not obtained with sufficient quality and appears to be poorly ordered from a variety of solvents, but the overall connectivity is unambiguous. Thallium(I), and to a lesser extend indium(I), complexes of related sterically demanding guanidinates and amidinates ${ }^{[10,16]}$ show $N$, Aryl-coordination modes, compared with $N, N^{\prime}$-chelating ones found in 1-3, presumably because the $\mathrm{N} \cdots \mathrm{N}$ separation in amidinates/guanidinates is slightly smaller when compared with diiminophosphinates. This $\mathrm{N} \cdots \mathrm{N}$ separation is, for example, ca. 0.23-0.25 $\AA$ larger for $\mathbf{1}$ and $\mathbf{2}$ compared with related guanidinate examples of $\mathrm{Ga}$ and In (C), but is as expected considerably shorter (by ca. $0.4 \AA$ ) than those of the six-membered chelates (A) and those of the five-membered derivatives (B) (by ca. 0.1-0.2 $⿱$ ). In the packing of the tetragonal structures of 1-3, two molecules are located in one plane and their metal centers are oriented toward each other with approximate $\mathrm{M} \cdots \mathrm{M}$ separations of $4.94 \AA(\mathrm{Ga})$, $4.54 \AA$ (In) and $4.14 \AA$ (Tl) (e.g. see Figure S3). The voids in-between the molecules are partially filled with highly disordered solvent molecules and the same packing was found for several different solvents.

A different isomer, off-white to light yellow dimeric $\left[\left({ }^{D i p} L G a\right)_{2}\right] \cdot \mathrm{C}_{6} \mathrm{H}_{6}, \mathbf{1}_{2} \cdot \mathrm{C}_{6} \mathrm{H}_{6}$, was crystallized from benzene with a full monomer in the asymmetric unit, see Figure 2 and Table 1. In this molecular structure, two ${ }^{\text {Dip }}$ LGa units are arranged in the solid state with co-planar heterocycles, a Ga-Ga bonding contact of $c a .2 .79 \AA$ and a P...Ga-Ga angle of $c a .110^{\circ}$. The Ga-N distances in $\mathbf{1}_{2}$ are slightly contracted and the P-N bonds slightly elongated in comparison with those of monomeric 1, see Table 1. Previously, few diiminophosphinate complexes of group 13 elements in the +3 oxidation state have been reported. ${ }^{[17]}$ To the best of our knowledge, no structurally characterized iminophosphorane complexes of low oxidation state group 13 metals have been reported, but an indium(II) and a thallium(I) complex of an amidophosphazane ligand are published. ${ }^{[18]}$

So far, no neutral heterocyclic gallium(I) dimer has been prepared, though some structurally authenticated dimeric compounds of the anionic five-membered Ga species $\mathbf{B}$ with bridging and 
interacting potassium countercations are reported ${ }^{[8 \mathrm{e}]}$ that show $\mathrm{Ga} \cdots \mathrm{Ga}$ separations of $c a$. 2.86$2.88 \AA$ and a similar overall ligand-gallium orientation to $\mathbf{1}_{\mathbf{2}}$. For indium(I), some of the known $\beta$ diketiminate examples show weak and long In $\cdots$ In interactions in the solid state $(\geq 3.2 \AA)$ with an approximate ligand plane-In $\cdots$ In angle of $113^{\circ},{ }^{[7]}$ whereas others are monomeric. It has been concluded that the In *In bonding is very weak (within a few $\mathrm{kcal} / \mathrm{mol}$ ), and it has been shown that they are monomeric in the solution phase. A computational study on $\beta$-diketiminate metal(I) dimers of $\mathrm{Al}, \mathrm{Ga}$ and In supports stronger bonding for the lighter metals. ${ }^{[19]}$ One other related and remarkable $\beta$-diketiminate In complex has been reported, $\left[\left({ }^{\mathrm{X}} \mathrm{LInI}\right)_{2}\left({ }^{\mathrm{X}} \mathrm{LIn}\right)_{4}\right]\left({ }^{\mathrm{X}} \mathrm{L}=\mathrm{HC}\{\mathrm{MeCN}(3,5\right.$ $\left.\left.\mathrm{Me}_{2} \mathrm{C}_{6} \mathrm{H}_{3}\right)\right\}_{2}$ ), that features a $\sigma$-bonded catenated $\mathrm{In}_{6}$ chain having relatively short In-In bonds of $c a$. 2.84-2.85 $\AA$ between indium(I) centres, that suggest a different type of bonding with $\mathrm{sp}^{3}$-like hybridization on In. ${ }^{[20]}$ More akin to the gallium-gallium interaction in $\mathbf{1}_{\mathbf{2}}$, several sterically demanding substituted 2,6-terphenyl gallium(I) complexes have been described, that can show both monomeric and dimeric solid state structures and thus contain rare one-, and two-coordinate Ga centres, respectively. ${ }^{[21]}$ Complementary computational studies show that these interactions have to be considered as weak and were strongly dependent on the employed substituted terphenyl substituents. Bond lengths in these ArGaGaAr molecules range from $c a$. 2.51-2.63 $\AA$ and further longer, calculated interactions were determined. The Ga-Ga bond lengths depend on the geometry of the interaction and the maximum bond strength was calculated to be around $20-38 \mathrm{~kJ} / \mathrm{mol}$ depending on the substitution pattern of the bulky ligands.
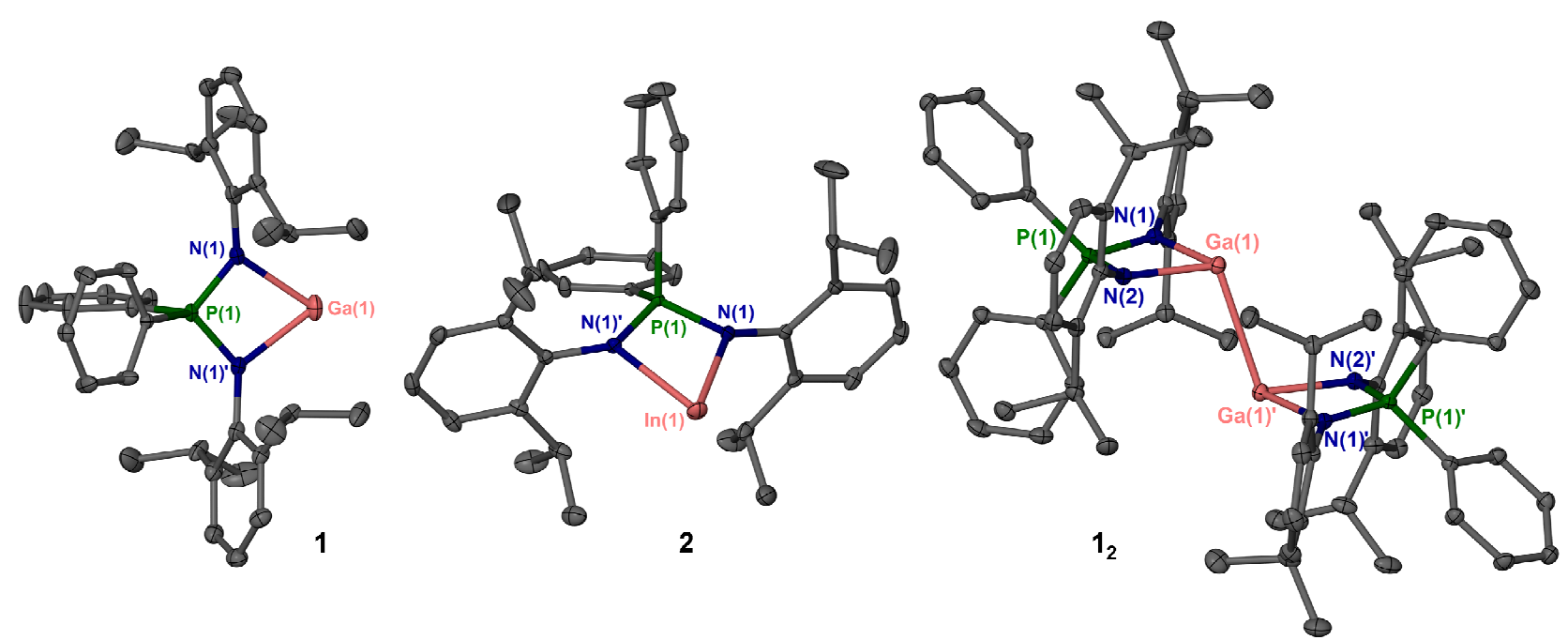

Figure 2. Molecular structures of compounds 1-3 (30\% thermal ellipsoids). Hydrogen atoms, lattice solvents and minor parts of disordered isopropyl groups omitted for clarity. 
As expected, complexes 1-3 show NMR resonances that support a highly symmetric averaged geometry in solution. For example, one doublet and one septet only are found in the ${ }^{1} \mathrm{H}$ NMR spectra of 1-3 in deuterated benzene, as was previously determined for homoleptic metal(I) complexes of ${ }^{\mathrm{Dip}} \mathrm{L}^{-[11-13]}$ The complexes show resonances at $\delta 16.4 \mathrm{ppm}(\mathrm{E}=\mathrm{Ga}), \delta 7.35$ (In) and $\delta$-3.7 (Tl) that shift upfield for the heavier elements and towards resonances for the ionic alkali metal complexes of this ligand, ${ }^{[12]}$ and may be suggestive of a more ionic ligand-metal interaction for thallium(I) species 3. The ${ }^{31} \mathrm{P}\left\{{ }^{1} \mathrm{H}\right\}$ NMR resonance for $\mathbf{1}$ is a broad singlet, a sharp singlet for $\mathbf{2}$, and a broad doublet with ${ }^{2} J_{\mathrm{TIP}}$ coupling of $c a .416 \mathrm{~Hz}$ for the combined ${ }^{205 / 203} \mathrm{Tl}$ nuclei. Dissolving crystalline off-white to light yellow $\mathbf{1}_{2}$ in deuterated benzene led to dissolution with concomitant decolourization and afforded identical solution NMR spectra to those of $\mathbf{1}$, suggesting the dissociation into monomers. The indium derivative $\mathbf{2}$ is significantly less thermally stable compared with their Ga (1) and Tl (3) counterparts both in the solid and solution state. Decomposition of 2 yields In metal and the proligand ${ }^{\mathrm{Dip}} \mathrm{LH}$.

Table 1. Selected interatomic distances $(\AA)$ and angles $\left(^{\circ}\right)$ for complexes $\mathbf{1}, \mathbf{1}_{2}$ and $\mathbf{2}$.

\begin{tabular}{|c|c|c|c|}
\hline & $\mathrm{E}=\mathrm{Ga} 1$ (monomer) & $\mathrm{E}=\mathrm{Ga} \mathbf{1}_{2}($ dimer $)$ & $\mathrm{E}=\mathrm{In} 2$ (monomer) \\
\hline $\mathrm{E}-\mathrm{N} / \AA$ & $2.130(3)$ & $2.071(2), 2.087(2)$ & $2.340(2)$ \\
\hline $\mathrm{P}-\mathrm{N} / \AA$ & $1.601(3)$ & $1.610(2), 1.612(2)$ & $1.605(2)$ \\
\hline $\mathrm{E} \cdots \mathrm{E}$ or $\mathrm{E}-\mathrm{E} / \AA$ & $\begin{array}{l}\text { ca. } 4.94 \text { (separation in } \\
\text { lattice) }\end{array}$ & $2.7873(12)$ & $\begin{array}{l}\text { ca. } 4.54 \text { (separation in } \\
\text { lattice) }\end{array}$ \\
\hline $\mathrm{P} \cdots \mathrm{E}-\mathrm{E} /^{\circ}$ & - & $109.8^{\circ}$ & - \\
\hline
\end{tabular}

To study the influence of the ligand sterics on the system, we reacted alkali metal complexes of the mesityl-substituted diiminophosphinate ${ }^{\mathrm{Mes}} \mathrm{L}^{-},\left[{ }^{\mathrm{Mes}} \mathrm{LM}\right],{ }^{\mathrm{Mes}} \mathrm{L}=\mathrm{Ph}_{2} \mathrm{P}(\mathrm{NMes})_{2}$, Mes = mesityl, 2,4,6$\mathrm{Me}_{3} \mathrm{C}_{6} \mathrm{H}_{2} ;{ }^{[12]}$ with the same group 13 metal(I) halide sources. One anticipated possible outcome would be the synthesis of further dimeric metal(I) complexes with a different stability. These reactions, however, gave mixtures of products and we were so far unable to isolate pure compounds. Especially for the gallium system, ${ }^{31} \mathrm{P}\left\{{ }^{1} \mathrm{H}\right\} \mathrm{NMR}$ spectroscopic analysis of the reaction mixture revealed resonances for numerous ligand environments suggesting that a mixture of many products had formed. From these mixtures, we obtained few colourless rods that were crystallographically characterized as $\left[\left({ }^{\mathrm{Mes}} \mathrm{L}\right)_{3} \mathrm{Ga}_{4} \mathrm{I}_{3}\right] \mathbf{4}$, see Figure 3. The molecule crystallized from $n$-hexane with half a molecule in the asymmetric unit and shows relatively large libration modes on the organic substituents, but the central $\mathrm{Ga}_{4} \mathrm{I}_{3}$ core is well ordered. In 4, a central gallium atom $(\mathrm{Ga} 2)$ is bonded to three $\left\{\left({ }^{\mathrm{Mes}} \mathrm{L}\right) \mathrm{Ga}\right\}$-fragments and $\mathrm{I} 2$ in a distorted tetrahedral fashion. The geometry around the Ga2-Ga1 bonded $(2.45 \AA)$ unit to the $\left\{\left({ }^{\mathrm{Mes}} \mathrm{L}\right) \mathrm{Ga}(\mathrm{I})\right\}$-fragment is a common structural motif found for gallium(II) complexes of the type $\mathrm{LGa}(\mathrm{X})-\mathrm{Ga}(\mathrm{X}) \mathrm{L}$, where $\mathrm{L}$ is a sterically 
demanding $N, N^{\prime}$-chelating ligand. ${ }^{[10 b, 22]}$ Two further $\left\{\left({ }^{\mathrm{Mes}} \mathrm{L}\right) \mathrm{Ga}\right\}$-fragments $(\mathrm{Ga} 3, \mathrm{Ga} 3$ ') bind to Ga2 in an almost linear fashion ( $\mathrm{P} \cdots \mathrm{Ga} 3-\mathrm{Ga} 2: 164^{\circ}$ ) and a remaining iodide ligand (I3) bridges both $\left\{\left({ }^{\mathrm{Mes}} \mathrm{L}\right) \mathrm{Ga}\right\}$ centers at an almost right angle $\left(\mathrm{Ga} 2-\mathrm{Ga} 3-\mathrm{I3}: 92^{\circ}\right)$. These latter structural features suggest a simplified bonding description of lone pair donation of two $\left\{\left({ }^{\mathrm{Mes}} \mathrm{L}\right) \mathrm{Ga}^{\mathrm{I}}\right\}$ fragments $(\mathrm{Ga} 3$, Ga3') to the cationic $\mathrm{Ga}(\mathrm{I})-\mathrm{Ga}(\mathrm{I})\left({ }^{\mathrm{Mes}} \mathrm{L}\right)$ moiety via $\mathrm{Ga}$, and coordination of one iodide (I3) into empty p-orbitals of both of the coordinating $\left\{\left({ }^{\mathrm{Mes}} \mathrm{L}\right) \mathrm{Ga}^{\mathrm{I}}\right\}$ groups $\left(\mathrm{Ga} 3, \mathrm{Ga} 3{ }^{\prime}\right)$. This results in $\sigma$ bonding interactions between $\mathrm{Ga} 2$ and the other $\mathrm{Ga}$ atoms and an average formal gallium oxidation state of +1.5 . For reactions of the respective indium system, an X-ray diffraction study suggested that the indium(III) complex $\left[\left({ }^{\mathrm{Mes}} \mathrm{L}\right)_{2} \mathrm{InBr}\right]$ is part of the product mixture.

Similar structural features to $\left[\left({ }^{\mathrm{Mes}} \mathrm{L}\right)_{3} \mathrm{Ga}_{4} \mathrm{I}_{3}\right] 4$ were previously found and comparable bonding explanations have been given for a related, slightly larger, branched $\mathrm{Ga}_{5}$ molecule $\left[\mathrm{L}_{3} \mathrm{Ga}_{5} \mathrm{I}_{4}\right]\left(\mathrm{L}=\mathrm{HC}\{\mathrm{C}(t \mathrm{Bu}) \mathrm{NMes}\}_{2}\right)$ with an average gallium oxidation state of $+1.4,{ }^{[6 \mathrm{a}]}$ and a mixed-valent amido gallium complex with a bridging iodide ligand. ${ }^{[23]}$ In the former case, this branched molecule was a by-product alongside the main product [LGa:], a type A heterocyclic complex. As with 4, this system also features mesityl substituents on the nitrogen atoms, in contrast to highly successful N-Dip groups as used in $\mathbf{1}$ and in the majority of stable compounds of type AC. Previously it has been found, that the synthesis and stabilization of closely related guanidinate stabilized metal(I) heterocycles of gallium and indium (type $\mathbf{C}$ ) was highly dependent on the steric bulk of the ligand and minor changes to the ligand periphery led to different products or mixtures with metal centres in different oxidation states. ${ }^{[10,16]}$

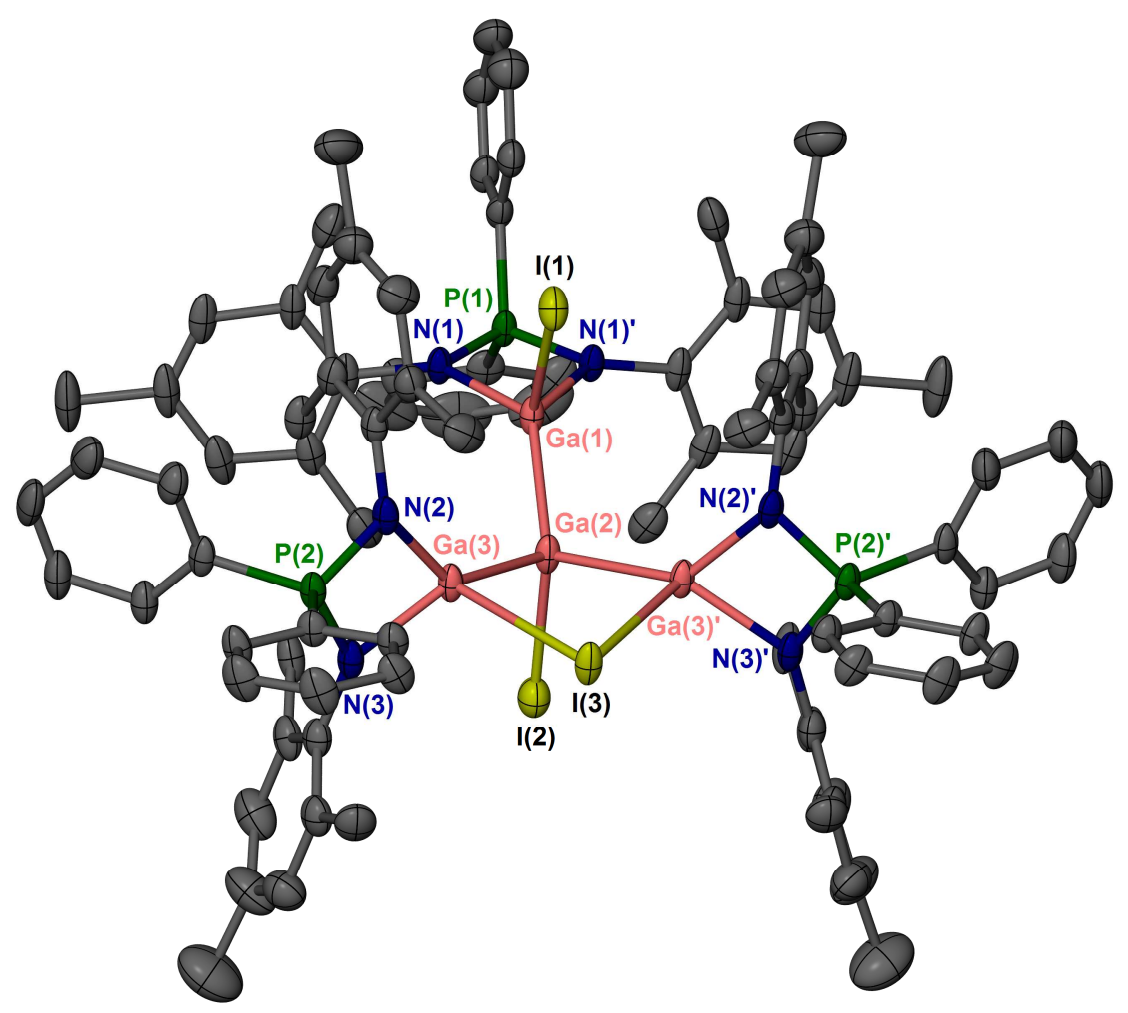


Figure 3. Molecular Structures of Complex $\left[\left({ }^{\mathrm{Mes}} \mathrm{L}\right)_{3} \mathrm{Ga}_{4} \mathrm{I}_{3}\right] 4$ (30\% thermal ellipsoids). Selected bond lengths $(\AA)$ and angles $\left(^{\circ}\right)$. I(1)-Ga(1) 2.6326(12), I(2)-Ga(2) 2.6214(13), I(3)-Ga(3) 2.8829(7), $\mathrm{Ga}(1)-\mathrm{Ga}(2)$ 2.4506(12), $\mathrm{Ga}(2)-\mathrm{Ga}(3)$ 2.4712(8), $\mathrm{Ga}(1)-\mathrm{N}(1)$ 1.988(4), N(2)-Ga(3) 1.976(5), Ga(3)-N(3) 2.010(4), P(1)-N(1) 1.625(4), P(2)-N(2) 1.632(4), P(2)-N(3) 1.626(5); Ga(1)-

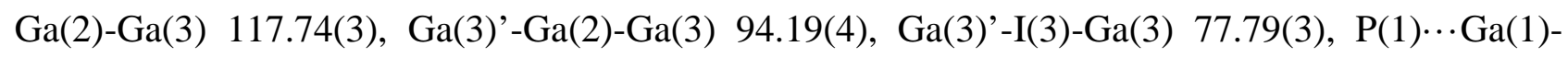
$\mathrm{Ga}(2)$ 148.6, $\mathrm{P}(2) \cdots \mathrm{Ga}(3)-\mathrm{Ga}(2)$ 163.6.

\section{Properties and Reactivity}

The lightest synthesized congener $\mathbf{1}$ is the most promising candidate for reactivity studies. We noticed in an initial crystal structure determination of the dimer $\left[\left({ }^{\mathrm{Dip}} \mathrm{LGa}\right)_{2}\right], \mathbf{1}_{\mathbf{2}}$, that a small percentage could be attributed to the gallium(III) oxide compound [ $\left.\left({ }^{\mathrm{Dip}} \mathrm{LGaO}\right)_{2}\right] \mathbf{5}$ with a similar overall geometry, though with a slightly smaller metal ion and possessing bridging oxide ligands. We therefore treated [ ${ }^{\mathrm{Dip}} \mathrm{LGa}$ :] $\mathbf{1}$ with dry air on a preparative scale, and in a small scale experiment followed by NMR spectroscopy, and isolated the oxide $\left[\left({ }^{\mathrm{Dip}} \mathrm{LGaO}\right)_{2}\right] \mathbf{5}$ in moderate yield as a colourless crystalline solid, see Scheme 2 and Figure 3. Complex $\left[\left({ }^{\mathrm{Dip}} \mathrm{LGaO}\right)_{2}\right] \mathbf{5}$ contains two bridging oxide ligands between two ${ }^{\text {Dip }} \mathrm{LGa}$ units that form a square four-membered $\mathrm{Ga}_{2} \mathrm{O}_{2}$ ring perpendicular to the co-planar ligand-gallium planes. Previously, gallium(I) complexes could be converted to galloxane species, namely the $\beta$-diketiminate derivative $\left[\left(\mathrm{HC}\{\mathrm{C}(\mathrm{Me}) \mathrm{N}(\mathrm{Dip})\}_{2} \mathrm{GaO}\right)_{2}\right]^{[24]}$ and the diazabutadienediide derivative $[\mathrm{K}(\mathrm{TMEDA})]_{2}\left[\left(\left\{(\mathrm{HCN}(\mathrm{Dip})\}_{2} \mathrm{GaO}\right)_{2}\right]\left(\mathrm{TMEDA}=N, N, N^{\prime}, N^{\prime}\right.\right.$-tetramethylethylenediamine $),{ }^{[25]}$ that were obtained from the respective gallium(I) synthons with $\mathrm{N}_{2} \mathrm{O}$. For the synthesis of the latter complex, stoichiometric $\mathrm{N}_{2} \mathrm{O}$ had to be employed and the reaction of its precursor with air led to decomposition and formation of the free diazabutadiene proligand $\left\{(\mathrm{HC}=\mathrm{N}(\mathrm{Dip})\}_{2}\right.$. The $\mathrm{Ga}-\mathrm{O}$ bond lengths in $\mathbf{5}$ are $c a .1 .82 \AA$ and $1.85 \AA$, respectively; the latter value being identical to those found for the $\beta$-diketiminate derivative $\left[\left(\mathrm{HC}\{\mathrm{C}(\mathrm{Me}) \mathrm{N}(\mathrm{Dip})\}_{2} \mathrm{GaO}\right)_{2}\right]$. The $\mathrm{Ga}-\mathrm{N}$ bond lengths in $\mathbf{5}$ are $c a$. $1.97 \AA-1.98 \AA$, and identical to those found in $\left[\left(\mathrm{HC}\{\mathrm{C}(\mathrm{Me}) \mathrm{N}(\mathrm{Dip})\}_{2} \mathrm{GaO}\right)_{2}\right]$, and are $c a$. $0.1 \AA$ shorter than in the starting material 1 due to the oxidation to the smaller and harder $\mathrm{Ga}^{3+}$ cation in $\mathbf{5}$. Exposure of compound $\mathbf{2}$ to air only leads to decomposition and the precipitation of indium metal.
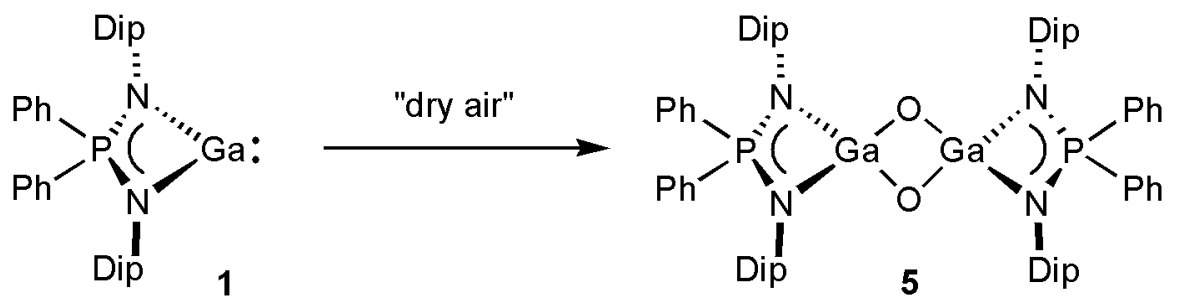

Scheme 2. Synthesis of compound 5 from $\mathbf{1}$. 
Compounds 1-3 do not react with the strong and small N-donor DMAP (4-dimethylaminopyridine). No significant changes are found to solution NMR spectra of compounds 1-3 with an excess of DMAP, and uncoordinated 1-3 could be partially recrystallized. This is somewhat surprising given the two-coordinate metal centres with a vacant orbital (vide infra). Aromatic $\mathrm{N}$-donors can form $\sigma$ donor bonds to species such as gallium(I) cations, ${ }^{[26]}$ and the first well-characterized monomeric gallium(I) complex stabilized by a tris(pyrazolyl)borate ligand shows three equal $\mathrm{N}$-coordination bonds to gallium(I). ${ }^{[27]}$ Compound $\mathbf{1}$ could also be crystallized directly from the donor solvent THF at low temperatures which could be made use of for extraction purposes from reaction mixture residues.

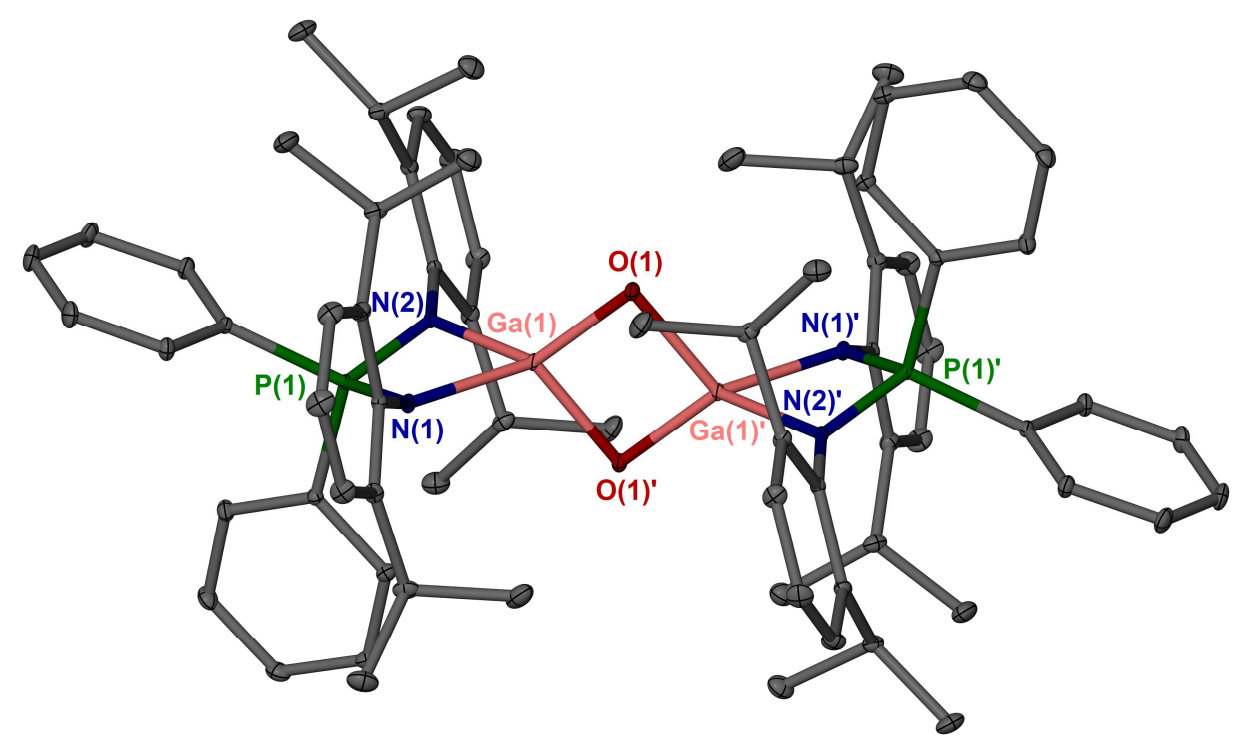

Figure 4. Molecular structures of complex $\left[\left\{\left({ }^{\mathrm{Dip}} \mathrm{L}\right) \mathrm{GaO}\right\}_{2}\right]$ 5. Selected bond lengths $(\AA)$ and angles $\left(^{\circ}\right) . \mathrm{Ga}(1)-\mathrm{O}(1)$ 1.8149(8), Ga(1)-O(1)’ 1.8517(8), Ga(1)-N(1) 1.9672(8), Ga(1)-N(2) 1.9757(9), $\mathrm{Ga}(1) \cdots \mathrm{Ga}(1)$ ' 2.5354(2), P(1)-N(1) 1.6221(9), P(1)-N(2) 1.6285(9); O(1)-Ga(1)-O(1)’ 92.51(3), $\mathrm{Ga}(1)-\mathrm{O}(1)-\mathrm{Ga}(1)^{\prime} 87.49(3)$.

\section{Computational Studies}

The full monomers $\left[{ }^{\text {Dip }} \mathrm{LE}:\right]\left(\mathrm{E}=\mathrm{Al}, \mathrm{Ga}\right.$ 1, In 2, Tl 3) and the respective dimers $\left[\left({ }^{\mathrm{Dip}} \mathrm{LE}\right)_{2}\right]$, were optimised using Density Functional Theory (DFT) studies mainly at the pbe0/def2-tzvp(E,N,P), def2-svp $(\mathrm{C}, \mathrm{H})$ level of theory (see the Supporting Information for full details). The monomers and dimers were optimized in their singlet states, and the triplet states for the monomers were also optimized for comparison. Selected data is summarized in Table 2 and the optimized geometries of $\left[\left({ }^{\mathrm{Dip}} \mathrm{LE}\right)_{2}\right]$ are shown in Figure S4.

The experimental geometries of the monomeric [ $\left.{ }^{\mathrm{Dip}} \mathrm{LE}:\right]$ species are reproduced well with good to excellent agreement of key bond distances. The optimized dimer molecules show relatively 
symmetric structures with the exception of the more distorted $\mathrm{Tl}$ dimer molecule. This may be due to the relatively small ligand bite-angle in combination with a large metal ion and predicted weak $\mathrm{Tl} \cdots \mathrm{Tl}$ bonding. There is good agreement between the calculated and experimentally found geometries of $\left[\left({ }^{\mathrm{Dip}} \mathrm{LGa}\right)_{2}\right] \mathbf{1}_{\mathbf{2}}$. Geometry optimization of the dimerized heterocycles led to no significant structural changes in the monomeric units in comparison with the isolated monomers, albeit a slight contraction of the E-N bond lengths was observed despite a higher metal coordination number that was also found in the pair of crystal structures of $\mathbf{1}$ and $\mathbf{1}_{\mathbf{2}}$. The calculated P-N bond lengths decrease down the group presumably due to an increased ionic bonding character between the ${ }^{\text {Dip }} \mathrm{L}^{-}$ligand and $\mathrm{E}^{+}$group 13 metal(I) ion and along with a lower Lewis acidity of $\mathrm{Tl}^{+}$compared with $\mathrm{Al}^{+}$. Only very small differences in the P-N bond lengths of respective monomer-dimer pairs are found for the optimized geometries and their significance has to be viewed with caution. There is, however, a small but significant difference in the pair of solid state structures of $\mathbf{1}$ and $\mathbf{1}_{2}$ (Table 1), with the dimer molecule producing the larger P-N bond lengths similar to the trend found from the computations. Both the HOMO-LUMO energy gaps and the singlet-triplet energy gaps of the monomers [ $\left.{ }^{\mathrm{Dip}} \mathrm{LE}:\right]$ are increasing down the group. 
Table 2. Selected computational (DFT) results for the optimized monomers and dimers of [ ${ }^{\mathrm{Dip}} \mathrm{LE}$ :], $\mathrm{E}=\mathrm{Al}, \mathrm{Ga}$, In, Tl. Details are given at the pbe0/def2-tzvp(E,N,P), def2-svp(C,H) level, except where noted. GD3-BJ indicates the D3 version of Grimme's dispersion with Becke-Johnson damping, as applied to pbe 0 . LP denotes lone pair.

\begin{tabular}{|c|c|c|c|c|}
\hline & $\mathrm{E}=\mathrm{Al}$ & $\mathrm{E}=\mathrm{Ga}$ & $\mathrm{E}=\mathrm{In}$ & $\mathrm{E}=\mathrm{Tl}$ \\
\hline E-N (mono)/A & 2.003 & 2.116 & 2.335 (mean) & 2.457 (mean) \\
\hline E-N (dimer)/Å & 1.985 (mean) & 2.093 (mean) & 2.310 (mean) & 2.439 (mean) \\
\hline $\mathrm{P}-\mathrm{N}(\mathrm{mono}) / \AA$ & 1.616 & 1.610 & 1.607 (mean) & 1.603 \\
\hline $\mathrm{P}-\mathrm{N}($ dimer $) / \AA$ & 1.619 (mean) & 1.613 (mean) & 1.609 (mean) & 1.604 (mean) \\
\hline E-E/Å & 2.762 & 2.830 & 3.289 & 3.662 \\
\hline E-E/Å (GD3-BJ) & 2.679 & 2.689 & 3.083 & 3.275 \\
\hline $\mathrm{P} \cdots \mathrm{E}-\mathrm{E}($ dimer$) /^{\circ}$ & 117.5 (mean) & 114.0 (mean), & 111.4 (mean) & 110.3 mean \\
\hline $\begin{array}{l}\triangle E_{\text {Номо-LUMO- }} \\
{ }_{\text {mопо }} / \mathrm{kJ} / \mathrm{mol}(\mathrm{eV})\end{array}$ & $333(3.45)$ & $411(4.26)$ & $442(4.58)$ & $451(4.68)$ \\
\hline$\triangle E_{\text {HOMO-LUMO }+X-}$ & $432(4.47)$ & $478(4.96)$ & $445(4.61)$ & $451(4.68)$ \\
\hline mono $/ \mathrm{kJ} / \mathrm{mol}(\mathrm{eV})$ & to $\mathrm{LUMO}+4$ & to $\mathrm{LUMO}+3$ & to $\mathrm{LUMO}+1$ & to LUMO \\
\hline$\Delta E_{S-T-m o n o} / \mathrm{kJ} / \mathrm{mol}(\mathrm{eV})$ & $154(1.60)$ & $226(2.34)$ & $262(2.71)$ & $272(2.82)$ \\
\hline $\begin{array}{l}\text { LP hybridization on } \mathrm{E} \\
\text { (monomer) }\end{array}$ & $91 \%$ s, $9 \% \mathrm{p}$ & $94 \% \mathrm{~s}, 6 \% \mathrm{p}$ & $98 \% \mathrm{~s}, 2 \% \mathrm{p}$ & $99 \% \mathrm{~s}, 1 \% \mathrm{p}$ \\
\hline $\begin{array}{l}\text { Charges }_{\text {monomer }}(\mathrm{NBO}), \\
\mathrm{E}\end{array}$ & +0.80 & +0.77 & +0.90 & +0.89 \\
\hline Charges (NBO), P & +2.06 & +2.07 & +2.06 & +2.06 \\
\hline Charges (NBO), N & -1.31 & -1.27 & -1.30 (mean) & -1.27 (mean) \\
\hline $\begin{array}{l}\text { E-E Mayer bond order } \\
\text { (dimer) }\end{array}$ & 1.03 & 0.78 & 0.41 & 0.11 \\
\hline
\end{tabular}

\begin{tabular}{|c|c|c|c|c|}
\hline \multicolumn{5}{|l|}{ pbe0/def2-tzvp ${ }^{a}$ : } \\
\hline$\Delta \varepsilon_{\text {di-mono }} / \mathrm{kJ} / \mathrm{mol}$ & -70.1 & -43.6 & -24.5 & -13.9 \\
\hline$\Delta E_{\text {di-mono }}^{0} / \mathrm{kJ} / \mathrm{mol}$ & -66.2 & -40.0 & -24.0 & -9.4 \\
\hline$\Delta H_{\text {di-mono }} / \mathrm{kJ} / \mathrm{mol}$ & -60.6 & -28.7 & -22.5 & -7.1 \\
\hline$\Delta G_{\text {di-mono }} / \mathrm{kJ} / \mathrm{mol}$ & -12.6 & -0.3 & 32.4 & 53.4 \\
\hline \multicolumn{5}{|c|}{ pbe0/def2-tzvp ${ }^{\text {a }}$ with counterpoise correction: } \\
\hline$\Delta \varepsilon_{\text {di-mono }} / \mathrm{kJ} / \mathrm{mol}$ & -56.3 & -30.1 & -15.6 & -5.3 \\
\hline$\Delta E_{\text {di-mono }}^{0} / \mathrm{kJ} / \mathrm{mol}$ & -52.4 & -26.6 & -15.0 & -0.9 \\
\hline$\Delta H_{\text {di-mono }} / \mathrm{kJ} / \mathrm{mol}$ & -46.7 & -15.3 & -13.6 & 1.4 \\
\hline$\Delta G_{\text {di-mono }} / \mathrm{kJ} / \mathrm{mol}$ & 1.2 & 13.2 & 41.3 & 62.0 \\
\hline
\end{tabular}

\begin{tabular}{lcccc}
\hline${\text { pbe0/def } 2-\text { tzvp }^{\text {a }}}^{\text {with counterpoise correction and London dispersion addition }(G D 3-B J): ~}$ & \\
$\Delta \varepsilon_{\text {di-mono }} / \mathrm{kJ} / \mathrm{mol}$ & -142.9 & -113.2 & -77.5 & -59.8 \\
$\Delta E_{\text {di-mono }}^{0} / \mathrm{kJ} / \mathrm{mol}$ & -136.9 & -106.4 & -76.0 & -58.2 \\
$\Delta H_{\text {di-mono }} / \mathrm{kJ} / \mathrm{mol}$ & -127.4 & -96.8 & -78.2 & -57.6 \\
$\Delta G_{\text {di-mono }} / \mathrm{kJ} / \mathrm{mol}$ & -87.7 & -57.4 & -3.7 & -2.7
\end{tabular}

\footnotetext{
adef2-tzvp was used for E, $\mathrm{N}$ and P. def2-svp was used for $\mathrm{C}$ and $\mathrm{H} ; \Delta E^{0}=\mathrm{ZPE}$-corrected energy, $\Delta G=$ free energy $(T=$ $298.15 \mathrm{~K}, p=1$ bar), $\Delta H=$ enthalpy. $\Delta G$ values for ${ }^{69 / 69} \mathrm{Ga}$ and ${ }^{205 / 205} \mathrm{Tl}$ given where appropriate; see the Supporting Information for full details.
} 
Using energies computed at the pbe0/def2-tzvp(E,N,P), def2-svp(C,H) level of theory (Table 2), dimer formation from two monomers via metal-metal contacts leads to significant formation enthalpies $(\Delta H)$ that diminish considerably from $\mathrm{Al}(-60.6 \mathrm{~kJ} / \mathrm{mol})$ to $\mathrm{Tl}(-7.1 \mathrm{~kJ} / \mathrm{mol})$. When the entropy $S$ is considered ( $c a .1845-1932 \mathrm{~J} / \mathrm{molK}$ for $\mathrm{Al}-\mathrm{Tl}$ ), the dimer formation from the monomers $(\Delta G)$ is expected to occur for $\mathrm{Al}(-12.6 \mathrm{~kJ} / \mathrm{mol})$, is energy neutral for $\mathrm{Ga}(-0.3 \mathrm{~kJ} / \mathrm{mol}$,), and is energetically disfavoured for In $(+32.4 \mathrm{~kJ} / \mathrm{mol})$ and $\mathrm{Tl}(+53.5 \mathrm{~kJ} / \mathrm{mol})$. The calculated Mayer bond order also decreases from an approximate single bond order (Al 1.0, Ga 0.8) to a low bond order in $\mathrm{Tl}$ (0.1); the latter can be considered a weak thallophilic interaction. ${ }^{[28]}$ Applying additional counterpoise correction raises the Gibbs energy by approximately $10 \mathrm{~kJ} / \mathrm{mol}$ for all elements and thus suggesting an energy neutral dimerization for $\mathrm{Al}$ and an increasing endoergic dimerization for the heavier elements.

It has become well-documented in recent years that London dispersion forces are a significant contributing or even dominant factor to bond strength and geometry, especially on weakly bonded molecular entities that are stabilized by sterically demanding ligands having numerous aliphatic substituents. ${ }^{[29]}$ This, together with advances in DFT methods, prompted us to include the Grimme dispersion force addition with Becke-Johnson damping (GD3-BJ) in our calculations. These show optimized geometries with significantly shorter metal-metal distances (Table 2) that appear to significantly overestimate the bonding interaction when compared to the solid state structure of $\mathbf{1}_{\mathbf{2}}$. Specifically, the Ga-Ga bond distance in the optimized molecule with dispersion forces included is $c a .3 .6 \%$ shorter than the value in the solid state of $\mathbf{1}_{2}(2.7873(12) \AA)$, compared with only $1.5 \%$ overestimation when the dispersion addition is not used. Binding enthalpies are now approximately $50 \mathrm{~kJ} / \mathrm{mol}(\mathrm{Tl})$ to $67 \mathrm{~kJ} / \mathrm{mol}$ (Al) stronger than those obtained at pbe0/def2-tzvp level of theory, and Gibbs energies are now negative for all elements, i.e. $87.7 \mathrm{~kJ} / \mathrm{mol}(\mathrm{Al})$ and $-57.4 \mathrm{~kJ} / \mathrm{mol}(\mathrm{Ga})$. One obvious reason for that is that attractive forces within a dimer are considered, i.e. intramolecular forces between two monomers that significantly affect the metal-metal bonding, but not between molecules that are important for crystal packing (intermolecular forces). Thus, these values may represent a more realistic bonding picture for one isolated dimer molecule (e.g. $\left.\mathbf{1}_{2}\right)$ in the gas phase and illustrate the influence and magnitude of London dispersion forces and other crystal packing effects on weakly bonded molecules. In accord with the shorter metal-metal bond distances, the calculated Mayer bond index on the metal-metal interaction for the $\mathrm{Al}$ and $\mathrm{Ga}$ dimers slightly increases when dispersion forces are considered.

From our experiments we know that both the gallium monomer $\mathbf{1}$ as well as the gallium dimer $\mathbf{1}_{\mathbf{2}}$ can be crystallized from benzene under identical conditions with the same lattice solvent content, and crystals of $\mathbf{1}_{2}$ dissolve in benzene to yield monomeric $\mathbf{1}$. These observations suggests that the monomer $\mathbf{1}$ and the dimer $\mathbf{1}_{2}$ are of similar energy and their interconversion appears to be 
not significally kinetically hindered either. Intermolecular forces for solid or solution phase for these species would be difficult to quantify accurately. The Grimme dispersion modified computations suggest that London dispersion forces are a significant or even dominant factor in the dimer binding energies of these molecules, with a $\Delta G^{\circ}$ of $-57.4 \mathrm{~kJ} / \mathrm{mol}$ for Ga. However, the uncorrected pbe0/def2-tzvp $(\mathrm{E}, \mathrm{N}, \mathrm{P})$, def2-svp(C,H) level of theory results in an overall not unreasonable $\Delta G$ value of $-0.3 \mathrm{~kJ} / \mathrm{mol}$ for the formation of $\mathbf{1}_{2}$ from two molecules of $\mathbf{1}$ that would support our experimental findings. This level of theory furthermore delivers a Ga-Ga bond distance that agrees better with that found from an X-ray single crystal structural characterization of $\mathbf{1}_{2}$. It should be remembered here that computations were carried out for isolated molecules in the absence of condensed phase effects. Taken as a whole, dimer formation can be regarded as weakly exoergic with a weak Ga-Ga bonding interaction that is easily perturbed. It is unclear whether the apparent better performance of the dispersion-free functional is simply due to a fortuitous cancellation of errors or some other factors, but it highlights the importance of using empirical data when validating computational methods for the study of weakly bonded species.

Even significantly shorter E-E bond lengths would be expected for the triplet state of these type of molecules as has been computationally studied for related $\beta$-diketiminate metal(I) dimers of Al-In. ${ }^{[19]}$ The singlet states for these species have been determined to being lower in energy than the triplet states, although it has been suggested that these are close in energy for aluminium. Thus, the diradicaloid triplet state may be energetically accessible in this case, and likely for a related $\left[\left({ }^{\text {Dip }} \mathrm{LAl}\right)_{2}\right]$ molecule.

The NBO analysis of the monomers and dimers shows comparable results within the series of Al-Tl with more metal-ligand mixing in the hybrid orbitals for the heavier homologues. Selected relevant orbitals for the gallium compounds are shown in Figure 5 for the monomer $\mathbf{1}$ (left) and the dimer $\mathbf{1}_{2}$ (right), and respective relevant orbitals for Al-Tl are given in the supporting information (Figures S5-S12). The HOMOs of the monomeric molecules of Al-Tl are hybrid orbitals with a significant directional metal lone pair contribution that are high in s-character with some pcontribution for the lighter metals (Table 2). For the heavier elements Ga-Tl, further significant metal lone pair contributions are found in one lower lying occupied orbital in each case (HOMO-5 for Ga, HOMO-6 for In, HOMO-10 for Tl). An unoccupied metal p-orbital perpendicular to the metal-ligand plane (LUMO+4 for Al, LUMO+3 for Ga, Fig. 5, LUMO+1 and LUMO+2 for In, LUMO for $\mathrm{Tl}$ ) compares well with those previously described for related $\beta$-diketiminate (LUMO+1) and guanidinate (LUMO) examples. The relevant HOMO to LUMO+X energy gap in the molecules reported herein, see Table 1, is for $\mathrm{Al}$ and $\mathrm{Ga}$ at the higher end compared to the range of values found for $\beta$-diketiminates ${ }^{[2 \mathrm{~d}]}$ and is significantly larger (by over 50\%) than those determined for guanidinate model complexes of $\mathrm{Al}$ and $\mathrm{Ga} \cdot{ }^{[2 \mathrm{~d}, 10 \mathrm{c}]}$ The calculated values for the 
singlet-triplet energy gaps of $\left[{ }^{\text {Dip }} \mathrm{LE}:\right], \mathrm{E}=\mathrm{Al}$, Ga, match those obtained for $\beta$-diketiminate examples, ${ }^{[2 \mathrm{~d}]}$ though their absolute numbers should be viewed with caution.

In the dimeric molecules of $\left[\left({ }^{\mathrm{Dip}} \mathrm{LE}\right)_{2}\right]$ for $\mathrm{E}=\mathrm{Al}-\mathrm{Tl}$, the HOMO contains metal lone pair contributions with higher hybrid character for the heavier homologues (see Figures S6, S8, S10, S12). For Ga (Fig. 5, right), it mainly consists of two out-of-phase gallium lone pair lobes that are non-bonding and have minor resemblance with a slipped $\pi$-bond, though with no overlap. Several hybrid orbitals contain $\sigma$-bonding interactions across the series for Al-Tl that vary in their energy content and properties. Four of these are found for the most strongly bound $\mathrm{Al}$ and $\mathrm{Ga}$ dimers. These encompass one virtual and three occupied orbitals for Al (LUMO+6, HOMO-1, HOMO-11, HOMO-15, see Figure S6) and two virtual and two occupied orbitals for Ga (LUMO+4, LUMO+2, HOMO-1, HOMO-17, see Figures 5 and S8). The unoccupied orbitals contain $\sigma$-bonding components from metal p-orbital overlap that are perpendicular to the metal-ligand plane and relate to the former LUMO+X (as given in Table 1) in the monomers. The occupied hybrid orbitals with $\sigma$-bonding interactions show a high metal s-orbital content and result from bonding combinations of the former monomer lone pairs. Two similar occupied $\sigma$-bonding orbitals are also found for the In dimer (HOMO-1, HOMO-17, Figure S10) though for the Tl-dimer, some weaker bonding contribution is found in the HOMO-21 (Figure S12). Qualitatively, $\sigma$-bonding contributions diminish for the heavier elements as expected. High lying virtual orbitals furthermore show expected $\pi$-bonding interactions, e.g. the LUMO+9 for Al (Figure S6) and Ga (Figures 5, S8), $\mathrm{LUMO}+5$ for In (Figure S10) and the LUMO+1 for $\mathrm{Tl}$ (Figure S12). $\pi^{*}$-bonding interactions (LUMO+18 for In, LUMO+12 for $\mathrm{Tl}$ ) and a $\sigma^{*}$-bonding interaction (LUMO+21 for $\mathrm{Tl}$ ) can be visualized at even higher energies. With dimer formation from the monomers, electron density appears to be withdrawn from the metal ion to the newly formed metal-metal $\sigma$-bonding interaction and could contribute to the slightly shorter metal-nitrogen bond distances (Tables 1 and 2), despite the higher metal coordination number. 

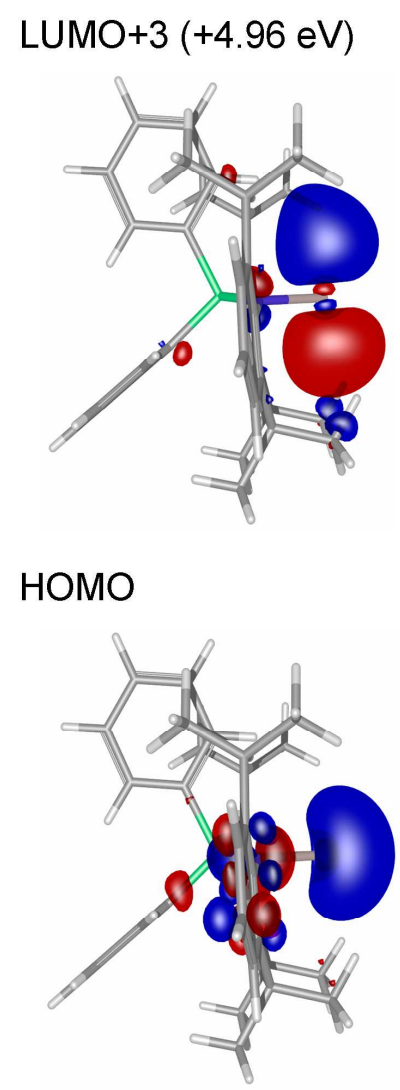

HOMO-5 (-1.59 eV)

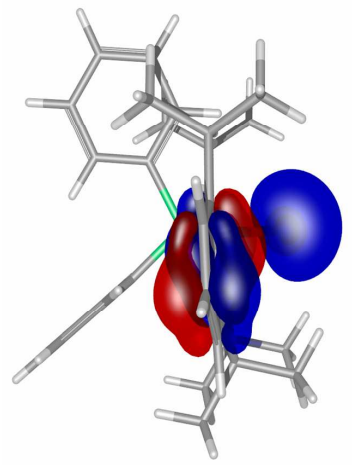

LUMO+9 (+4.47 eV)

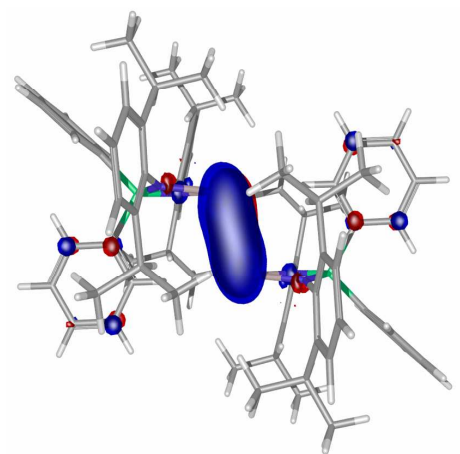

LUMO+2 (+3.58 eV)

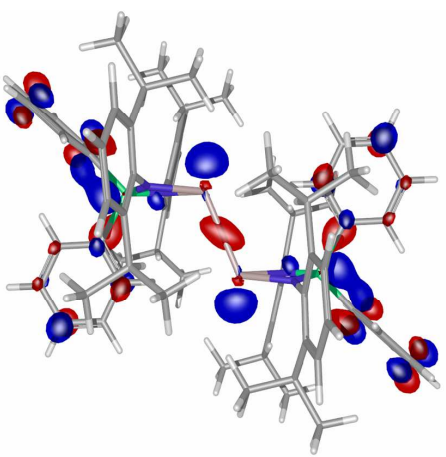

HOMO-1 (-1.30 eV)

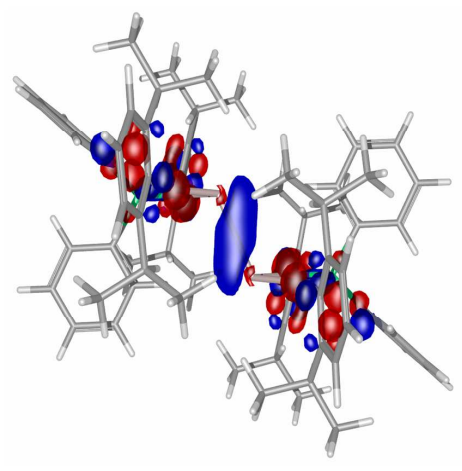

LUMO+4 (+3.79 eV)

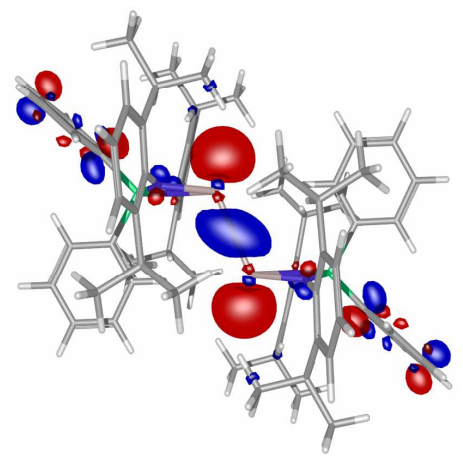

HOMO

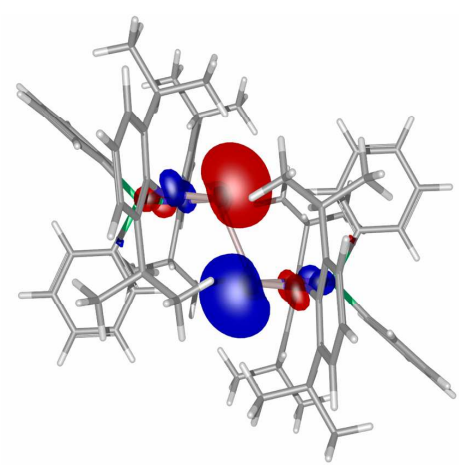

HOMO-17 (-3.03 eV)

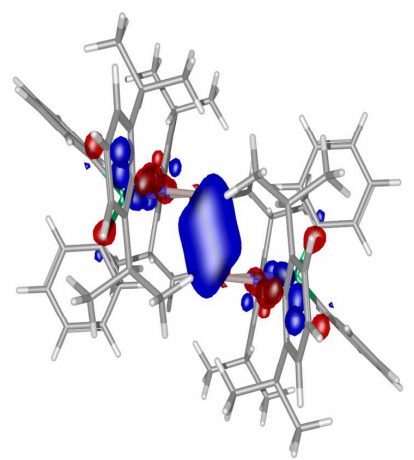

Figure 5. Selected relevant molecular orbitals of $\left[{ }^{\mathrm{Dip}} \mathrm{LGa}\right.$ :] $\mathbf{1}$ (left) and $\left[\left({ }^{\mathrm{Dip}} \mathrm{LGa}\right)_{2}\right] \mathbf{1}_{\mathbf{2}}$ (right). The orbital energies are relative to that of the respective HOMO.

Overall, the bonding description in the dimeric molecules and the relevant metal-based orbitals are similar to those found for terphenyl gallium(I) dimers, ${ }^{[21]}$ previously described weakly bonded $\beta$ diketiminate indium(I) systems and predicted for other group 13 metals. ${ }^{[7,19]}$ Heavier group 13 metal(I) dimers of the type LEEL, where $\mathrm{L}$ is a monoanionic organic substituent or ligand, have been coined "dimetallenes", to illustrate the analogy to alkenes and the possibility of an E=E double bond. ${ }^{[1,2,21]}$ The study of the bonding in these types of species has greatly shaped our understanding of multiple-bonded compounds of heavier main group elements. With respect to the trans-bent terphenyl substituted "digallenes", ArGaGaAr (where Ar is a substituted terphenyl ligand), ${ }^{[21]}$ the 
low-coordinate Ga centers may allow for some degree of multiple bonding. The bond strengths is, however, weak, as demonstrated by their dissociation into monomers and the strong influence of the ligand sterics with respect to both repulsive and attractive forces. These species are furthermore dimers of singlet state molecules, rather than dimers of triplet state molecules, and the singlet state of the dimer is preferred for the heavier group 13 metals. A similar view on multiple bonding and bond strength was discussed for the related, further reduced alkali-metal containing species $\mathrm{M}_{2}[\operatorname{ArGaGaAr}](\mathrm{M}=\mathrm{Na}, \mathrm{K})^{[21,30]}$ that have significantly shorter (by ca. $0.3 \AA$ ) Ga-Ga interactions, and have thus been named "digallynes". Bonding interactions within the molecule for the "digallynes" are once again strongly affected by the ligand sterics and the alkali metal ions. In $\mathbf{1}_{\mathbf{2}}$, the Ga-Ga bonding interaction can be simplistically described as a weak single $\sigma$-bond and no significant $\pi$-bonding interaction. Digallenes ArGaGaAr have a shorter Ga-Ga contact than that in $\mathbf{1}_{2}$ (by approximately $0.2 \AA$ ), a slightly wider C-Ga-Ga angle (ca. $123^{\circ}$ ) than the $\mathrm{P} \cdot \mathrm{G}$-Ga-Ga angle in $\mathbf{1}_{2}$ (ca. $110^{\circ}$ in the solid state, ca. $114^{\circ}$ from DFT studies), and have a lower Ga coordination number, and thus may offer a better $\pi$-bonding overlap. The unusual reactivity of terphenyl gallium(I) species is mainly facilitated by their dimers (ArGaGaAr) that can, for example, activate alkenes via lower barriers compared with their monomers because of matching frontier orbitals of correct symmetry. ${ }^{[21]}$

In addition to obvious relations to other dimeric group 13 metal(I) compounds, the set of bonding orbitals in the calculated dimer molecules $\left[\left({ }^{(\mathrm{Dip}} \mathrm{LE}\right)_{2}\right]$ compares very well with those of related group 14 element(I) species LEEL, where $\mathrm{E}=\mathrm{Si}, \mathrm{Ge}, \mathrm{Sn}$ and $\mathrm{Pb}$, and $\mathrm{L}=$ a sterically demanding amidinate or guanidinate ligand, ${ }^{[31,32]}$ albeit with a different level of orbital occupation. With $N, N^{\prime}$-chelating ligands such as amidinates and guanidinates, the group 14 element species contain E-E single-bonds and element lone pair contributions. The calculated orbitals for $\mathrm{LSiSiL}^{\text {[31a] }}$ show a $\pi$-bonding interaction in the LUMO+2 (c.f. the LUMO+9 for $\mathrm{Al}$ and $\mathrm{Ga}$ in $\left.\left[\left({ }^{\mathrm{Dip}} \mathrm{LE}\right)_{2}\right]\right)$, a $\sigma$ bonding interaction from silicon p-orbital overlap in the HOMO (c.f. the LUMO+6 for $\mathrm{Al}$ and e.g. the LUMO+4 for Ga), the out-of-phase lone pair contributions on silicon in the HOMO-1 (c.f. the HOMO for $\mathrm{Al}$ and $\mathrm{Ga}$ ) and a $\sigma$-bonding interaction from constructive silicon lone pair overlap in the HOMO-16 (c.f. the HOMO-1 and HOMO-11 for Al and HOMO-1 and HOMO-17 for Ga). Thus, the main difference is that the $\sigma$-bond from p-orbital overlap is occupied in the silicon(I) species, whereas it is unoccupied in the group 13 metal species [ $\left.\left({ }^{\mathrm{Dip}} \mathrm{LE}\right)_{2}\right]$. Accordingly and not surprisingly, the bonding between the group 13 metal(I) species has to be regarded as significantly weaker than those of related group 14 element(I) compounds and the latter complexes do not dissociate into monomeric species in aromatic hydrocarbon solutions. 


\section{Conclusions}

We have synthesized and characterized the monomeric diiminophosphinate stabilized group 13 metal(I) complexes [ $\left.{ }^{\mathrm{Dip}} \mathrm{LE}:\right](\mathrm{E}=\mathrm{Ga}$ 1, In 2, Tl 3) and obtained the solid state structure of the weakly bonded dimeric molecule $\left[\left({ }^{\mathrm{Dip}} \mathrm{LGa}\right)_{2}\right] \mathbf{1}_{2}$. Related synthetic attempts using the smaller ${ }^{\mathrm{Mes}} \mathrm{L}$ ligand afforded product mixtures from which crystals of the catenated mixed oxidation state species $\left[\left({ }^{\mathrm{Mes}} \mathrm{L}\right)_{3} \mathrm{Ga}_{4} \mathrm{I}_{3}\right] 4$ were structurally characterized, showing that sterics are an important factor in stabilizing group 13 metal(I) complexes for the lighter elements gallium and indium. The twocoordinate metal(I) centers in complexes 1-3 do not coordinate the good and small donor DMAP despite a vacant metal orbital on $\mathbf{1 - 3}$. Compound $\mathbf{1}$ is readily oxidized by dry air to form the gallium(III) oxide complex $\left[\left({ }^{\mathrm{Dip}} \mathrm{LGaO}\right)_{2}\right] \mathbf{5}$.

Complementary DFT calculations on the monomeric and dimeric molecules of $\left[{ }^{\mathrm{Dip}} \mathrm{LE}:\right](\mathrm{E}=$ Al-Tl) show that the monomers are singlet state molecules that contain a filled metal-based lone pair that is high in s-character (e.g. in the HOMO) and a vacant p-orbital (LUMO+4 to LUMO depending on group 13 metal) similar to related heterocycles. The dimer molecules contain stronger metal-metal $\sigma$-bonding interactions for the lighter group 13 elements and no significant $\pi$-bonding interaction. The HOMO shows lone pair contributions on both metal atoms, i.e. "half a lone pair" per group 13 element. Dimer formation from two monomers is exoergic for at least the lighter metals and may explains why both the monomer [ ${ }^{\mathrm{Dip}} \mathrm{LGa}$ :] $\mathbf{1}$ and the dimer [ $\left.\left({ }^{\mathrm{Dip}} \mathrm{LGa}\right)_{2}\right] \mathbf{1}_{\mathbf{2}}$ could be isolated under identical conditions. The DFT studies also support the possibility that the aluminium(I) dimer $\left[\left({ }^{\mathrm{Dip}} \mathrm{LAl}\right)_{2}\right]$, or a closely related molecule, may be prepared one day. Including a London dispersion addition to the DFT computations shows that a significant part of the binding enthalpy in the dimeric molecules (more than half for $\mathrm{Al}$ and $\mathrm{Ga}$, and the majority for $\mathrm{In}$ and $\mathrm{Tl}$ ) are contributed by dispersion forces that are predominantly a result of the sterically demanding ligands having multiple aliphatic groups.

The metal-metal bonding in the dimeric molecule $\left[\left({ }^{\mathrm{Dip}} \mathrm{LGa}\right)_{2}\right] \mathbf{1}_{2}$ could accordingly be described in a simplified form with formal charges as shown in Figure 6, i.e. as having a valence bond resonating lone pair. Thus, this bonding situation is comparable to that of alkene analogues of heavier group 14 element compounds ${ }^{[2]}$ and the orbital overlap furthermore shows similarities to dimeric group 14 element(I) compounds with $N, N^{\prime}$-chelating ligands. ${ }^{[31,32]}$ 

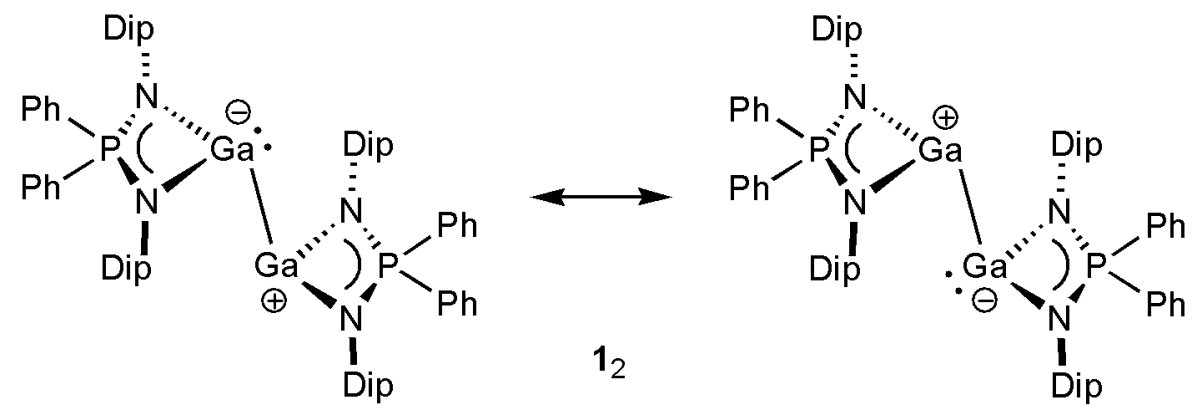

Figure 6. Chemical drawing of $\mathbf{1}_{2}$ with simplified bonding and formal charges on gallium.

\section{Experimental Section}

Full experimental details can be found in the Supporting Information.

CCDC $1503925\left(\mathbf{1}_{2} \cdot \mathrm{C}_{6} \mathrm{H}_{6}\right), 1503926\left(\mathbf{1} \cdot 0.5 \mathrm{C}_{6} \mathrm{H}_{6}\right), 1503927\left(\mathbf{2} \cdot 0.5 \mathrm{C}_{7} \mathrm{H}_{8}\right), 1503928\left(\mathbf{5} \cdot 5 \mathrm{C}_{6} \mathrm{H}_{6}\right)$ and $1503929\left(4 \cdot 0.5 \mathrm{C}_{6} \mathrm{H}_{14}\right)$, contain the supplementary crystallographic data for this paper. These data are provided free of charge by The Cambridge Crystallographic Data Centre.

\section{Acknowledgements}

A. S. is grateful to the Australian Research Council for support and a fellowship. C. A. O. thanks the Australian Research Council for Discovery Project grants DP11015530 and DP130100483. Part of this research was undertaken on the MX1 and MX2 beamlines at the Australian Synchrotron, Victoria, Australia.

Keywords: Group 13 elements $\cdot$ Low valent main group compounds $\cdot$ Metal-metal interactions $\cdot$ Nligands $\cdot \mathrm{O}-\mathrm{O}$ activation 
[1] a) W. Uhl, M. Layh, in The Group 13 Metals Aluminium, Gallium, Indium and Thallium.

Chemical Patterns and Peculiarities, ed. A. J. Downs and S. Aldridge, Wiley-Blackwell, Chichester, 2011, ch. 4, pp. 246-284; b) C. Jones, A. Stasch, in The Group 13 Metals Aluminium, Gallium, Indium and Thallium. Chemical Patterns and Peculiarities, ch. 5, pp. 285-341; c) B. F. T. Cooper, C. L. B. Macdonald, in The Group 13 Metals Aluminium, Gallium, Indium and Thallium. Chemical Patterns and Peculiarities, ch. 6, pp. 342-401; d) H. Schnöckel, A. Schnepf, in The Group 13 Metals Aluminium, Gallium, Indium and Thallium. Chemical Patterns and Peculiarities, ch. 7, pp. 402-487.

[2] Selected recent reviews: a) R. J. Wehmschulte, Top. Organomet. Chem. 2013, 41, 91-124; b) Y.-C. Tsai, Coord. Chem. Rev. 2012, 256, 722-758; c) S. Gonzalez-Gallardo, T. Bollermann, R. A. Fischer, R. Murugavel, Chem. Rev. 2012, 112, 3136-3170; d) M. Asay, C. Jones, M. Driess, Chem. Rev. 2011, 111, 354-396; e) S. Schulz, Chem. Eur. J. 2010, 16, 6416-6428; f) R. C. Fischer, P. P. Power, Chem. Rev. 2010, 110, 3877-3923; g) C. Jones, Coord. Chem. Rev. 2010, 254, 1273-1289; h) S. Gonzalez-Gallardo, G. Prabusankar, T. Cadenbach, C. Gemel, M. von Hopffgarten, G. Frenking, R. A. Fischer, Struct. Bond. 2010, 136, 147-188; i) S. Nagendran, H. W. Roesky, Organometallics 2008, 27, 457-492; j) E. Rivard, P. P. Power, Inorg. Chem. 2007, 46, 10047-10064; k) H. W. Roesky, S. S. Kumar, Chem. Commun. 2005, 4027-4038; 1) R. J. Baker, C. Jones, Coord. Chem. Rev. 2005, 249, 1857-1869; m) A. Schnepf, H. Schnöckel, Angew. Chem. Int. Ed. 2002, 41, 3532-3552; n) G. Linti, H. Schnöckel, Coord. Chem. Rev. 2000, 206-207, 285-319.

[3] C. Janiak, Coord. Chem. Rev. 1997, 163, 107-216.

[4] a) J. S. Thayer, J. Chem. Educ. 2005, 82, 1721-1727; b) D. R. McKelvey, J. Chem. Educ. 1983, $60,112-116$.

[5] a) X. Li, X. Cheng, H. Song, C. Cui, Organometallics 2007, 26, 1039-1043; b) C. Cui, H. W. Roesky, H.-G. Schmidt, M. Noltemeyer, H. Hao, F. Cimpoesu, Angew. Chem. Int. Ed. 2000, 39, 4274-4276.

[6] a) D. Dange, S. L. Choong, C. Schenk, A. Stasch, C. Jones, Dalton Trans. 2012, 41, 9304-9315;

b) S. L. Choong, W. D. Woodul, A. Stasch, C. Schenk, C. Jones, Aust. J. Chem. 2011, 64, 11731176; c) N. J. Hardman, B. E. Eichler, P. P. Power, Chem. Commun. 2000, 1991-1992.

[7] a) M. S. Hill, P. B. Hitchcock, R. Pongtavornpinyo, Dalton Trans. 2007, 731-733; b) M. S. Hill, P. B. Hitchcock, R. Pongtavornpinyo, Dalton Trans. 2005, 273-277; c) M. S. Hill, P. B. Hitchcock, R. Pongtavornpinyo, Angew. Chem. Int. Ed. 2005, 44, 4231-4235; d) M. S. Hill, P. B. Hitchcock, Chem. Commun. 2004, 1818-1819.

[8] a) Y. Liu, S. Li, X.-J. Yang, Q.-S. Li, Y. Xie, H. F. Schaefer, B. Wu, J. Organomet. Chem. 2011, 696, 1450-1455; b) I. L. Fedushkin, A. N. Lukoyanov, A. N. Tishkina, G. K. Fukin, K. A. Lyssenko, M. Hummert, Chem. Eur. J. 2010, 16, 7563-7571; c) R. J. Baker, C. Jones, D. P. Mills, 
G. A. Pierce, M. Waugh, Inorg. Chim. Acta 2008, 361, 427-435; d) I. L. Fedushkin, A. N. Lukoyanov, G. K. Fukin, S. Y. Ketkov, M. Hummert, H. Schumann, Chem. Eur. J. 2008, 14, 84658468; e) R. J. Baker, R. D. Farley,C. Jones, M. Kloth, D. M. Murphy, J. Chem. Soc., Dalton Trans. 2002, 3844-3850; f) E. S. Schmidt, A. Schier, H. Schmidbaur, J. Chem. Soc., Dalton Trans. 2001, 505-507; g) E. S. Schmidt, A. Jockisch, H. Schmidbaur, J. Am. Chem. Soc. 1999, 121, 9758-9759.

[9] M. N. Hopkinson, C. Richter, M. Schedler, F. Glorius, Nature 2014, 510, 485-496.

[10] a) J. Overgaard, C. Jones, D. Dange, J. A. Platts, Inorg. Chem. 2011, 50, 8418-8426; b) G. Jin, C. Jones, P. C. Junk, A. Stasch, W. D. Woodul, New J. Chem. 2008, 32, 835-842; c) C. Jones, P. C. Junk, J. A. Platts, A. Stasch, J. Am. Chem. Soc. 2006, 128, 2206-2207.

[11] A. Stasch, Chem. Eur. J. 2012, 18, 15105-15112.

[12] A. L. Hawley, A. Stasch, Eur. J. Inorg. Chem. 2015, 258-270.

[13] A. Stasch, Angew. Chem. Int. Ed. 2014, 53, 10200-10203.

[14] M. L. H. Green, P. Mountford, G. J. Smout, S. R. Speel, Polyhedron 1990, 9, 2763-2765.

[15] R. J. Baker, C. Jones, Dalton Trans. 2005, 1341-1348.

[16] a) X. Li, H. Song, C. Cui, Dalton Trans. 2009, 9728-9730 b) C. Jones, P. C. Junk, J. A. Platts, D. Rathmann, A. Stasch, Dalton Trans. 2005, 2497-2499.

[17] a) B. Prashanth, N. K. Srungavruksham, S. Singh, Chem. Select 2016, 1, 3601-3606; b) B. Prashanth, S. Singh, Dalton Trans. 2014, 43, 16880-16888; (b) S. A. Ahmed, M. S. Hill, P. B. Hitchcock, S. M. Mansell, O. St John, Organometallics 2007, 26, 538-549.

[18] L. Grocholl, I. Schranz, L. Stahl, Inorg. Chem. 1998, 37, 2496-2499.

[19] W. W. Schoeller, Inorg. Chem. 2011, 50, 2629-2633.

[20] M. S. Hill, P. B. Hitchcock, R. Pongtavornpinyo, Science 2006, 311, 1904-1907.

[21] a) C. A. Caputo, P. P. Power, Organometallics 2013, 32, 2278-2286; b) J. Moilanen, P. P. Power, H. M. Tuononen, Inorg. Chem. 2010, 49, 10992-11000; c) Z. Zhu, R. C. Fischer, B. D. Ellis, E. Rivard, W. A. Merrill, M. M. Olmstead, P. P. Power, J. D. Guo, S. Nagase, L. Pu, Chem. Eur. J. 2009, 15, 5263-5272; d) N. J. Hardman, R. J. Wright, A. D. Phillips, P. P. Power, J. Am. Chem. Soc. 2003, 125, 2667-2679; e) N. J. Hardman, R. J. Wright, A. D. Phillips, P. P. Power, Angew. Chem. Int. Ed. 2002, 41, 2842-2844.

[22] a) G. Linti, T. Zessin, Dalton Trans. 2011, 40, 5591-5598; b) D. Rudolf, E. Kaifer, H.-J. Himmel, Eur. J. Inorg. Chem. 2010, 4952-4961; c) C. Jones, P. C. Junk, M. Kloth, K. M. Proctor, A. Stasch, Polyhedron 2006, 25, 1592-1600.

[23] A. Seifert, G. Linti, Eur. J. Inorg. Chem. 2007, 5080-5086.

[24] N. J. Hardman, P. P. Power, Inorg. Chem. 2001, 40, 2474-2475.

[25] R. J. Baker, C. Jones, M. Kloth, Dalton Trans. 2005, 2106-2110. 
[26] M. R. Lichtenthaler, F. Stahl, D. Kratzert, B. Benkmil, H. A. Wegner, I. Krossing, Eur. J. Inorg. Chem. 2014, 4335-4341.

[27] M. C. Kuchta, J. B. Bonanno, G. Parkin, J. Am. Chem. Soc. 1996, 118, 10914-10915.

[28] P. Pyykkö, Chem. Rev. 1997, 97, 597-636.

[29] Recent examples include: a) S. Rösel, C. Balestrieri, P. R. Schreiner, Chem. Sci. 2016, in press (DOI: 10.1039/c6sc02727j); b) C. L. Wagner, L. Tao, E. J. Thompson, T. A. Stich, J. Guo, J. C. Fettinger, L. A. Berben, R. D. Britt, S. Nagase, P. P. Power, Angew. Chem. Int. Ed. 2016, 55, 10444-10447; c) J.-D. Guo, D. J. Liptrot, S. Nagase, P. P. Power, Chem. Sci. 2015, 6, 6235-6244; d) C.-Y. Lin, J. C. Fettinger, F. Grandjean, G. J. Long, P. P. Power, Inorg. Chem. 2014, 53, 9400-9406; e) C.-Y. Lin, J.-D. Guo, J. C. Fettinger, S. Nagase, F. Grandjean, G. J. Long, N. F. Chilton, P. P. Power, Inorg. Chem. 2013, 52, 13584-13593; f) S. Ndambuki, T. Ziegler, Inorg. Chem. 2012, 51, 7794-7800; g) H. Arp, J. Baumgartner, C. Marschner, P. Zark, T. Müller, J. Am. Chem. Soc. 2012, 134, 6409-6415; h) S. Grimme, P. R. Schreiner, Angew. Chem. Int. Ed. 2011, 50, 12639-12642.

[30] J. Su, X.-W. Li, R. C. Crittendon, G. H. Robinson, J. Am. Chem. Soc. 1997, 119, 5471-5472.

[31] a) C. Jones, S. J. Bonyhady, N. Holzmann, G. Frenking, A. Stasch, Inorg. Chem. 2011, 50, 12315-12325; b) S. S. Sen, A. Jana, H. W. Roesky, C. Schulzke, Angew. Chem. Int. Ed. 2009, 48, 8536-8538; c) S. Nagendran, S. S. Sen, H. W. Roesky, D. Koley, H. Grubmüller, A. Pal, R. HerbstIrmer, Organometallics 2008, 27, 5459-5463; d) S. P. Green, C. Jones, P. C. Junk, K.-A. Lippert, A. Stasch, Chem. Commun. 2006, 3978-3980.

[32] For related three-coordinate and $N, N^{\prime}$-coordinated group 14 element(I) compounds see: a) T. J. Hadlington, J. Li, M. Hermann, A. Davey, G. Frenking, C. Jones, Organometallics 2015, 34 , 3175-3185; b) S. L. Choong, C. Schenk, A. Stasch, D. Dange, C. Jones, Chem. Commun. 2012, 48, 2504-2506; c) W.-P. Leung, W.-K. Chiu, K.-H. Chong, T. C. W. Mak, Chem. Commun. 2009, $6822-6824$. 


\section{Table of contents synopsis}
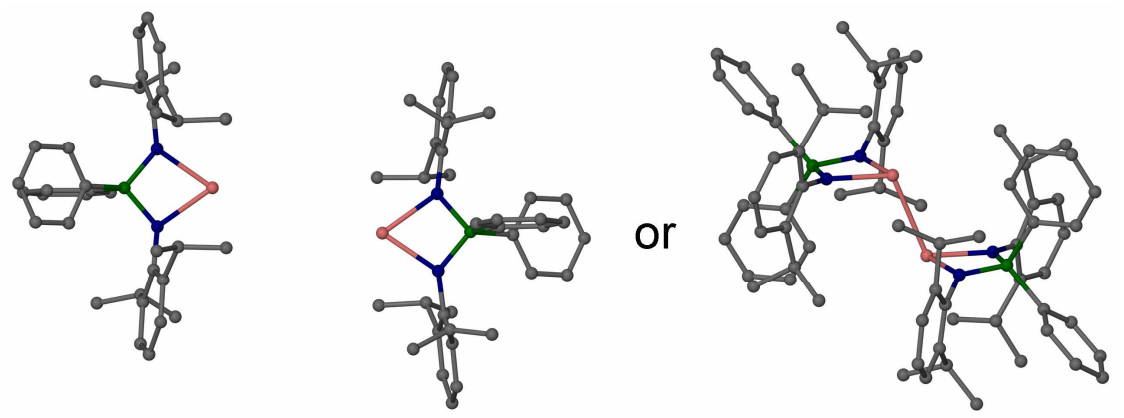

Ga goes GaGa. Sterically demanding diiminophosphinate ligands stabilize group 13 metal(I) complexes of gallium, indium and thallium. Monomeric structures with $N, N^{\prime}$-chelating ligands were found and a weakly $\sigma$-bonded dimeric structure was in addition obtained for gallium. DFT studies were conducted to investigate the nature of the metal-metal interactions. 


\section{Supporting Information (38 pages)}

\section{Table of Contents}

1.0 Experimental Section $\quad$ S2

2.0 X-ray crystallography $\quad$ S8

$\begin{array}{ll}3.0 \text { Computational studies } & \text { S11 }\end{array}$

$\begin{array}{ll}4.0 \text { References } & \text { S38 }\end{array}$ 


\subsection{Experimental Section}

\subsection{General considerations}

All manipulations were carried out using standard Schlenk and glove box techniques under an atmosphere of high purity dinitrogen. Benzene, toluene, tetrahydrofuran, $n$-hexane and cyclohexane were dried and distilled over molten potassium. ${ }^{1} \mathrm{H},{ }^{13} \mathrm{C}\left\{{ }^{1} \mathrm{H}\right\}$, and ${ }^{31} \mathrm{P}\left\{{ }^{1} \mathrm{H}\right\}$ NMR spectra were recorded on a Bruker DPX 300 or Bruker Avance 400 spectrometer in deuterated benzene and were referenced to the residual ${ }^{1} \mathrm{H}$ or ${ }^{13} \mathrm{C}\left\{{ }^{1} \mathrm{H}\right\}$ resonances of the solvent used, or external aqueous $\mathrm{H}_{3} \mathrm{PO}_{4}$ solutions, respectively. IR spectra were recorded using a Perkin Elmer RXI FT-IR spectrometer as Nujol mulls between $\mathrm{NaCl}$ plates, or on neat solids protected with a thin layer of nujol using an Agilent Cary 630 ATR FTIR spectrometer. Elemental analyses were performed by the Elemental Analysis Service at London Metropolitan University. The alkali metal complexes $\left[{ }^{\mathrm{Dip}} \mathrm{LM}\right](\mathrm{M}=\mathrm{Li}$, $\mathrm{Na}, \mathrm{K})$ were obtained as previously described. ${ }^{[\mathrm{S} 1, \mathrm{~S} 2]}$ All other reagents were used as received from commercial sources. Abbreviations: $\mathrm{br}=$ broad, $\mathrm{vbr}=$ very broad, $\mathrm{m}=$ multiplet.

[ ${ }^{\text {Dip }} \mathbf{L G a}$ (1) and [( $\left.{ }^{\text {Dip }} \mathbf{L G a}\right)_{2}$ ] $\left(\mathbf{1}_{\mathbf{2}}\right)$ : Toluene $(80 \mathrm{~mL})$ was added to a cooled $\left(-80^{\circ} \mathrm{C}\right)$ mixture of [ ${ }^{\text {Dip }} \mathrm{LNa}$ (0.82 g, $1.47 \mathrm{mmol}, 1.0$ equiv.) and "GaI" (0.36 g, $1.83 \mathrm{mmol}, 1.25$ equiv.). The reaction mixture was vigorously stirred while slowing warming to room temperature over several hours $(\mathrm{ca}$. $6 \mathrm{~h}$ ) and stirred at room temperature overnight ( $c a .16 \mathrm{~h})$. The grey-white slurry was further stirred for one hour at $40^{\circ} \mathrm{C}$ and filtered. The mixture was concentrated to $c a .5 \mathrm{~mL}, n$-hexane $(15 \mathrm{~mL})$ was added and a crystalline precipitate formed. The product was filtered off and the supernatant solution was stored at $4{ }^{\circ} \mathrm{C}$ for several days to afford a second crop. A small third crop was obtained after concentration and further storing at low temperature. All obtained crops were dried under vacuum. This procedure affords colourless $\left[{ }^{\mathrm{Dip}} \mathrm{LGa}\right](\mathbf{1})$ and/or off-white to light yellow [( $\left.\left.{ }^{\mathrm{Dip}} \mathrm{LGa}\right)_{2}\right]\left(\mathbf{1}_{\mathbf{2}}\right)$. Crystals of $\mathbf{1} \cdot 0.5 \mathrm{C}_{6} \mathrm{H}_{6}$ and $\mathbf{1}_{2} \cdot \mathrm{C}_{6} \mathrm{H}_{6}$ suitable for X-ray diffraction were obtained from a concentrated benzene solution. Crystalline material of $\left[\left({ }^{\mathrm{Dip}} \mathrm{LGa}\right)_{2}\right] \mathbf{1}_{\mathbf{2}}$ with solvent of crystallization could be obtained from $n$-hexane-rich solutions. Crystallization from cyclohexane afforded colourless tetragonal $\mathbf{1} \cdot 0.5 \mathrm{C}_{6} \mathrm{H}_{12}$. Once crystallized, the solubility of $\mathbf{1}$ and $\mathbf{1}_{2}$ can be low and product can be lost during filtration or workup. Compound $\mathbf{1}$ shows a good solubility in THF at room temperature and crystallizes in colourless form (1) as a THF-solvate at $-30^{\circ} \mathrm{C}$ and this can be used for extraction purposes. Solvates of off-white to light yellow $\mathbf{1}_{2}$ dissolve in deuterated benzene to give colourless solutions identical to those obtained for 1 according to NMR spectroscopic studies. Yield: $0.32 \mathrm{~g}$ (36\%). ${ }^{1} \mathrm{H}$ NMR $\left(\mathrm{C}_{6} \mathrm{D}_{6}, 300.1 \mathrm{MHz}, 303 \mathrm{~K}\right): \delta=1.05$ (d, $\left.J=6.9 \mathrm{~Hz}, 24 \mathrm{H}, \mathrm{CH}\left(\mathrm{CH}_{3}\right)_{2}\right), 3.84$ (sept, br, $\left.4 \mathrm{H}, \mathrm{CH}\left(\mathrm{CH}_{3}\right)_{2}\right), 6.75-7.34(\mathrm{~m}, 16 \mathrm{H}, \mathrm{Ar}-\mathrm{H}) ;{ }^{13} \mathrm{C}\left\{{ }^{1} \mathrm{H}\right\} \mathrm{NMR}\left(\mathrm{C}_{6} \mathrm{D}_{6}, 100.6 \mathrm{MHz}, 300 \mathrm{~K}\right): \delta 25.3$ $\left(\mathrm{CH}\left(\mathrm{CH}_{3}\right)_{2}\right), 29.3\left(\mathrm{CH}\left(\mathrm{CH}_{3}\right)_{2}\right), 123.3$ (br, $\left.\mathrm{ArC}\right), 124.1$ (br, $\left.\mathrm{ArC}\right), 128.0$ (ArC, partially hidden by 
strong solvent resonances), 131.3 (br, $\operatorname{ArC}$ ), 131.6 (d, not resolved, $\operatorname{ArC}$ ), $c a .135 .4$ (d, vbr, $J_{\mathrm{PC}} \approx$ $97 \mathrm{~Hz}, \operatorname{ArC}$ ), 140.2 (vbr, $\operatorname{ArC}$ ), 146.4 (br, $\operatorname{ArC}) ;{ }^{31} \mathrm{P}\left\{{ }^{1} \mathrm{H}\right\}-\mathrm{NMR}\left(\mathrm{C}_{6} \mathrm{D}_{6}, 121.5 \mathrm{MHz}, 303 \mathrm{~K}\right) \delta=16.3$ (br); ${ }^{31} \mathrm{P}\left\{{ }^{1} \mathrm{H}\right\}-\mathrm{NMR}\left(\mathrm{C}_{6} \mathrm{D}_{6}, 121.5 \mathrm{MHz}, 333 \mathrm{~K}\right) \delta=16.2$ (br), i.e. no sharpening of this resonance was observed at elevated temperatures; IR (nujol), $v \sim / \mathrm{cm}^{-1}: 1589 \mathrm{~m}, 1458 \mathrm{~s}, 1434 \mathrm{~s}, 1379 \mathrm{~s}, 1360 \mathrm{~m}$, $1312 \mathrm{~m}, 1254 \mathrm{~m}, 1235 \mathrm{~m}, 1206 \mathrm{~m}, 1186 \mathrm{~m}, 1111 \mathrm{~s}, 1043 \mathrm{~s}, 988 \mathrm{~m}, 930 \mathrm{w}, 824 \mathrm{~s}, 793 \mathrm{~m}, 777 \mathrm{~s}, 744 \mathrm{~m}$, 715m, 702s, 661m. Elemental analysis (\%) calcd. for $1, \mathrm{C}_{36} \mathrm{H}_{44} \mathrm{~N}_{2} \mathrm{GaP}: \mathrm{C}, 71.42 ; \mathrm{H}, 7.33 ; \mathrm{N}, 4.63$; found: C, 71.39; H, 7.42; N, 4.47.

[ ${ }^{\text {Dip }} \mathbf{L I n}$ ] (2): THF $(25 \mathrm{~mL})$ was added to a cooled $\left(-80^{\circ} \mathrm{C}\right)$ mixture of [ $\left.{ }^{\mathrm{Dip}} \mathrm{LNa}\right](0.58 \mathrm{~g}, 1.04 \mathrm{mmol}$, 1.0 equiv.) and finely ground $\operatorname{InBr}(0.25 \mathrm{~g}, 1.28 \mathrm{mmol}, 1.24$ equiv). The mixture was vigorously stirred while being very slowing warmed overnight $(c a .16 \mathrm{~h})$ to room temperature giving a brown and grey slurry that contained deposited indium metal. After stirring another two hours at room temperature, all volatiles were removed under vacuum. The residue was first extracted with $n$ hexane $(25 \mathrm{~mL})$, filtered and concentrated to $c a .12 \mathrm{~mL}$, and cooled to $-30^{\circ} \mathrm{C}$ to afford a crop of colourless crystals. The initial residue was further extracted with toluene $(8 \mathrm{~mL})$, filtered and concentrated to $\mathrm{ca} .5 \mathrm{~mL}$ and cooled to $4^{\circ} \mathrm{C}$ to afford a crop of colourless crystals. Both supernatant hexane and toluene solutions were further concentrated and cooled, and afforded second crops from $n$-hexane and toluene, respectively. All obtained crops were dried under vacuum. Crystals of solvates of $\mathbf{2}$ suitable for X-ray diffraction were obtained from $n$-hexane, toluene, benzene or THF, respectively. Complex $\mathbf{2}$ is the most sensitive compound in the series $\mathrm{Ga}$ to $\mathrm{Tl}$ and decomposition to form In metal can occur in solution or even the solid state. The solubility of $\mathbf{2}$ in aromatic solvents is higher than that of $\mathbf{1}$. Yield: $0.39 \mathrm{~g}(58 \%)$. Samples of the compound can decompose upon storage in the glove box at room temperature and darken with formation of indium metal. Storing the compound in a glove box freezer at $-30^{\circ} \mathrm{C}$ allowed for longer storage, although some of these samples did show signs of partial decomposition. ${ }^{1} \mathrm{H}$ NMR $\left(\mathrm{C}_{6} \mathrm{D}_{6}, 400.2 \mathrm{MHz}, 300 \mathrm{~K}\right): \delta=1.03(\mathrm{~d}$, $\left.J=6.9 \mathrm{~Hz}, 24 \mathrm{H}, \mathrm{CH}\left(\mathrm{CH}_{3}\right)_{2}\right), 3.81$ (sept, $\left.J=6.9 \mathrm{~Hz}, 4 \mathrm{H}, \mathrm{CH}\left(\mathrm{CH}_{3}\right)_{2}\right), 6.80-6.94$ (m, 6H, Ar-H), 7.03$7.28(\mathrm{~m}, 10 \mathrm{H}, \mathrm{Ar}-\mathrm{H}) ;{ }^{13} \mathrm{C}\left\{{ }^{1} \mathrm{H}\right\} \mathrm{NMR}\left(\mathrm{C}_{6} \mathrm{D}_{6}, 100.6 \mathrm{MHz}, 300 \mathrm{~K}\right): \delta 25.2\left(\mathrm{CH}\left(\mathrm{CH}_{3}\right)_{2}\right), 29.0$ $\left(C \mathrm{H}\left(\mathrm{CH}_{3}\right)_{2}\right), 123.3\left(\mathrm{~d}, J_{\mathrm{PC}}=3.1 \mathrm{~Hz}, \mathrm{ArC}\right), 123.9\left(\mathrm{~d}, J_{\mathrm{PC}}=2.1 \mathrm{~Hz}, \operatorname{ArC}\right), 128.0$ (ArC, partially hidden by strong solvent resonances), $130.5\left(\mathrm{~d}, J_{\mathrm{PC}}=2.7 \mathrm{~Hz}, \operatorname{Ar} C\right), 131.5\left(\mathrm{~d}, J_{\mathrm{PC}}=8.9 \mathrm{~Hz}, \operatorname{Ar} C\right.$ ), $137.8\left(\mathrm{~d}, J_{\mathrm{PC}}=95.0 \mathrm{~Hz}, \operatorname{Ar} C\right), 141.8(\mathrm{ArC}), 145.6\left(\mathrm{~d}, J_{\mathrm{PC}}=5.3 \mathrm{~Hz}, \operatorname{Ar} C\right) ;{ }^{31} \mathrm{P}\left\{{ }^{1} \mathrm{H}\right\}-\mathrm{NMR}\left(\mathrm{C}_{6} \mathrm{D}_{6}\right.$, $121.5 \mathrm{MHz}, 303 \mathrm{~K}) \delta=7.35$ (s, sharp); N.B. relevant NMR spectra for this compound are shown in Figures S1 and S2. IR (nujol), $v \sim / \mathrm{cm}^{-1}: 1587 \mathrm{~m}, 1461 \mathrm{~s}, 1434 \mathrm{~s}, 1378 \mathrm{~s}, 1359 \mathrm{~m}, 1332 \mathrm{~m}, 1315 \mathrm{~m}$, $1258 \mathrm{~s}, 1210 \mathrm{~m}, 1188 \mathrm{~m}, 1112 \mathrm{~s}, 1099 \mathrm{~s}, 1056 \mathrm{~m}, 1043 \mathrm{~m}, 988 \mathrm{~m}, 906 \mathrm{w}, 779 \mathrm{~s}, 753 \mathrm{~m}, 696 \mathrm{~s}, 655 \mathrm{~m}$. Values from elemental analyses (CHN combustion analysis) were repeatedly poor due to the high sensitivity of the compound and partial decomposition of sealed samples. 
[ ${ }^{\text {Dip }} \mathbf{L T I}$ (3): THF $(25 \mathrm{~mL})$ was added to a cooled $\left(-80^{\circ} \mathrm{C}\right)$ mixture of [ $\left.{ }^{\mathrm{Dip}} \mathrm{LNa}\right](0.39 \mathrm{~g}, 0.70 \mathrm{mmol}$, 1.0 equiv.) and finely powdered $\operatorname{TlBr}(0.26 \mathrm{~g}, 0.91 \mathrm{mmol}, 1.31$ equiv.). The mixture was vigorously stirred while slowing being warmed to room temperature overnight ( $c a .18 \mathrm{~h}$ ). All volatiles were removed under vacuum and the residue was first extracted with $n$-hexane $(20 \mathrm{~mL})$, filtered and concentrated to $\mathrm{ca} .8 \mathrm{~mL}$ and cooled to $-30^{\circ} \mathrm{C}$ to afford a crop of colourless crystals. The initial residue was further extracted with toluene $(10 \mathrm{~mL})$, filtered and concentrated to $c a .4 \mathrm{~mL}$ and cooled to $4^{\circ} \mathrm{C}$ to afford a crop of colourless crystals. Both supernatant hexane and toluene solutions were further concentrated and cooled, and afforded second crops from $n$-hexane and toluene, respectively. All obtained crops were dried under vacuum. X-ray diffraction experiments on crystals of various solvates of $\mathbf{3}$ repeatedly afforded datasets of poor quality with poor ordering of the molecules. Yield: $0.23 \mathrm{~g}(45 \%) ;{ }^{1} \mathrm{H}$ NMR $\left(\mathrm{C}_{6} \mathrm{D}_{6}, 300.1 \mathrm{MHz}, 303 \mathrm{~K}\right): \delta=1.03$ (d, $J=6.9 \mathrm{~Hz}, 24 \mathrm{H}$, $\left.\mathrm{CH}\left(\mathrm{CH}_{3}\right)_{2}\right), 3.83$ (sept, $\left.J=6.9 \mathrm{~Hz}, 4 \mathrm{H}, \mathrm{CH}\left(\mathrm{CH}_{3}\right)_{2}\right), 6.84-7.08$ (m, 6H, Ar-H), 7.14-7.37 (m, 10H, $\mathrm{Ar}-\mathrm{H}) ;{ }^{13} \mathrm{C}\left\{{ }^{1} \mathrm{H}\right\}$ NMR $\left(\mathrm{C}_{6} \mathrm{D}_{6}, 100.6 \mathrm{MHz}, 300 \mathrm{~K}\right): \delta 25.4-25.8\left(\mathrm{vbr}, \mathrm{CH}\left(\mathrm{CH}_{3}\right)\right), 28.5\left(\mathrm{br}, \mathrm{CH}\left(\mathrm{CH}_{3}\right)\right)$, $122.6\left(\mathrm{~d}, J_{\mathrm{PC}} \approx 2.6 \mathrm{~Hz}, \operatorname{ArC}\right), 123.5$ (d, not resolved, $\operatorname{ArC}$ ), 127.6 (ArC, partially hidden by strong solvent resonances), $129.9\left(\mathrm{~d}, J_{\mathrm{PC}}=2.7 \mathrm{~Hz}, \operatorname{ArC}\right), 131.1\left(\mathrm{~d}, J_{\mathrm{PC}}=8.5 \mathrm{~Hz}, \operatorname{ArC}\right), c a .141(\mathrm{vbr}, \operatorname{ArC})$, 142.7 (vbr, $\operatorname{ArC}$ ), 145.5 (d, not resolved, $\operatorname{ArC}$ ); ${ }^{31} \mathrm{P}\left\{{ }^{1} \mathrm{H}\right\}-\mathrm{NMR}\left(\mathrm{C}_{6} \mathrm{D}_{6}, 121.5 \mathrm{MHz}, 303 \mathrm{~K}\right) \delta=-3.7$ $\left(\mathrm{d}, \mathrm{br}, J_{205,203 \mathrm{TIP}} \approx 416 \mathrm{~Hz}\right)$. N.B.: no resonance was observed in ${ }^{205} \mathrm{Tl}$ NMR spectra over a wide range. A large chemical shift range for ${ }^{205} \mathrm{Tl}$ NMR spectroscopy, line broadening effects and splitting by the ${ }^{31} \mathrm{P}$ nucleus likely make the resonance difficult to observe. IR (nujol), $v \sim / \mathrm{cm}^{-1}$ : $1587 \mathrm{~m}, 1461 \mathrm{~s}, 1434 \mathrm{~s}, 1378 \mathrm{~s}, 1359 \mathrm{~m}, 1332 \mathrm{~m}, 1315 \mathrm{~m}, 1258 \mathrm{~s}, 1210 \mathrm{~m}, 1188 \mathrm{~m}, 1112 \mathrm{~s}, 1099 \mathrm{~s}, 1056 \mathrm{~m}$, 1043m, 988m, 906w, 779s, 753m, 696s, 655m. Elemental analysis (\%) calcd. for $\mathbf{3} 0.5 \cdot \mathrm{C}_{7} \mathrm{H}_{8}$, $\mathrm{C}_{36} \mathrm{H}_{44} \mathrm{~N}_{2} \mathrm{PTl} \cdot \mathrm{C}_{3.5} \mathrm{H}_{4}: \mathrm{C}, 60.35 ; \mathrm{H}, 6.15 ; \mathrm{N}, 3.56$; found: $\mathrm{C}, 60.29 ; \mathrm{H}, 6.12 ; \mathrm{N}, 3.59$.

$\left[\left({ }^{\text {Mes }} \mathbf{L}\right)_{3} \mathbf{G a}_{4} \mathbf{I}_{3}\right]$ 4: Few colourless rods of the title compound were obtained from an $n$-hexane extract of the reaction of [ $\left.{ }^{\mathrm{Mes}} \mathrm{LLi}\right]$ (1.0 equiv) and "GaI" (1.15 equiv) similar to the synthesis of [ ${ }^{\mathrm{Dip}} \mathrm{LGa}$ (1) that could only be structurally characterized. ${ }^{1} \mathrm{H}$ and ${ }^{31} \mathrm{P}\left\{{ }^{1} \mathrm{H}\right\}$ NMR spectroscopy showed that a mixture of many compounds had been formed.

[( $\left.\left.{ }^{\text {Dip }} \mathbf{L G a O}\right)_{2}\right]$ (5): Dry air (passed through a column of Drierite/CaSO 4 ) was blown over a stirred solution of [ $\left.{ }^{\mathrm{Dip}} \mathrm{LGa}\right](0.10 \mathrm{~g}, 0.165 \mathrm{mmol})$ in benzene $(5 \mathrm{~mL})$ for approx. 15 seconds. The solution was stirred for a further two minutes, and concentrated under vacuum to ca. $2 \mathrm{~mL}$. Storing at $6^{\circ} \mathrm{C}$ afforded colourless blocks of 5.5 $\mathrm{C}_{6} \mathrm{H}_{6}$. Yield: $c a$. $40 \mathrm{mg}(30 \%)$. A similar, parallel performed experiment in an NMR tube with J. Young valve in deuterated benzene showed a rapid reaction of 1 to $\mathbf{5}$ and some ${ }^{\text {Dip }} \mathrm{LH}$ as the main products. Once crystallized, compound $\mathbf{5}$ shows relatively low solubility in aromatic solvents. ${ }^{1} \mathrm{H}$ NMR $\left(\mathrm{C}_{6} \mathrm{D}_{6}, 300.1 \mathrm{MHz}, 303 \mathrm{~K}\right): \delta=0.78$ (vbr, 24H, 
$\left.\mathrm{CH}\left(\mathrm{CH}_{3}\right)_{2}\right), 1.39$ (vbr, 24H, $\left.\mathrm{CH}\left(\mathrm{CH}_{3}\right)_{2}\right), 3.95$ (sept, $\left.J=6.9 \mathrm{~Hz}, 8 \mathrm{H}, \mathrm{CH}\left(\mathrm{CH}_{3}\right)_{2}\right), 6.68-7.18(\mathrm{~m}, 24 \mathrm{H}$, Ar-H), 7.40-7.58 (m, 8H, Ar-H); ${ }^{31} \mathrm{P}\left\{{ }^{1} \mathrm{H}\right\}-\mathrm{NMR}\left(\mathrm{C}_{6} \mathrm{D}_{6}, 121.5 \mathrm{MHz}, 303 \mathrm{~K}\right) \delta=17.5$ (s, sharp); N.B.: The two very broad methyl resonances coalesce at $c a \cdot 55-60^{\circ} \mathrm{C}$ to one very broad resonance. Spectral data at $70^{\circ} \mathrm{C}:{ }^{1} \mathrm{H}$ NMR $\left(\mathrm{C}_{6} \mathrm{D}_{6}, 300.1 \mathrm{MHz}, 343 \mathrm{~K}\right): \delta=c a .1 .1$ (vbr, $\left.48 \mathrm{H}, \mathrm{CH}\left(\mathrm{CH}_{3}\right)_{2}\right), 3.92$ (sept, $\left.J=6.9 \mathrm{~Hz}, 8 \mathrm{H}, \mathrm{CH}\left(\mathrm{CH}_{3}\right)_{2}\right), 6.78-7.15(\mathrm{~m}, 24 \mathrm{H}, \operatorname{Ar}-\mathrm{H}), 7.48-7.57$ (m, 8H, Ar-H); ${ }^{13} \mathrm{C}\left\{{ }^{1} \mathrm{H}\right\} \mathrm{NMR}\left(\mathrm{C}_{6} \mathrm{D}_{6}, 75.5 \mathrm{MHz}, 343 \mathrm{~K}\right): \delta 24.2\left(\mathrm{vbr}, \mathrm{CH}\left(\mathrm{CH}_{3}\right)_{2}\right), 28.9\left(\mathrm{CH}\left(\mathrm{CH}_{3}\right)_{2}\right), 123.7\left(\mathrm{~d}, J_{\mathrm{PC}}=\right.$ $2.0 \mathrm{~Hz}, \operatorname{ArC}), 125.3\left(\mathrm{~d}, J_{\mathrm{PC}}=2.6 \mathrm{~Hz}, \operatorname{ArC}\right), 127.6$ (ArC, partially hidden by strong solvent resonances), $131.4\left(\mathrm{~d}, J_{\mathrm{PC}}=2.9 \mathrm{~Hz}, \operatorname{ArC}\right), 132.4\left(\mathrm{~d}, J_{\mathrm{PC}}=9.2 \mathrm{~Hz}, \operatorname{ArC}\right), 132.5\left(\mathrm{~d}, J_{\mathrm{PC}}=99.9 \mathrm{~Hz}\right.$, $\operatorname{ArC}$ ), $136.9\left(\mathrm{~d}, J_{\mathrm{PC}}=1.9 \mathrm{~Hz}, \operatorname{ArC}\right), 147.9\left(\mathrm{~d}, J_{\mathrm{PC}}=4.7 \mathrm{~Hz}, \operatorname{ArC}\right.$ ); IR (nujol), v / $/ \mathrm{cm}^{-1}: 1589 \mathrm{~m}$, 1463s, 1435s, 1385m, 1365s, 1318m, 1256s, 1241m, 1206s, 1119s, 1104s, 1042m, 989s, 934m, 820m, 792s, 754m,707m, 698s, 635s. Elemental analysis (\%) calcd. for 5 (vacuum dried sample), $\mathrm{C}_{72} \mathrm{H}_{88} \mathrm{~N}_{4} \mathrm{P}_{2} \mathrm{Ga}_{2} \mathrm{O}_{2}$ : C, 69.58; H, 7.14; N, 4.51; found: C, 69.83, H, 7.09; N, 4.36.

\subsection{NMR Spectroscopy}

Because of the sensitive nature of complex [ ${ }^{\text {Dip }}$ LIn:] 2, no elemental (combustion) analysis of suitable quality was obtained and relevant NMR spectra of this compound are shown below (Figures S1 and S2) as representative examples of this compound class. NMR spectra were acquired in $5 \mathrm{~mm}$ NMR tubes with J.Young stopcock using appropriately dried and degassed deuterated solvents. 

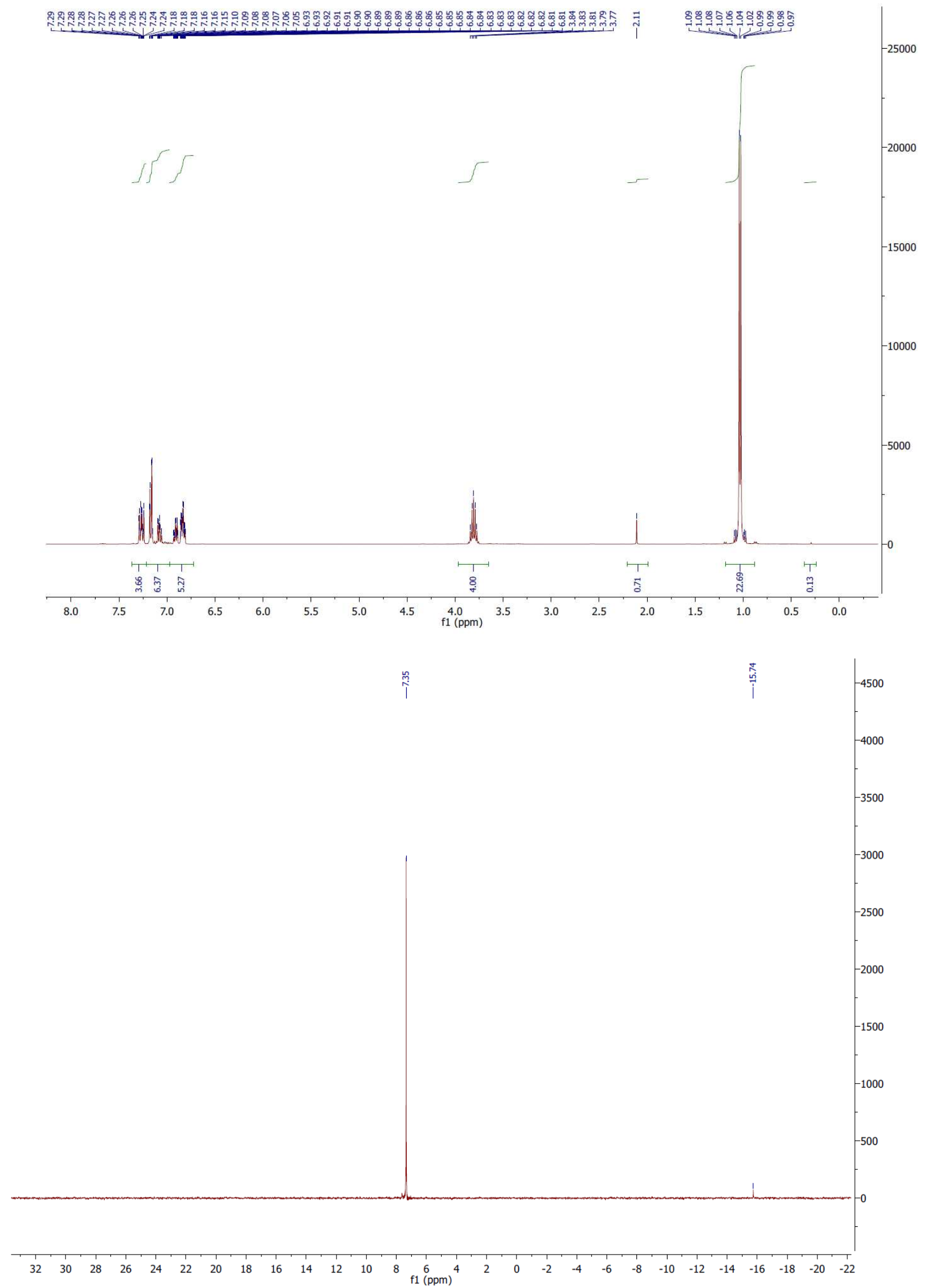

Figure S1. ${ }^{1} \mathrm{H}$ (400 MHz), top, and ${ }^{31} \mathrm{P}\left\{{ }^{1} \mathrm{H}\right\}$ (121.5 MHz), bottom, NMR spectra of [ ${ }^{\text {Dip }}$ LIn:] 2 in deuterated benzene. The minor resonances at $\delta 2.11\left({ }^{1} \mathrm{H}\right)$ shows (former) lattice toluene, ${ }^{[\mathrm{S} 3]}$ and ${ }^{\text {Dip }} \mathrm{LH}$ at $\delta-15.7 \mathrm{ppm}\left({ }^{31} \mathrm{P}\left\{{ }^{1} \mathrm{H}\right\}\right)$ from minor decomposition during sample preparation. 


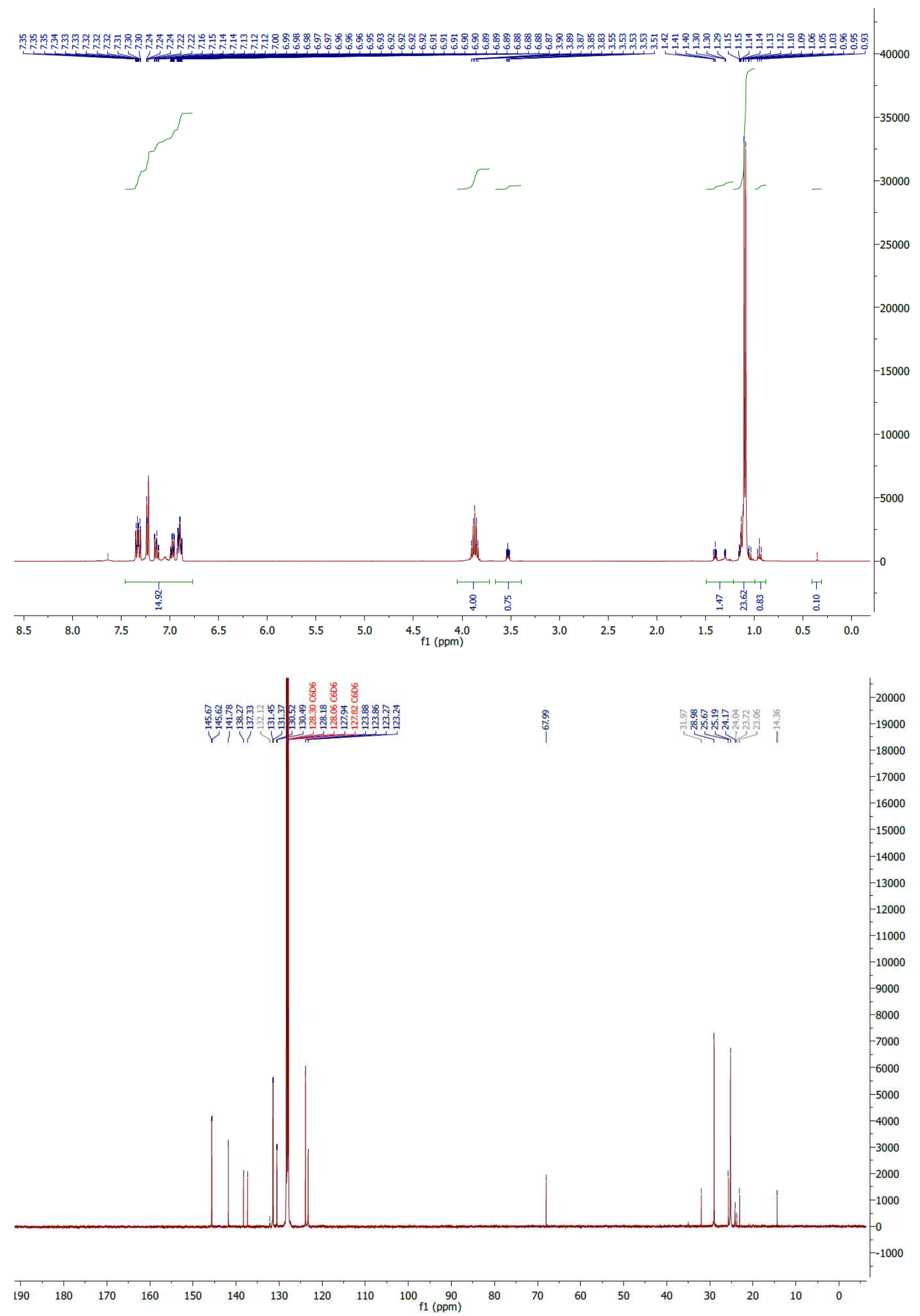

Figure S2. ${ }^{1} \mathrm{H}(400 \mathrm{MHz})$, top, and ${ }^{13} \mathrm{C}\left\{{ }^{1} \mathrm{H}\right\}(100.6 \mathrm{MHz})$, bottom, NMR spectra of [ ${ }^{\mathrm{Dip}}$ LIn:] 2 in deuterated benzene, including small quantities of THF and $n$-hexane. ${ }^{[\mathrm{S} 3]}$ 


\subsection{X-ray crystallography}

Suitable crystals were mounted in silicone oil and were either measured using an Oxford Xcalibur Gemini Ultra $\left(\mathbf{2} \cdot 0.5 \mathrm{C}_{7} \mathrm{H}_{8}\right)$ or a Bruker X8 Apex II CCD $\left(\mathbf{5} \cdot 5 \mathrm{C}_{6} \mathrm{H}_{6}\right)$ diffractometer with $\mathrm{Mo}_{\mathrm{K} \alpha}$ radiation $(\lambda=0.71073 \AA$ ), or at the MX1 beamline at the Australian Synchrotron (all others) using synchrotron radiation with a wavelength close to $\mathrm{Mo}_{\mathrm{K} \alpha}$ radiation. Data collection at the synchrotron was performed using the Blu-Ice software package, ${ }^{[\mathrm{S} 4]}$ and data reduction was performed using XDS. ${ }^{\left[{ }^{[5]}\right.}$ All structures were refined using SHELX, ${ }^{[\mathrm{S} 6]}$ and all non-hydrogen atoms were refined anisotropically.

The compounds [ $\left.{ }^{\mathrm{Dip}} \mathrm{LE}:\right](\mathrm{E}=\mathrm{Ga}$ 1, In 2, Tl 3) predominantly crystallize with solvent of crystallization (e.g. $n$-hexane, benzene, toluene, THF) in colourless form in the tetragonal crystal system (cell dimensions ca. 25.7, 25.7, 10.7, 90, 90, 90). The overall packing of the main molecules appeared to be not significantly affected by the type of lattice solvent. The lattice solvent was severely disordered in the respective crystal structures, and its type could be confirmed by NMR spectroscopy. For a partial packing of the molecules, see Figure S3. For E = Ga, the yellow dimer $\mathbf{1}_{2}$ could be obtained from benzene as well. All datasets acquired for $\mathrm{E}=\mathrm{Tl}$ refined poorly and suggest poor overall ordering, though the connectivity was unambiguously established. Some data collections for samples of [ ${ }^{\text {Dip }}$ LE:] E = In 2 and Tl 3 were indexed in tetragonal cells (of ca. 25.6, 25.6, 31.9, 90, 90, 90), and refinement attempts indicate deviation from perfect ordering and refined poorly.

The crystal structures of $\mathbf{1} \cdot 0.5 \mathrm{C}_{6} \mathrm{H}_{6}$ and $\mathbf{2} \cdot 0.5 \mathrm{C}_{7} \mathrm{H}_{8}$ are isomorphous and crystallize with half a molecule in the asymmetric unit. One isopropyl group (C17-C19) is disordered and was refined with two positions for each atom using geometry restraints. The severely disordered solvent of crystallization was in both cases removed using the PLATON/SQUEEZE routine, ${ }^{[\mathrm{S} 7]}$ see the cif file for further details. During the refinement of the crystal structure of $\mathbf{1}_{2} \cdot \mathrm{C}_{6} \mathrm{H}_{6}$, geometry restraints were applied to the lattice solvent molecule.

In the crystal structure of $\mathbf{4} \cdot 0.5 \mathrm{C}_{6} \mathrm{H}_{14}$ geometry restraints were applied during the refinement to few of the ligand aryl groups because of the large libration modes of these groups. Severely disordered solvent of crystallization, $n$-hexane, was removed using the PLATON/SQUEEZE routine, ${ }^{[S 4]}$ see the cif file for further details.

Complex $5.5 \mathrm{C}_{6} \mathrm{H}_{6}$, crystallized with half a molecule of $\mathbf{5}$ in the asymmetric unit. The asymmetric unit contained one full molecule of benzene that was refined using geometry restraints, one full molecule of benzene that was disordered and modelled with positions for each atom and refined using geometry restraints. A further half molecule of benzene per asymmetric unit was severely 
disordered and was removed using the PLATON/SQUEEZE routine, ${ }^{[S 4]}$ see the cif file for further details.

Selected bond lengths and angles of all crystal structures are collected in the main text in Table 1 or the Figure captions. Refinement details are summarized in Table S1. CCDC 1503925 - 1503929 contains the supplementary crystallographic data for this paper. These data can be obtained free of charge from The Cambridge Crystallographic Data Centre via www.ccdc.cam.ac.uk/data_request/cif.

Table S1 Crystallographic data.

\begin{tabular}{|c|c|c|c|c|c|}
\hline Compound reference & $\mathbf{1} \cdot 0.5 \mathrm{C}_{6} \mathrm{H}_{6}$ & $2 \cdot 0.5 \mathrm{C}_{7} \mathrm{H}_{8}$ & $\mathbf{1}_{2} \cdot \mathrm{C}_{6} \mathrm{H}_{6}$ & $4 \cdot 0.5 \mathrm{C}_{6} \mathrm{H}_{14}$ & $5 \cdot 5 \mathrm{C}_{6} \mathrm{H}_{6}$ \\
\hline Chemical formula & $\mathrm{C}_{39} \mathrm{H}_{47} \mathrm{GaN}_{2} \mathrm{P}$ & $\mathrm{C}_{39.50} \mathrm{H}_{48} \mathrm{InN}_{2}$ & $\mathrm{C}_{78} \mathrm{H}_{94} \mathrm{Ga}_{2} \mathrm{~N}_{4} \mathrm{P}_{2}$ & $\mathrm{C}_{93} \mathrm{H}_{103} \mathrm{Ga}_{4} \mathrm{I}_{3} \mathrm{~N}_{6} \mathrm{P}_{3}$ & $\mathrm{C}_{102} \mathrm{H}_{118} \mathrm{Ga}_{2} \mathrm{~N}_{4} \mathrm{O}_{2} \mathrm{P}_{2}$ \\
\hline Formula Mass & 644.47 & 696.59 & 1288.95 & 2057.30 & 1633.38 \\
\hline Crystal system & Tetragonal & Tetragonal & Monoclinic & Orthorhombic & Triclinic \\
\hline$a / \AA$ & $25.658(4)$ & $25.796(4)$ & $23.603(5)$ & $15.118(3)$ & $12.0320(5)$ \\
\hline$b / \AA$ & $25.658(4)$ & $25.796(4)$ & $13.111(3)$ & $27.674(6)$ & $12.7090(5)$ \\
\hline$c / \AA$ & $10.622(2)$ & $10.629(2)$ & $23.710(5)$ & $27.917(6)$ & $15.2478(6)$ \\
\hline$\alpha /^{\circ}$ & 90 & 90 & 90 & 90 & $89.085(2)$ \\
\hline$\beta 1^{\circ}$ & 90 & 90 & $108.16(3)$ & 90 & $83.106(2)$ \\
\hline$\gamma /{ }^{\circ}$ & 90 & 90 & 90 & 90 & $70.070(2)$ \\
\hline Unit cell volume $/ \AA^{3}$ & 6993(2) & $7073(2)$ & 6972(3) & $11680(4)$ & $2175.35(15)$ \\
\hline Temperature/K & $100(2)$ & $123(2)$ & $100(2)$ & $100(2)$ & $123(2)$ \\
\hline Space group & $I 4_{1} m d$ & $I 4_{1} m d$ & $C 2 / c$ & Pnma & $P 1$ \\
\hline $\begin{array}{l}\text { No. of formula units per unit } \\
\text { cell, } Z\end{array}$ & 8 & 8 & 4 & 4 & 2 \\
\hline Radiation type & Synchrotron $\alpha$ & $\mathrm{Mo}_{\mathrm{K} \alpha}$ & Synchrotron & Synchrotron & $\mathrm{Mo}_{\mathrm{K} \alpha}$ \\
\hline Wavelength/Å & 0.7108 & 0.71073 & 0.7108 & 0.7109 & 0.71073 \\
\hline Density (calc) $/ \mathrm{Mg} / \mathrm{m}^{3}$ & 1.224 & 1.308 & 1.228 & $1.170 \mathrm{Mg} / \mathrm{m} 3$ & 1.247 \\
\hline $\begin{array}{l}\text { Absorption coefficient, } \\
\mu / \mathrm{mm}^{-1}\end{array}$ & 0.861 & 0.742 & 0.864 & 1.783 & 0.708 \\
\hline$F(000)$ & 2728 & 2904 & 2728 & 4124 & 866 \\
\hline Reflections collected & 31205 & 35023 & 30867 & 144464 & 63308 \\
\hline Independent reflections & 4340 & 5395 & 8224 & 14026 & 17530 \\
\hline Theta range $/^{\circ}$ & 1.587 to 27.869 & 2.072 to 30.800 & 1.816 to 27.923 & 1.532 to 27.829 & 1.705 to 34.160 \\
\hline Completeness (to theta) $/ \%$ & $99.6\left(\right.$ at $25.24^{\circ}$ ) & $99.8\left(\right.$ at $\left.25.24^{\circ}\right)$ & $99.3\left(\right.$ at $25.24^{\circ}$ ) & $99.6\left(\right.$ at $\left.25.25^{\circ}\right)$ & $99.7\left(\right.$ at $25.24^{\circ}$ ) \\
\hline$R_{\text {int }}$ & 0.0664 & 0.0311 & 0.0829 & 0.0715 & 0.0330 \\
\hline Data / restraints / parameter & $4340 / 13 / 231$ & $5395 / 31 / 224$ & 8224 / 2 / 397 & $14026 / 18 / 508$ & $17530 / 36 / 541$ \\
\hline Final $R_{I}$ values $(I>2 \sigma(I))$ & 0.0497 & 0.0294 & 0.0455 & 0.0634 & 0.0361 \\
\hline $\begin{array}{l}\text { Final } w R\left(F^{2}\right) \text { values }(I> \\
2 \sigma(I))\end{array}$ & 0.1293 & 0.0694 & 0.1018 & 0.1654 & 0.0965 \\
\hline Final $R_{l}$ values (all data) & 0.0547 & 0.0321 & 0.0780 & 0.0834 & 0.0472 \\
\hline Final $w R\left(F^{2}\right)$ values (all data) & 0.1328 & 0.0709 & 0.1152 & 0.1763 & 0.1008 \\
\hline Goodness of fit on $F^{2}$ & 1.043 & 1.075 & 1.008 & 1.092 & 1.084 \\
\hline $\begin{array}{l}\text { Largest diff. peak and } \\
\text { hole/e } \cdot \AA^{-3}\end{array}$ & $0.416,-0.701$ & $0.307,-0.400$ & $0.689,-0.770$ & $2.409,-1.406$ & $0.995,-0.358$ \\
\hline Absolute structure parameter & $0.027(7)$ & $-0.020(8)$ & - & - & - \\
\hline CCDC number & 1503926 & 1503927 & 1503925 & 1503929 & 1503928 \\
\hline
\end{tabular}



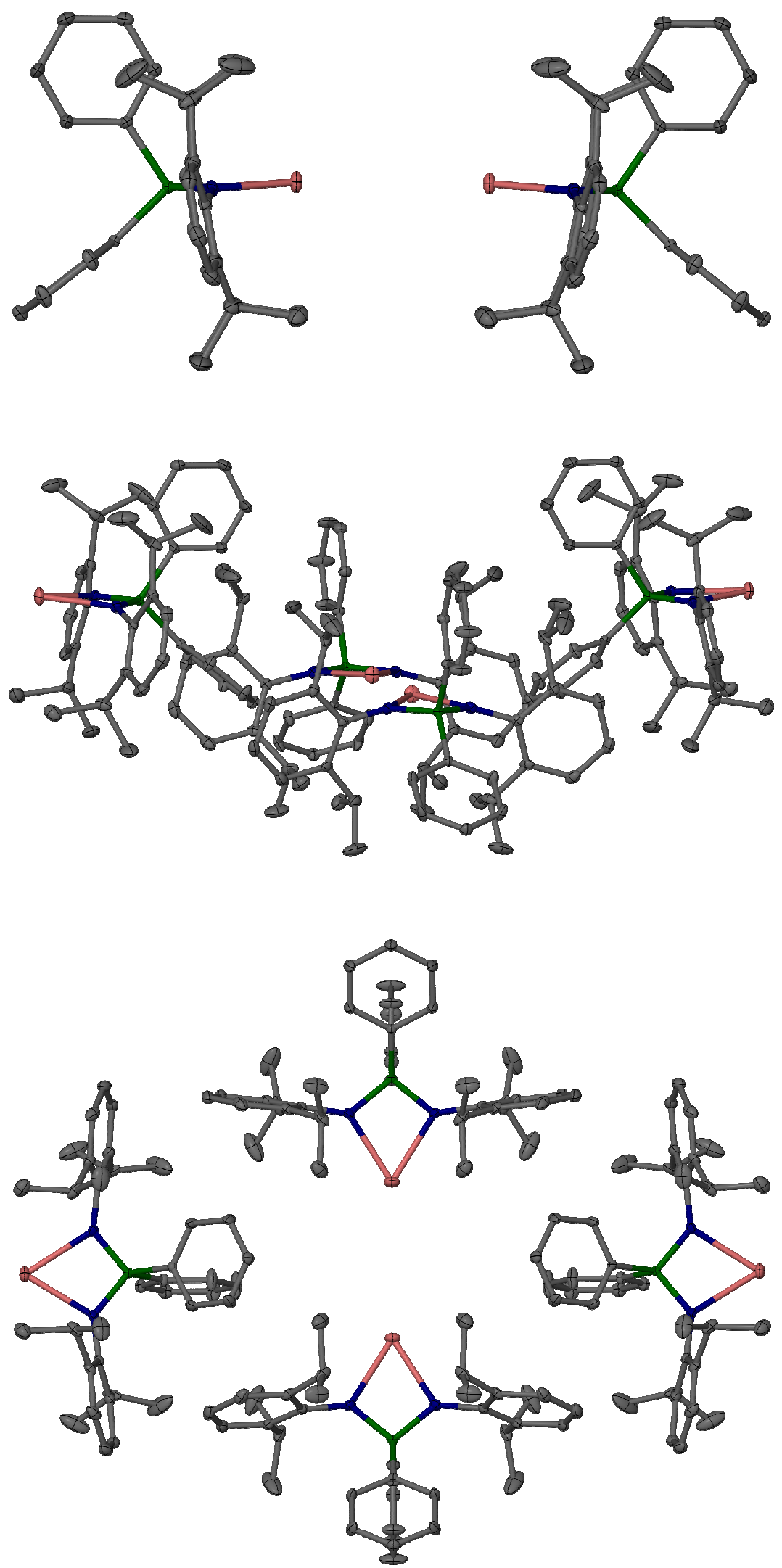

Figure S3. Partial packing of [ ${ }^{\mathrm{Dip}} \mathrm{LIn}$ :] 2 in the tetragonal crystal system (In: salmon, P: green, N: dark blue, C: gray). Top: two nearest neighbours; middle and bottom: four nearest molecules in different views. The voids between the molecules are partially filled with severely disordered solvent molecules. Minor disordered parts of isopropyl groups, hydrogen atoms and disordered solvent omitted for clarity. 


\subsection{Computational studies}

Computations using density functional theory were carried out at the $\mathrm{pbe} 0^{[\mathrm{S} 8]} / \mathrm{def} 2$-svp ${ }^{[\mathrm{S} 9]}$, pbe0/def2-tzvp (E,N,P), def2-svp (C,H) and pbe0-GD3-BJ ${ }^{[\mathrm{S} 10]} / \mathrm{def} 2-\operatorname{tzvp}(\mathrm{E}, \mathrm{N}, \mathrm{P})$, def2-svp (C,H) levels of theory using G09 D.01. ${ }^{[\mathrm{S} 11]}$ Basis sets were obtained from the Basis Set Exchange. ${ }^{[\mathrm{S} 12]}$ All computations were carried out on the full monomers $\left[{ }^{\mathrm{Dip}} \mathrm{LE}:\right](\mathrm{E}=\mathrm{Al}, \mathrm{Ga}, \mathrm{In}, \mathrm{Tl})$ and the respective dimers $\left[\left({ }^{\mathrm{Dip}} \mathrm{LE}\right)_{2}\right]$. The monomers and dimers were optimized in their singlet states; the monomeric molecules were also optimized in their triplet states. Natural bond order analyses were carried out using NBO6. ${ }^{[\mathrm{S} 13]}$

Section 3.1 gives relevant data for the calculates structures, section 3.2 shows relevant orbitals and section 3.3 contains Cartesian coordinates of the optimized structures.
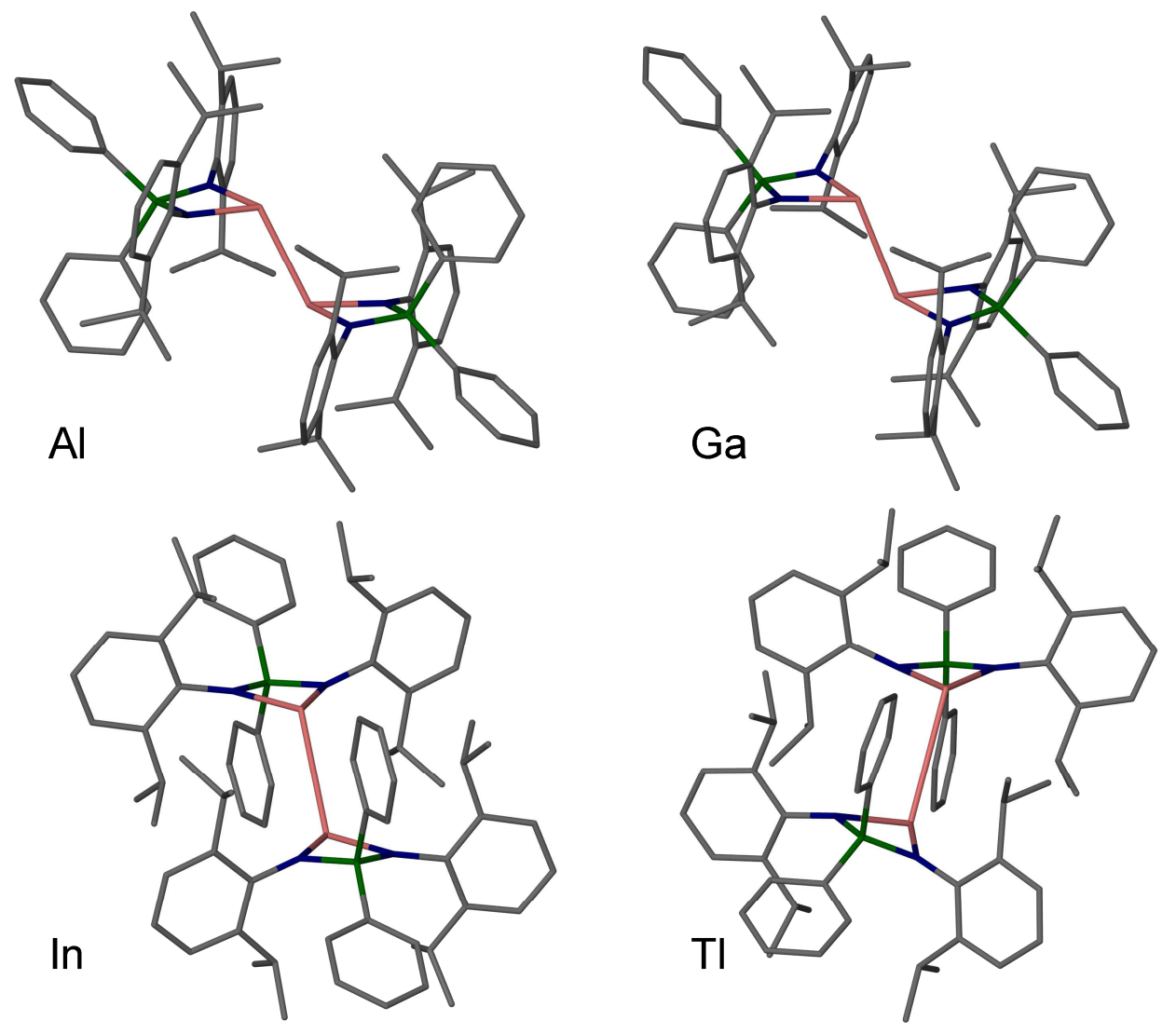

Figure S4. Optimized DFT (pbe0/def2-tzvp) geometries for the dimers $\left[\left({ }^{\mathrm{Dip}} \mathrm{LE}\right)_{2}\right], \mathrm{E}=\mathrm{Al}, \mathrm{Ga}, \mathrm{In}$, Tl. 
3.1 Tabulated data for $\left[{ }^{\mathrm{Dip}} \mathrm{LE}:\right]$ and $\left[\left({ }^{\mathrm{Dip}} \mathrm{LE}\right)_{2}\right](\mathrm{E}=\mathrm{Al}, \mathrm{Ga}, \mathrm{In}, \mathrm{Tl})$ at different levels of theory

Table S2. Calculated energies for the monomers [ $\left.{ }^{\text {Dip }} \mathrm{LE}:\right](\mathrm{E}=\mathrm{Al}, \mathrm{Ga}, \mathrm{In}, \mathrm{Tl})$.

\begin{tabular}{|c|c|c|c|c|c|}
\hline $\mathbf{E}=$ & Theory level & $\varepsilon$ (a.u.) & $\mathrm{ZPE}_{\text {corr }}$ (a.u.) & $\mathbf{H}_{\text {corr }}$ (a.u.) & $\mathbf{S}(\mathrm{J} / \mathrm{mol} \cdot \mathbf{K})$ \\
\hline \multirow[t]{3}{*}{$\mathrm{Al}^{27}$} & pbe0/def2-svp & -2088.39541825 & 0.717465 & 0.758811 & 1012.2 \\
\hline & pbe0/def2-tzvp ${ }^{a}$ & -2088.73132006 & 0.717449 & 0.758651 & 1002.9 \\
\hline & pbe0-GD3-BJ/def2-tzvpa & -2088.86631735 & 0.718369 & 0.758444 & 965.81 \\
\hline \multirow[t]{3}{*}{$\mathrm{Ga}^{69}$} & pbe0/def2-svp & -3770.51439890 & 0.716609 & 0.757381 & 988.41 \\
\hline & pbe0/def2-tzvp ${ }^{a}$ & -3771.01250143 & 0.716525 & 0.757321 & 989.60 \\
\hline & pbe0-GD3-BJ/def2-tzvpa & -3771.14786513 & 0.717347 & 0.757959 & 979.90 \\
\hline \multirow[t]{3}{*}{$\operatorname{In}^{115}$} & pbe0/def2-svp & -2036.30052368 & 0.716120 & 0.758871 & 1058.7 \\
\hline & pbe0/def2-tzvp ${ }^{a}$ & -2036.55840971 & 0.716049 & 0.758748 & 1048.4 \\
\hline & pbe0-GD3-BJ/def2-tzvpa & -2036.69653179 & 0.717165 & 0.759640 & 1037.5 \\
\hline \multirow[t]{3}{*}{$\mathrm{Tl}^{205}$} & pbe0/def2-svp & -2018.67531333 & 0.715763 & 0.758601 & 1057.8 \\
\hline & pbe0/def2-tzvp ${ }^{a}$ & -2018.93204173 & 0.715376 & 0.758365 & 1067.6 \\
\hline & pbe0-GD3-BJ/def2-tzvpa & -2019.07148099 & 0.716738 & 0.759338 & 1044.6 \\
\hline
\end{tabular}

${ }^{a}$ def2-svp for C, H. def2-tzvp for all other elements.

Table S3. Energies of structurally optimised singlet and triplet states of the monomers [ $\left.{ }^{\text {Dip }} \mathrm{LE}:\right](\mathrm{E}=$ $\mathrm{Al}, \mathrm{Ga}, \mathrm{In}, \mathrm{Tl})$. All energies calculated using OPBE/def2-svp on geometries optimised at PBE0/def2-svp. $\Delta \varepsilon$ is relative to the singlet state.

\begin{tabular}{llll}
\hline $\mathbf{E}=$ & Singlet $(\varepsilon$, a.u. $)$ & Triplet $(\varepsilon$, a.u. $)$ & $\Delta \varepsilon(\mathbf{k J})$ \\
\hline $\mathrm{Al}$ & -2089.69794061 & -2089.63476108 & 165.88 \\
$\mathrm{Ga}$ & -3772.54438908 & -3772.45647365 & 230.82 \\
$\mathrm{In}$ & -2037.86606540 & -2037.76492508 & 265.54 \\
$\mathrm{Tl}$ & -2020.24485380 & -2020.14074750 & 273.33 \\
\hline
\end{tabular}

Table S4. Energies of singlet and triplet states of the monomers $\left[{ }^{\mathrm{Dip}} \mathrm{LE}:\right](\mathrm{E}=\mathrm{Al}, \mathrm{Ga}, \mathrm{In}, \mathrm{Tl}) . \mathrm{All}$ energies calculated using OPBE/def2-tzvp $(\mathrm{E}, \mathrm{N}, \mathrm{P})$, def2-svp $(\mathrm{C}, \mathrm{H})$ on geometries optimised at PBE0/def2-tzvp $(\mathrm{E}, \mathrm{N}, \mathrm{P}), \operatorname{def} 2-\operatorname{svp}(\mathrm{C}, \mathrm{H}) . \Delta \varepsilon$ is relative to the singlet state.

\begin{tabular}{llll}
\hline $\mathbf{E}=$ & Singlet $(\boldsymbol{\varepsilon}$, a.u. $)$ & Triplet $(\boldsymbol{\varepsilon}$, a.u. $)$ & $\Delta \varepsilon(\mathbf{k J})$ \\
\hline $\mathrm{Al}$ & -2090.02935745 & -2089.97054616 & 154.41 \\
$\mathrm{Ga}$ & -3773.03687883 & -3772.95077972 & 226.05 \\
$\mathrm{In}$ & -2038.11976492 & -2038.02001185 & 261.90 \\
$\mathrm{Tl}$ & -2020.49837176 & -2020.39462914 & 272.38 \\
\hline
\end{tabular}

Table S5. E-E distances $(\AA)$ in the dimer complexes $\left[\left({ }^{\mathrm{Dip}} \mathrm{LE}\right)_{2}\right]$ at different levels of theory Theory level

\begin{tabular}{lrrr}
$\mathrm{E}=$ & pbe0/def2-svp & pbe0/def2-tzvp $^{\mathrm{a}}$ & pbe0-GD3-BJ/def2-tzvp $^{\mathrm{a}}$ \\
\hline $\mathrm{Al}$ & 2.766 & 2.762 & 2.679 \\
$\mathrm{Ga}$ & 2.822 & 2.830 & 2.689 \\
$\mathrm{In}$ & 3.318 & 3.289 & 3.083 \\
$\mathrm{Tl}$ & 3.694 & 3.688 & 3.275 \\
\hline
\end{tabular}

${ }^{a}$ def2-svp for C, H. def2-tzvp for all other elements. 
Table S6. P...E-E angles $\left({ }^{\circ}\right)$ in the dimer complexes $\left[\left({ }^{\text {Dip }} \mathrm{LE}\right)_{2}\right]$ at different levels of theory Theory level

\begin{tabular}{lrr}
$\mathrm{E}=$ & pbe0/def2-svp & pbe0/def2-tzvp $^{\mathrm{a}}$ \\
\hline $\mathrm{Al}$ & 117.469 & 117.535 \\
$\mathrm{Ga}$ & 113.457 & 114.062 \\
$\mathrm{In}$ & 110.280 & 111.219 \\
$\mathrm{Tl}$ & 110.251 & 112.092
\end{tabular}

${ }^{a}$ def2-svp for C, H. def2-tzvp for all other elements.

Table S7. Mayer bond orders for the E-E bond in the dimer complexes $\left[\left({ }^{\mathrm{Dip}} \mathrm{LE}\right)_{2}\right]$ at different levels of theory

\begin{tabular}{lrrrrr}
\hline $\mathrm{E}=$ & pbe0/def2-svp & pbe0/def2-tzvp $^{\mathrm{a}}$ & $\begin{array}{c}\text { Level of theory } \\
\text { pbe0/def2-tzvp }^{\mathrm{b}}\end{array}$ & pbe0/cc-pvdz $^{\mathrm{b}}$ & pbe0/cc-pvtz $^{\mathrm{b}}$ \\
\hline $\mathrm{Al}$ & 0.860733 & 1.030952 & 1.132335 & 0.941592 & 0.778666 \\
$\mathrm{Ga}$ & 0.661094 & 0.781819 & 0.841087 & & \\
$\mathrm{In}$ & 0.362607 & 0.412415 & 0.537896 & & \\
$\mathrm{Tl}$ & 0.104623 & 0.105849 & & & \\
\hline
\end{tabular}

a def2-svp for C, H. def2-tzvp for all other elements. ${ }^{b}$ Optimised at the pbe0/def2-tzvpa level.

Table S8. Calculated energies for the dimers $\left[\left({ }^{\mathrm{Dip}} \mathrm{LE}\right)_{2}\right] . \Delta \varepsilon, \Delta \mathrm{H}$, and $\Delta \mathrm{G}$ are given relative to the monomer.

\begin{tabular}{|c|c|c|c|c|c|c|c|c|c|c|}
\hline $\mathrm{E}=$ & Theory level & $\varepsilon$ (a.u.) & $\mathrm{CP}^{\mathrm{b}}$ (a.u.) & $\mathrm{ZPE}_{\text {corr }}$ (a.u.) & $\mathrm{H}_{\text {corr }}$ (a.u.) & $\mathrm{S}(\mathrm{J} / \mathrm{mol} \mathrm{K})$ & $\Delta \varepsilon(\mathrm{kJ} / \mathrm{mol})$ & $\Delta \mathrm{ZPE}(\mathrm{kJ} / \mathrm{mol})$ & $\Delta \mathrm{H}(\mathrm{kJ} / \mathrm{mol})$ & $\Delta \mathrm{G}(\mathrm{kJ} / \mathrm{mol})$ \\
\hline \multirow[t]{4}{*}{$\mathrm{Al}$} & pbe $0 /$ def2-svp & -4176.81892889 & (not applied) & 1.437160 & 1.521376 & 1825.8 & -73.757 & -67.902 & -63.900 & -4.6879 \\
\hline & pbe0/def2-tzvp ${ }^{\mathrm{a}}$ & -4177.48935842 & 0.005260970086 & 1.436404 & 1.520957 & 1845.0 & -56.336 & -52.382 & -46.740 & 1.2025 \\
\hline & pbe0/def2-tzvp ${ }^{a}$ & -4177.48935842 & (not applied) & 1.436404 & 1.520957 & 1845.0 & -70.149 & -66.195 & -60.553 & -12.610 \\
\hline & $\begin{array}{l}\text { pbe0-GD3- } \\
\text { BJ/def2-tzvp }\end{array}$ & -4177.79398548 & 0.006925108380 & 1.439028 & 1.522800 & 1798.6 & -142.90 & -136.88 & -127.37 & -87.713 \\
\hline \multirow[t]{4}{*}{$\mathrm{Ga}^{69,69}$} & pbe $0 /$ def $2-s v p$ & -7541.04586818 & (not applied) & 1.434625 & 1.520463 & 1876.4 & -44.818 & -41.124 & -29.850 & 0.0899 \\
\hline & pbe0/def2-tzvp ${ }^{\mathrm{a}}$ & -7542.04159681 & 0.005125342021 & 1.434401 & 1.520290 & 1883.7 & -30.110 & -26.563 & -15.282 & 13.192 \\
\hline & pbe0/def2-tzvp ${ }^{a}$ & -7542.04159681 & (not applied) & 1.434401 & 1.520290 & 1883.7 & -43.567 & -40.020 & -28.739 & -0.2653 \\
\hline & $\begin{array}{l}\text { pbe0-GD3- } \\
\text { BJ/def2-tzvp }\end{array}$ & -7542.34600342 & 0.007155634257 & 1.437282 & 1.522153 & 1827.5 & -113.21 & -106.41 & -96.835 & -57.390 \\
\hline \multirow[t]{4}{*}{ In } & pbe0/def2-svp & -4072.61024029 & (not applied) & 1.432840 & 1.519581 & 1945.4 & -24.136 & -22.561 & -19.308 & 31.974 \\
\hline & pbe0/def2-tzvp ${ }^{a}$ & -4073.12616128 & 0.003406926824 & 1.432303 & 1.518250 & 1912.6 & -15.582 & -15.044 & -13.603 & 41.317 \\
\hline & pbe0/def2-tzvp ${ }^{a}$ & -4073.12616128 & (not applied) & 1.432303 & 1.518250 & 1912.6 & -24.527 & -23.989 & -22.547 & 32.372 \\
\hline & $\begin{array}{l}\text { pbe0-GD3- } \\
\text { BJ/def2-tzvp }\end{array}$ & -4073.42820235 & 0.005629233950 & 1.434892 & 1.519007 & 1825.0 & -77.477 & -76.002 & -78.194 & -3.6565 \\
\hline \multirow[t]{4}{*}{$\mathrm{Tl}^{205,205}$} & pbe0/def2-svp & -4037.35524546 & (not applied) & 1.431636 & 1.516136 & 1872.0 & -12.127 & -11.838 & -14.925 & 57.704 \\
\hline & pbe0/def2-tzvp ${ }^{a}$ & -4037.86935908 & 0.003259610853 & 1.432442 & 1.519282 & 1931.9 & -5.2928 & -0.8557 & 1.4075 & 62.021 \\
\hline & pbe0/def2-tzvp ${ }^{a}$ & -4037.86935908 & (not applied) & 1.432442 & 1.519282 & 1931.9 & -13.851 & -9.4124 & -7.1492 & 53.445 \\
\hline & $\begin{array}{l}\text { pbe0-GD3- } \\
\text { BJ/def2-tzvp }\end{array}$ & -4038.17109627 & 0.005340943011 & 1.434118 & 1.519525 & 1905.1 & -59.844 & -58.158 & -57.615 & -2.7255 \\
\hline
\end{tabular}

${ }^{\mathrm{a}}$ def2-svp for C, H. def2-tzvp for all other elements. ${ }^{\mathrm{b}}$ Counterpoise correction factor, if applicable. 
3.2 Selected molecular orbitals for $\left[{ }^{\mathrm{Dip}} \mathrm{LE}:\right]$ and $\left[\left({ }^{\mathrm{Dip}} \mathrm{LE}\right)_{2}\right](\mathrm{E}=\mathrm{Al}$, Ga, In, Tl $)$ at pbe0/def2$\operatorname{tzvp}(\mathrm{E}, \mathrm{N}, \mathrm{P}), \operatorname{def} 2-\operatorname{svp}(\mathrm{C}, \mathrm{H})$ level of theory (isovalue $=\mathbf{0 . 0 4})$

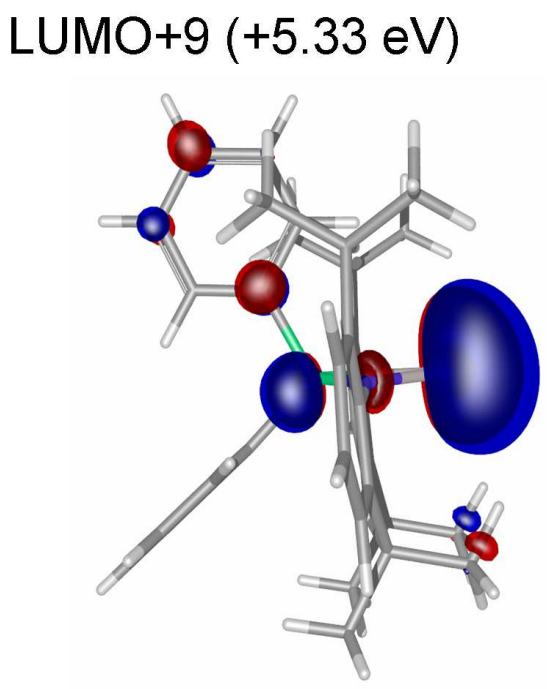

$\mathrm{HOMO}+4(+4.47 \mathrm{eV})$

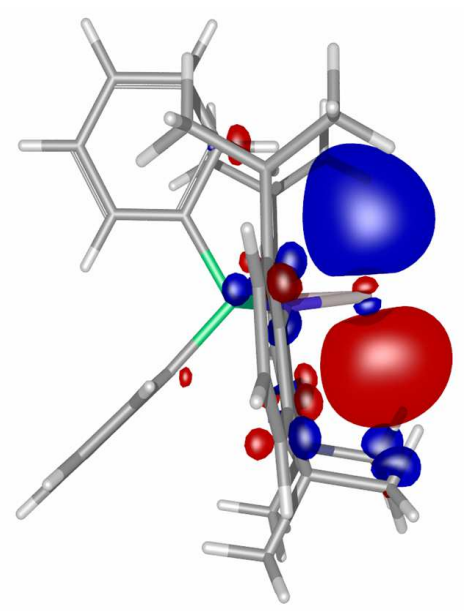

HOMO

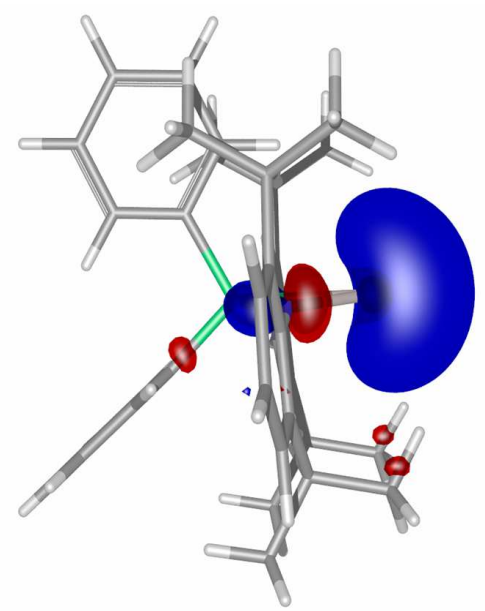

Figure S5. [ $\left.{ }^{\mathrm{Dip}} \mathrm{LAl}:\right]$ 


\section{LUMO+9 (+3.57 eV)}

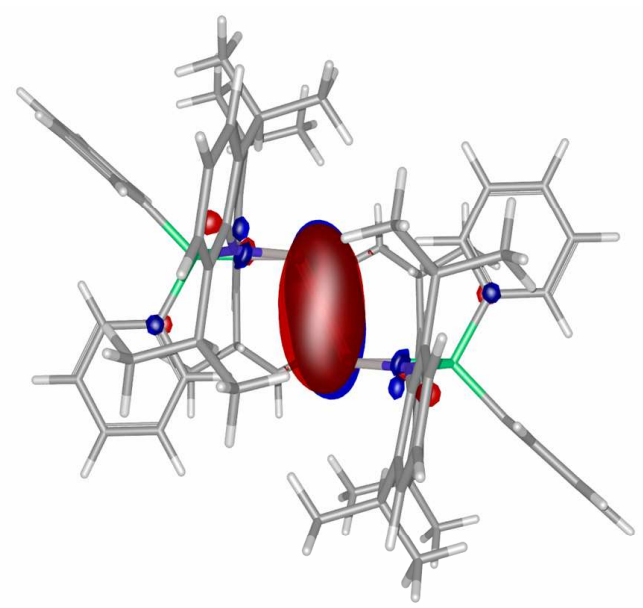

HOMO

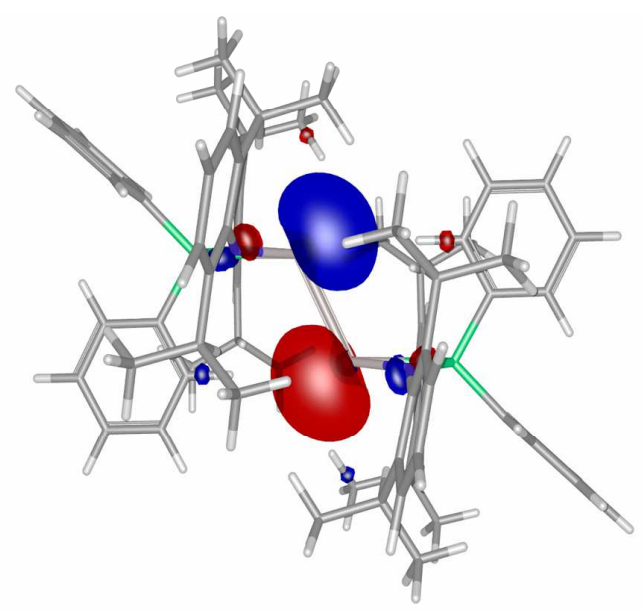

HOMO-11 (-3.47 eV)

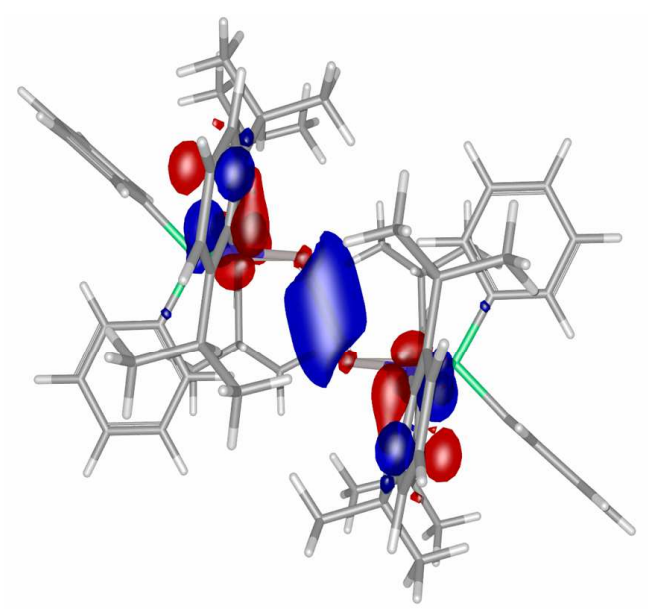

LUMO+6 (+3.12 eV)

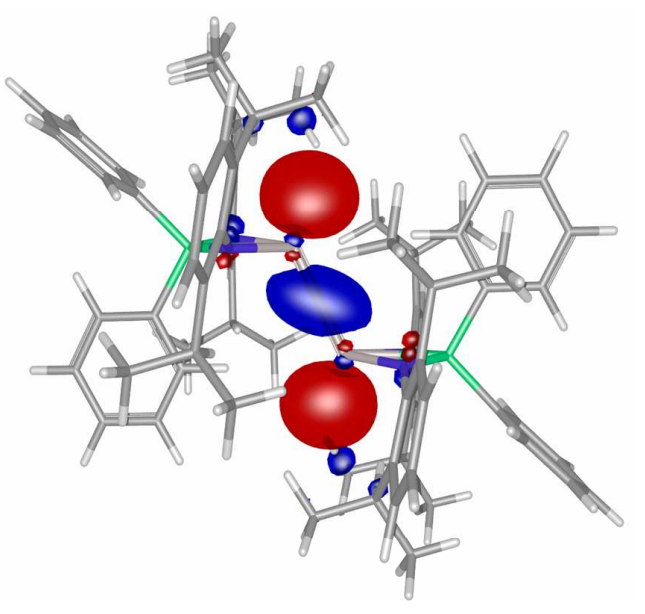

HOMO-1 (-2.22 eV)

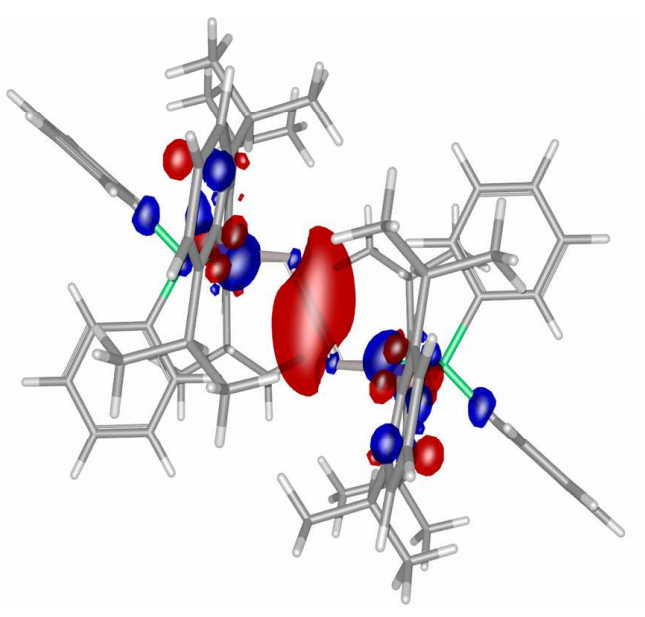

HOMO-15 (-3.96 eV)

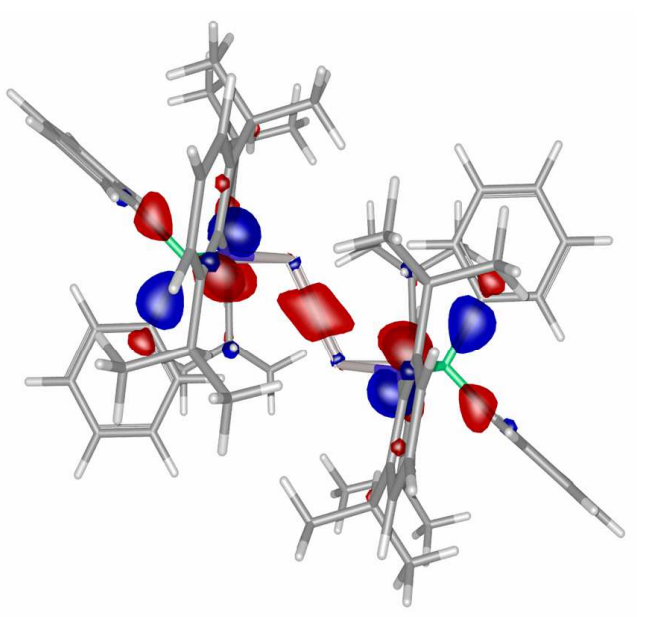

Figure S6. $\left[\left({ }^{\mathrm{Dip}} \mathrm{LAl}\right)_{2}\right]$. The LUMO+9 represents a $\pi$-bonding interaction. 


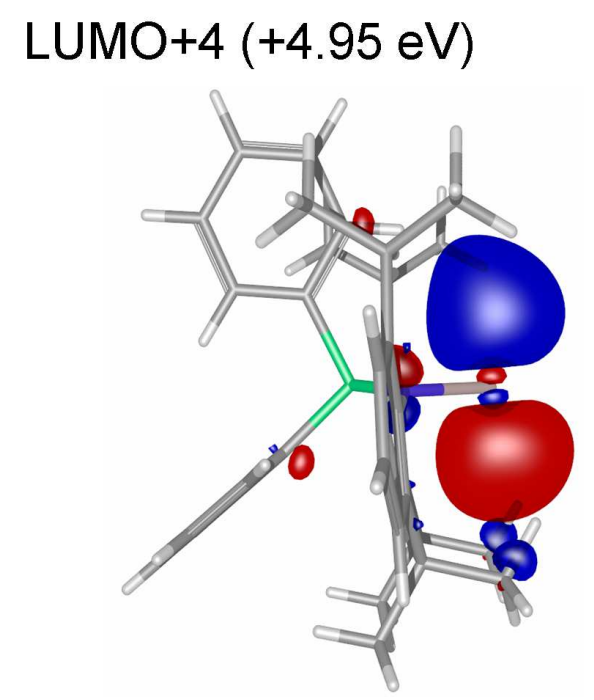

HOMO

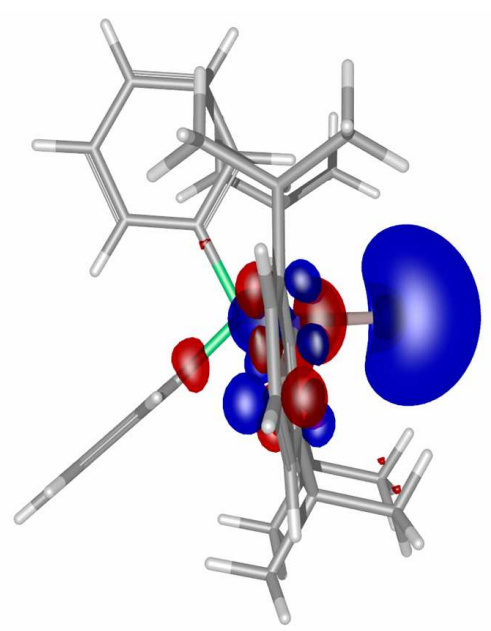

HOMO-5 (-1.59 eV)

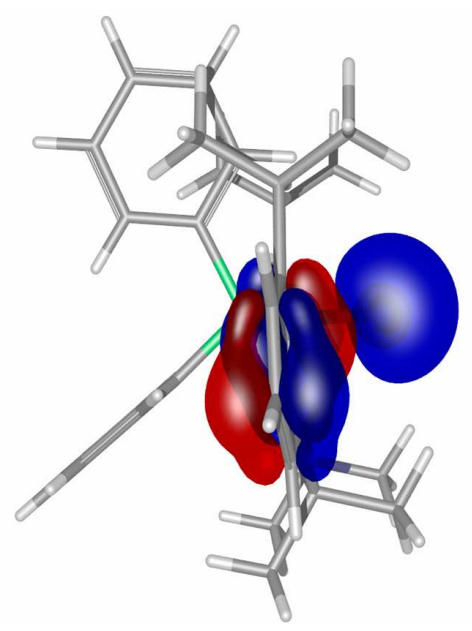

Figure S7. [ ${ }^{\text {Dip }}$ Ga:] (1, c.f. Figure 5) 
LUMO+9 (+4.47 eV)

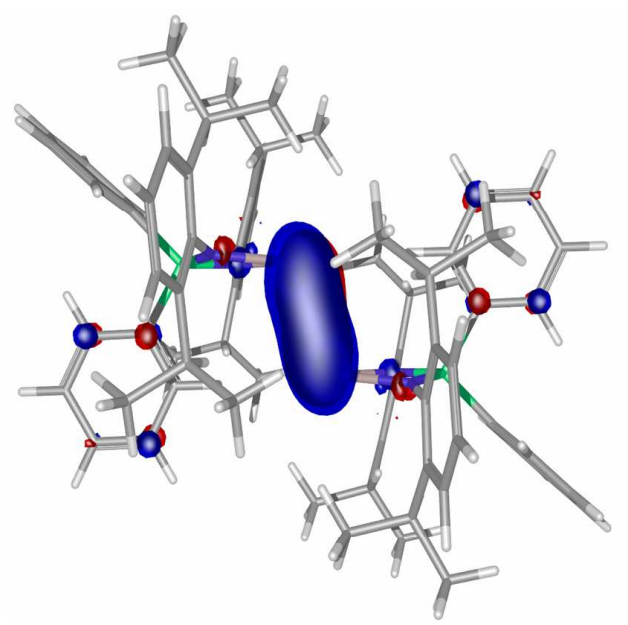

LUMO+2 (+3.58 eV)

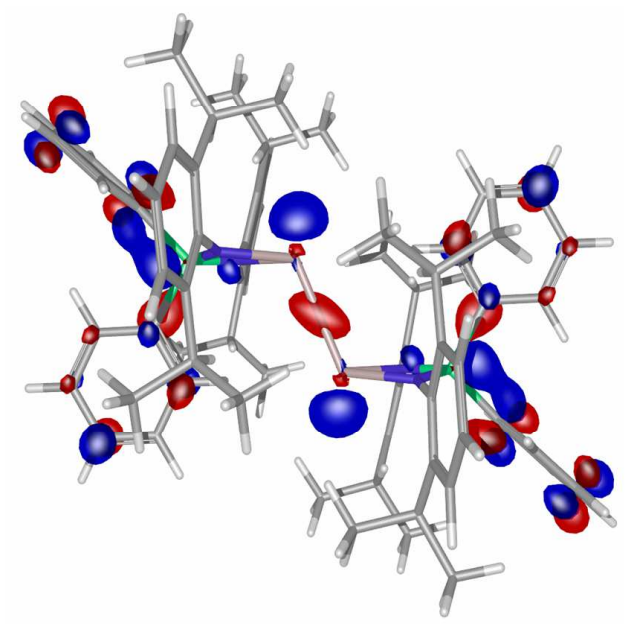

HOMO-1 (-1.30 eV)

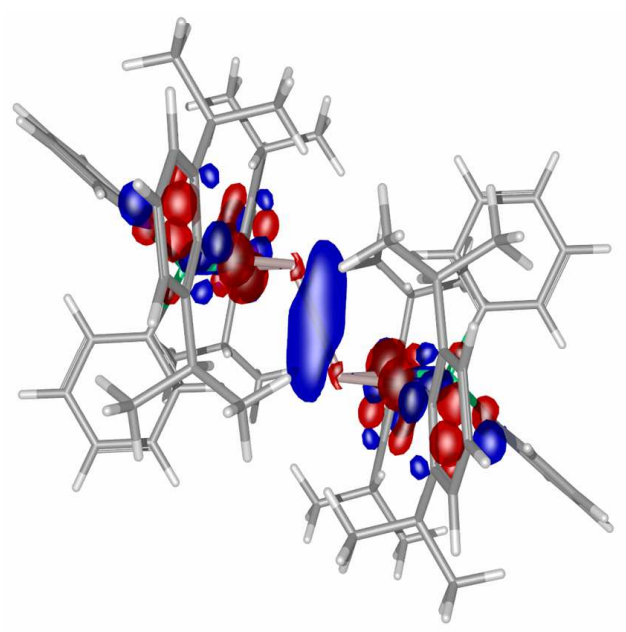

LUMO+4 (+3.79 eV)

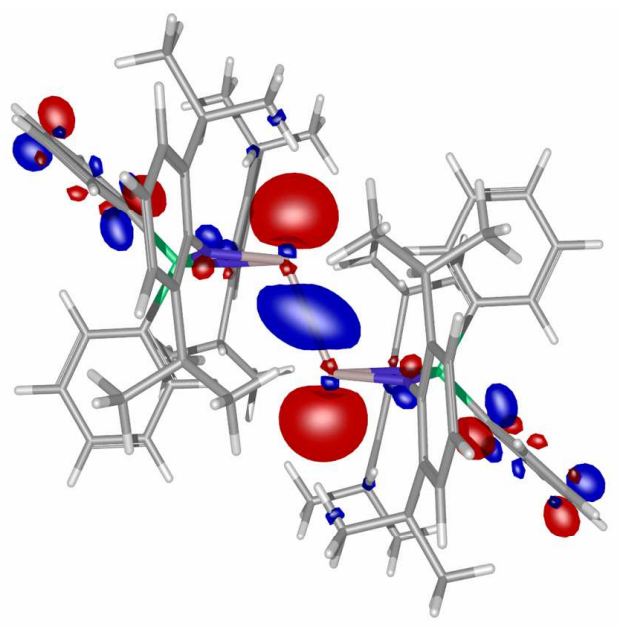

HOMO

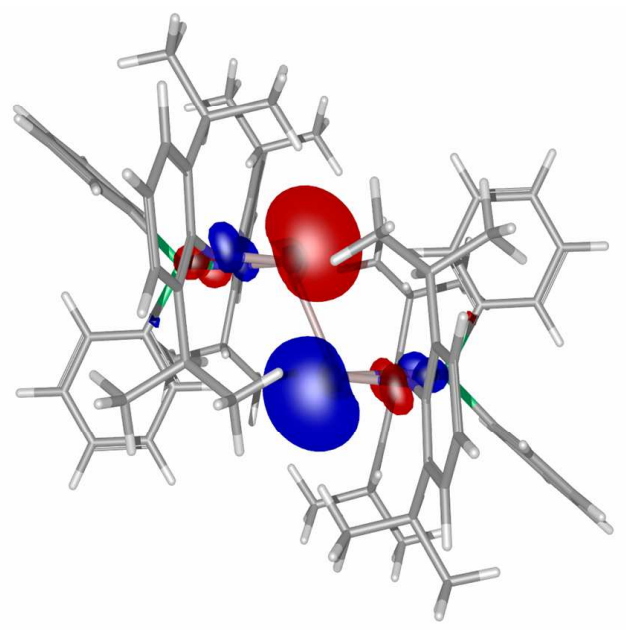

HOMO-17 (-3.03 eV)

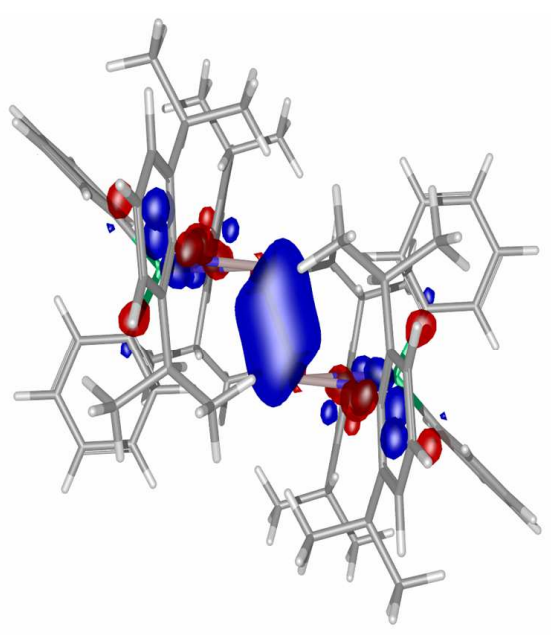

Figure S8. $\left[\left({ }^{\mathrm{Dip}} \mathrm{LGa}\right)_{2}\right]\left(\mathbf{1}_{2}\right.$, c.f. Figure 5). 
LUMO+10 (+6.60 eV)

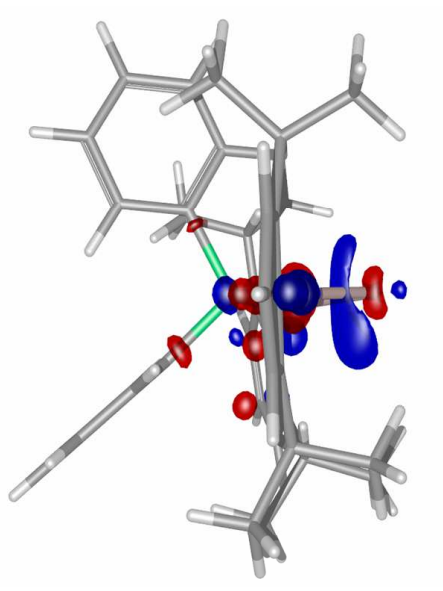

LUMO+2 (+4.80 eV)

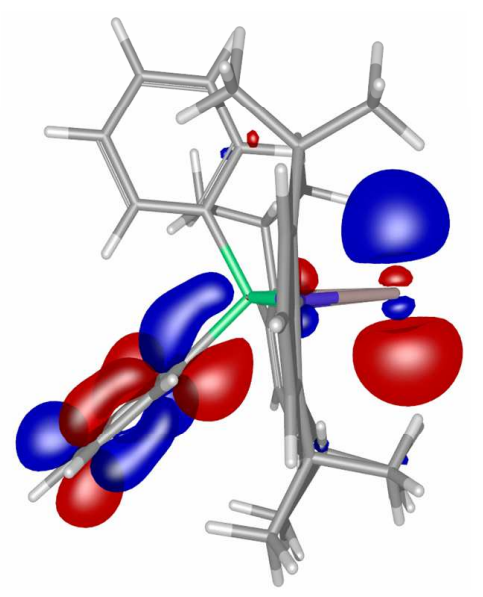

HOMO

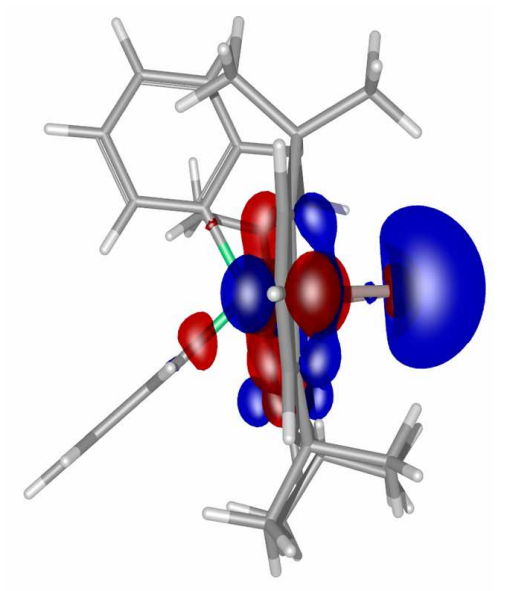

LUMO+5 (+5.40 eV)

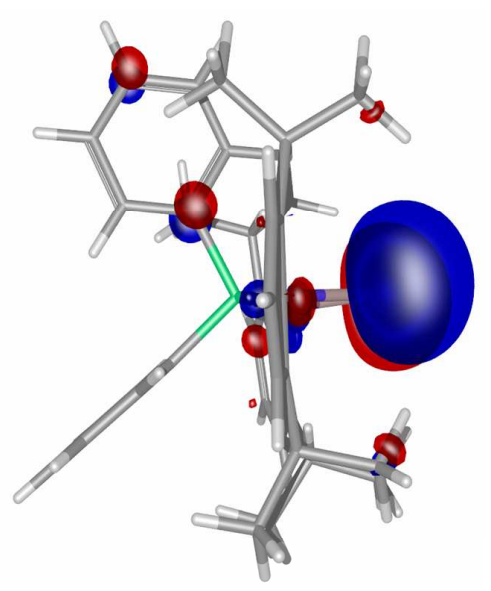

LUMO+1 (+4.61 eV)

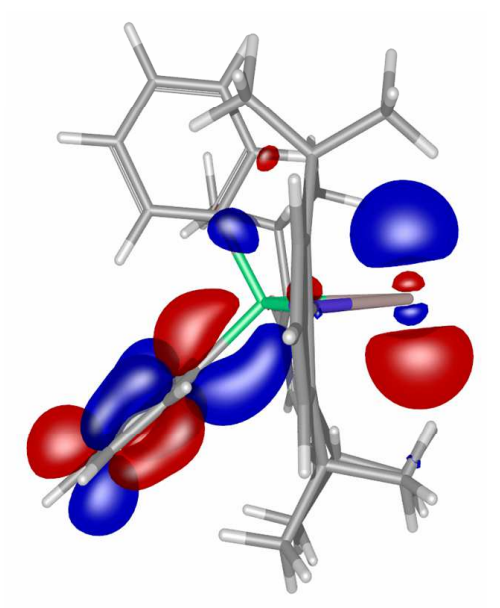

HOMO-6 (-1.52 eV)

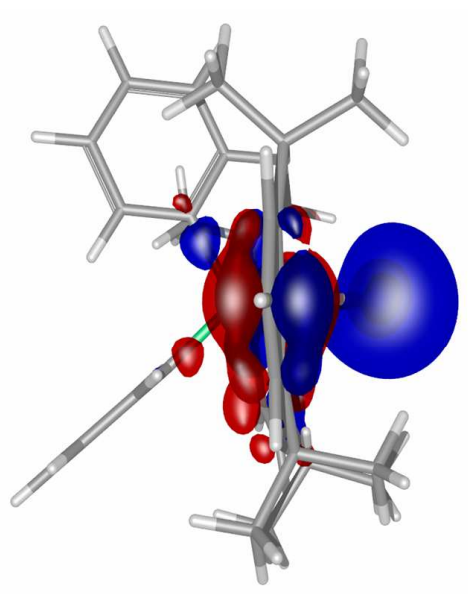

Figure S9. $\left[{ }^{\mathrm{Dip}}\right.$ LIn:] (2) 
LUMO+18 (+5.81 eV)

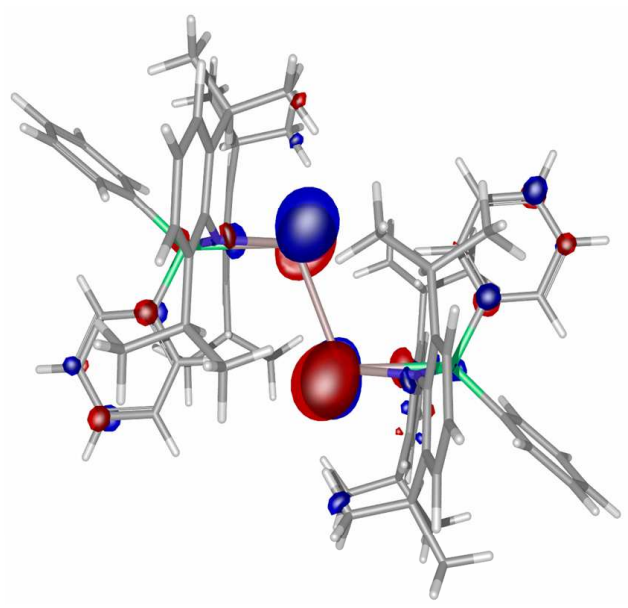

LUMO (+3.73 eV)

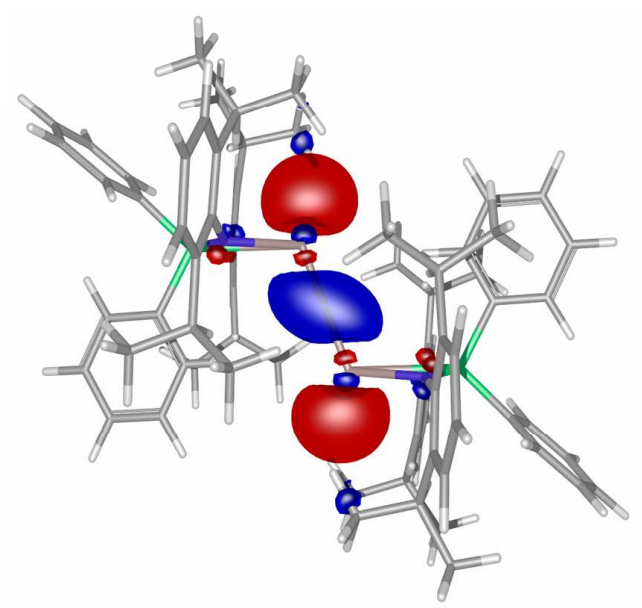

HOMO-1 (-0.76 eV)

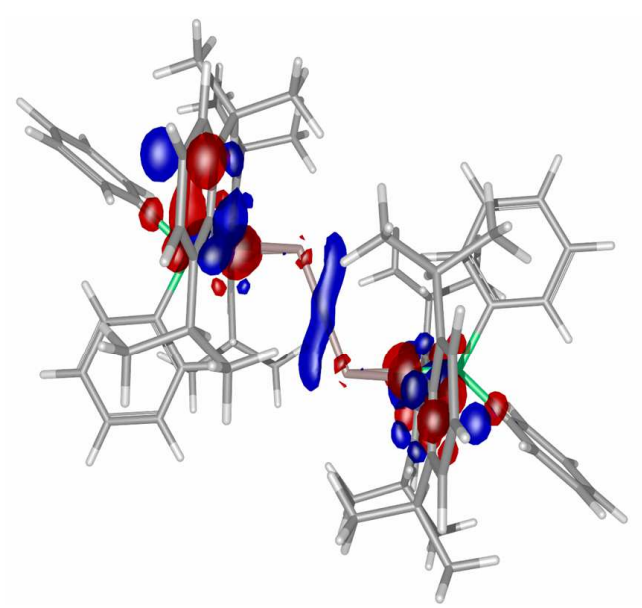

LUMO+5 (+4.45 eV)

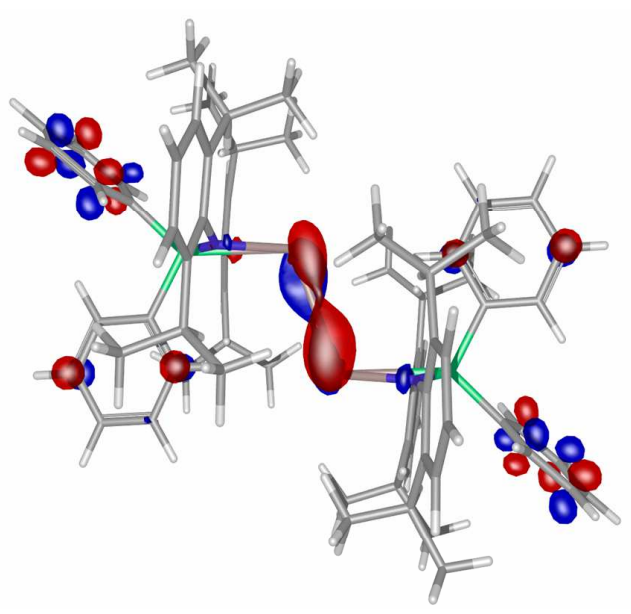

HOMO

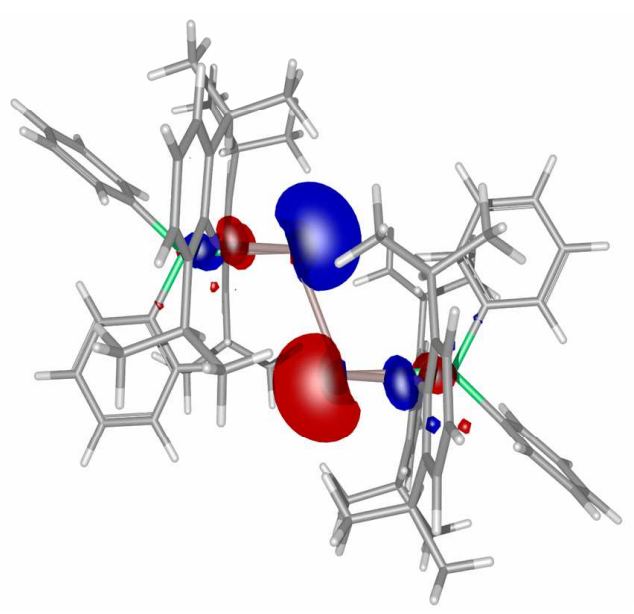

HOMO-17 (-2.47 eV)

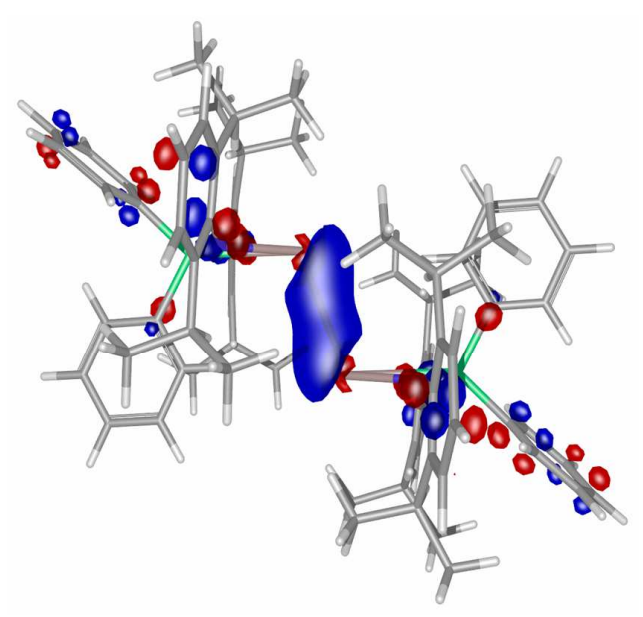

Figure S10. $\left[\left({ }^{\mathrm{Dip}} \mathrm{LIn}\right)_{2}\right]\left(\mathbf{2}_{\mathbf{2}}\right)$ 
LUMO+6 (+5.69 eV)

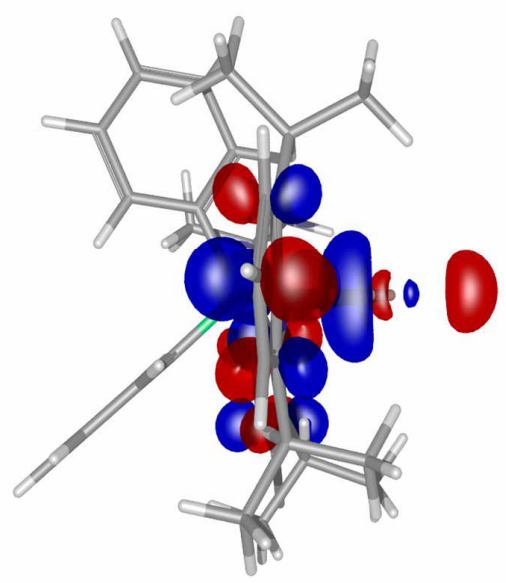

LUMO+2 (+4.99 eV)

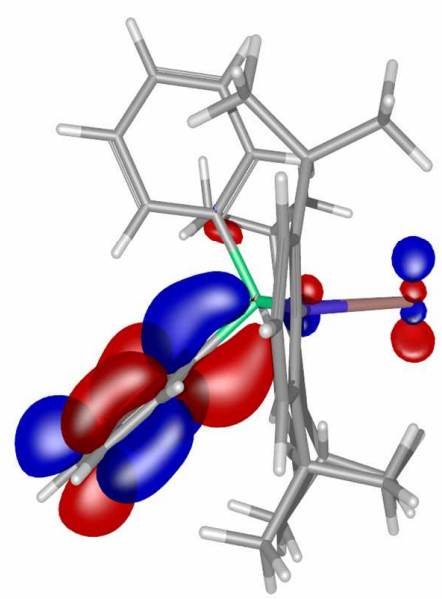

HOMO

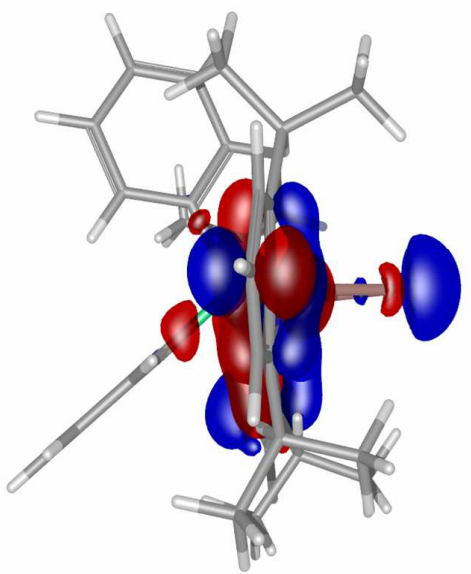

LUMO+4 (+5.40 eV)

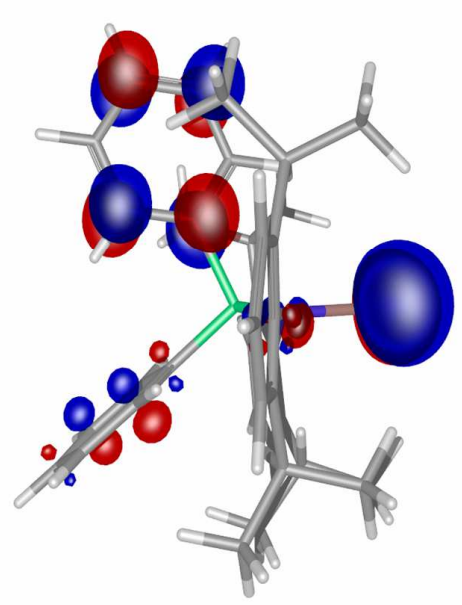

LUMO (+4.68 eV)

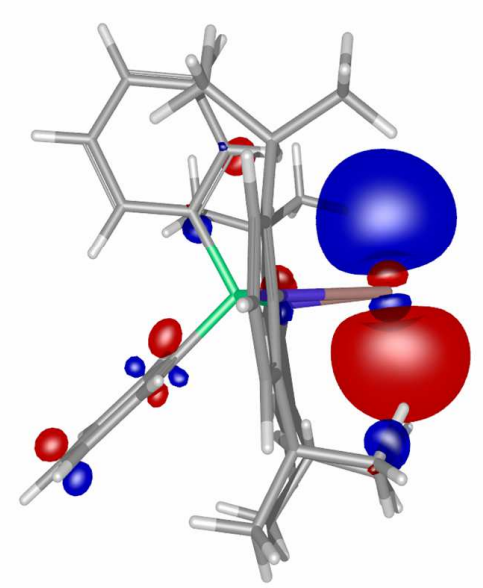

HOMO-10 (-2.12 eV)

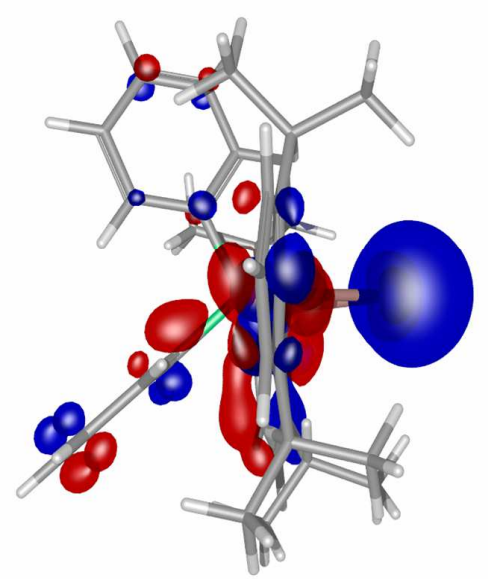

Figure S11. $\left[{ }^{\mathrm{Dip}} \mathrm{LTl}\right.$ :] (3). N.B. The LUMO+1 and LUMO+3 are similar to the LUMO+2. 
LUMO+21 (+6.80 eV)

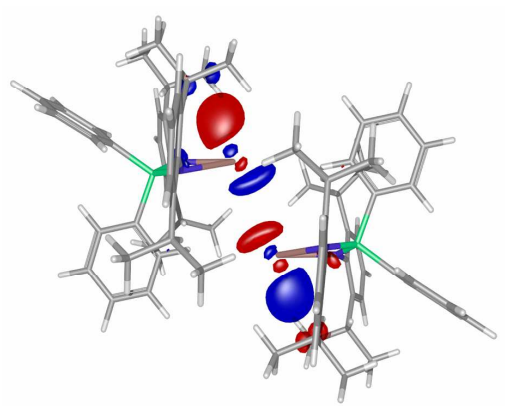

LUMO+6 (+5.15 eV)

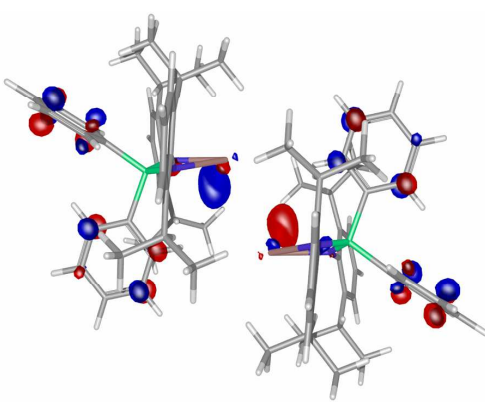

HOMO

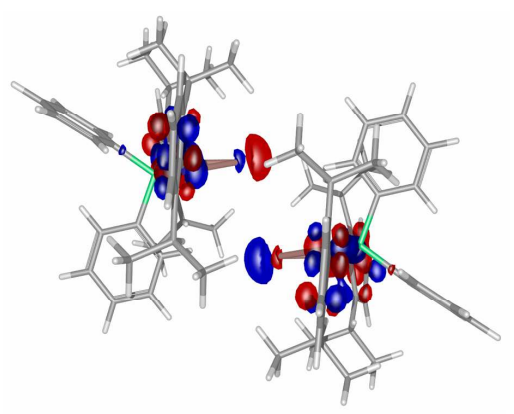

LUMO+12 (+5.55 eV)

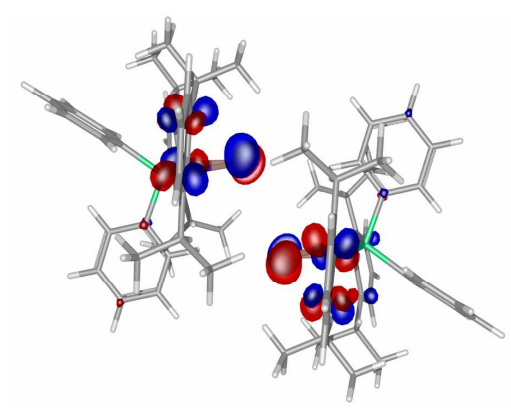

LUMO+1 (+4.68 eV)

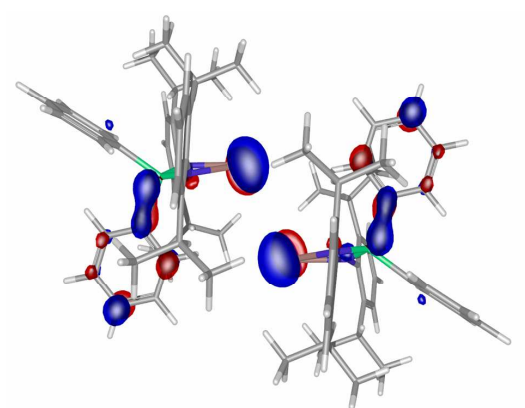

HOMO-18 (-1.91 eV)

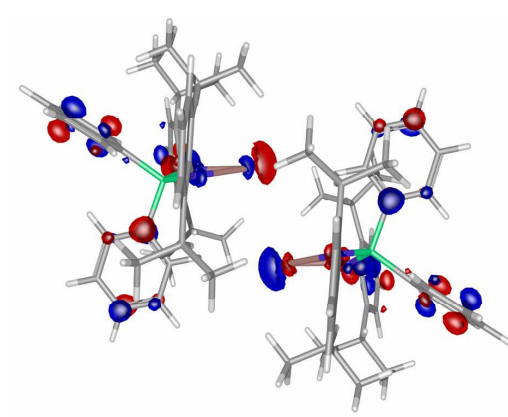

LUMO+7 (+5.18 eV)

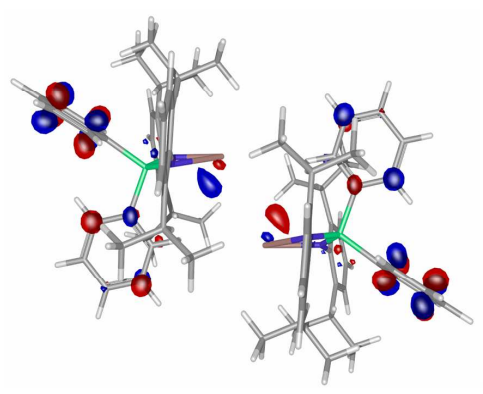

LUMO (+4.03 eV)

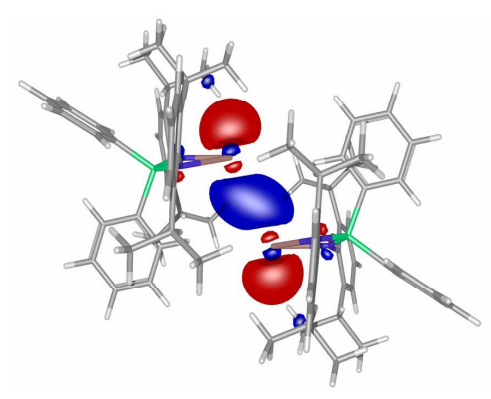

HOMO-21 (-2.41 eV)

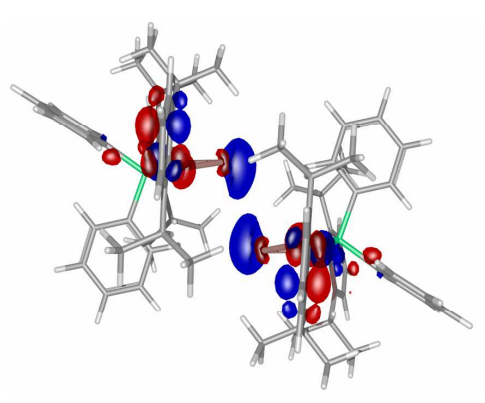

Figure S12. $\left[\left({ }^{\mathrm{Dip}} \mathrm{LTl}\right)_{2}\right]\left(\mathbf{3}_{\mathbf{2}}\right)$ 


\subsection{Atomic coordinates for optimized geometries at various levels of theory}

[ ${ }^{\text {Dip }}$ LAl:], singlet, pbe0/def2-tzvp 84

\begin{tabular}{|c|c|c|c|}
\hline $\mathrm{Al}$ & 0.00000 & -0.19752 & -2.44872 \\
\hline $\mathrm{P}$ & 0.00000 & 0.26882 & 0.18140 \\
\hline $\mathrm{N}$ & 1.20438 & -0.01590 & -0.85821 \\
\hline C & 0.00000 & 1.97927 & 0.79936 \\
\hline $\mathrm{N}$ & -1.20438 & -0.01590 & -0.85821 \\
\hline $\mathrm{C}$ & 0.00000 & 3.02269 & -0.13892 \\
\hline $\mathrm{H}$ & 0.00000 & 2.79195 & -1.20851 \\
\hline $\mathrm{C}$ & 0.00000 & 4.34980 & 0.27426 \\
\hline $\mathrm{H}$ & 0.00000 & 5.15041 & -0.46926 \\
\hline $\mathrm{C}$ & 0.00000 & 4.65660 & 1.63686 \\
\hline $\mathrm{H}$ & 0.00000 & 5.69959 & 1.96285 \\
\hline C & 0.00000 & 3.63062 & 2.57765 \\
\hline $\mathrm{H}$ & 0.00000 & 3.86416 & 3.64497 \\
\hline C & 0.00000 & 2.29774 & 2.16284 \\
\hline $\mathrm{H}$ & 0.00000 & 1.50380 & 2.91138 \\
\hline C & 0.00000 & -0.70072 & 1.70767 \\
\hline $\mathrm{C}$ & 1.21144 & -1.02827 & 2.33310 \\
\hline $\mathrm{H}$ & 2.16181 & -0.76243 & 1.86536 \\
\hline $\mathrm{C}$ & 1.20751 & -1.69440 & 3.55748 \\
\hline $\mathrm{H}$ & 2.15577 & -1.94934 & 4.03670 \\
\hline $\mathrm{C}$ & 0.00000 & -2.03035 & 4.16890 \\
\hline $\mathrm{H}$ & 0.00000 & -2.55187 & 5.12927 \\
\hline $\mathrm{C}$ & -1.20751 & -1.69440 & 3.55748 \\
\hline $\mathrm{H}$ & -2.15577 & -1.94934 & 4.03670 \\
\hline C & -1.21144 & -1.02827 & 2.33310 \\
\hline $\mathrm{H}$ & -2.16181 & -0.76243 & 1.86536 \\
\hline $\mathrm{C}$ & 2.58296 & -0.28763 & -0.67543 \\
\hline $\mathrm{C}$ & 3.54857 & 0.74746 & -0.70400 \\
\hline $\mathrm{C}$ & 4.89794 & 0.42598 & -0.51860 \\
\hline $\mathrm{H}$ & 5.63959 & 1.22936 & -0.52989 \\
\hline $\mathrm{C}$ & 5.31498 & -0.88454 & -0.33550 \\
\hline $\mathrm{H}$ & 6.37329 & -1.11461 & -0.19017 \\
\hline $\mathrm{C}$ & 4.37077 & -1.90461 & -0.36417 \\
\hline $\mathrm{H}$ & 4.70001 & -2.94043 & -0.25459 \\
\hline C & 3.01066 & -1.63803 & -0.54059 \\
\hline $\mathrm{C}$ & 3.18480 & 2.19292 & -0.97654 \\
\hline $\mathrm{H}$ & 2.09744 & 2.22499 & -1.11011 \\
\hline C & 3.81096 & 2.68567 & -2.28303 \\
\hline $\mathrm{H}$ & 4.91157 & 2.69693 & -2.22974 \\
\hline $\mathrm{H}$ & 3.48042 & 3.71267 & -2.50756 \\
\hline $\mathrm{H}$ & 3.52391 & 2.04308 & -3.12918 \\
\hline C & 3.53601 & 3.11106 & 0.19378 \\
\hline $\mathrm{H}$ & 3.03840 & 2.78908 & 1.12132 \\
\hline $\mathrm{H}$ & 3.21920 & 4.14523 & -0.01427 \\
\hline $\mathrm{H}$ & 4.62160 & 3.12849 & 0.38317 \\
\hline $\mathrm{C}$ & 2.04453 & -2.80362 & -0.65946 \\
\hline $\mathrm{H}$ & 1.03575 & -2.41508 & -0.45438 \\
\hline $\mathrm{C}$ & 2.04630 & -3.34240 & -2.09359 \\
\hline $\mathrm{H}$ & 1.77573 & -2.56044 & -2.82138 \\
\hline $\mathrm{H}$ & 1.32677 & -4.16958 & -2.20449 \\
\hline $\mathrm{H}$ & 3.04489 & -3.71982 & -2.36705 \\
\hline C & 2.30439 & -3.93060 & 0.33885 \\
\hline $\mathrm{H}$ & 3.25130 & -4.45520 & 0.13509 \\
\hline $\mathrm{H}$ & 1.50256 & -4.68291 & 0.27535 \\
\hline $\mathrm{H}$ & 2.33704 & -3.56082 & 1.37438 \\
\hline C & -2.58296 & -0.28763 & -0.67543 \\
\hline $\mathrm{C}$ & -3.54857 & 0.74746 & -0.70400 \\
\hline C & -4.89794 & 0.42598 & -0.51860 \\
\hline $\mathrm{H}$ & -5.63959 & 1.22936 & -0.52989 \\
\hline C & -5.31498 & -0.88454 & -0.33550 \\
\hline $\mathrm{H}$ & -6.37329 & -1.11460 & -0.19017 \\
\hline C & -4.37077 & -1.90461 & -0.36418 \\
\hline $\mathrm{H}$ & -4.70001 & -2.94043 & -0.25459 \\
\hline C & -3.01066 & -1.63803 & -0.54059 \\
\hline $\mathrm{C}$ & -3.18480 & 2.19292 & -0.97654 \\
\hline $\mathrm{H}$ & -2.09744 & 2.22499 & -1.11011 \\
\hline C & -3.81096 & 2.68567 & -2.28303 \\
\hline $\mathrm{H}$ & -3.52391 & 2.04308 & -3.12918 \\
\hline $\mathrm{H}$ & -3.48042 & 3.71267 & -2.50756 \\
\hline $\mathrm{H}$ & -4.91157 & 2.69694 & -2.22973 \\
\hline $\mathrm{C}$ & -3.53601 & 3.11106 & 0.19378 \\
\hline $\mathrm{H}$ & -4.62160 & 3.12849 & 0.38317 \\
\hline $\mathrm{H}$ & -3.21920 & 4.14523 & -0.01427 \\
\hline $\mathrm{H}$ & -3.03840 & 2.78907 & 1.12132 \\
\hline $\mathrm{C}$ & -2.04453 & -2.80362 & -0.65946 \\
\hline $\mathrm{H}$ & -1.03575 & -2.41508 & -0.45438 \\
\hline C & -2.04630 & -3.34240 & -2.09359 \\
\hline $\mathrm{H}$ & -3.04489 & -3.71982 & -2.36705 \\
\hline $\mathrm{H}$ & -1.32677 & -4.16958 & -2.20449 \\
\hline $\mathrm{H}$ & -1.77573 & -2.56044 & -2.82138 \\
\hline C & -2.30439 & -3.93060 & 0.33885 \\
\hline $\mathrm{H}$ & -2.33704 & -3.56082 & 1.37438 \\
\hline $\mathrm{H}$ & -1.50256 & -4.68291 & 0.27535 \\
\hline $\mathrm{H}$ & -3.25130 & -4.45520 & 0.13509 \\
\hline
\end{tabular}

[ ${ }^{\text {Dip }}$ LAl:], singlet, pbe0-gd3-bj/def2-tzvp 84$$
\text { (1) }
$$

(1)


[ ${ }^{\text {Dip }}$ LAl:], triplet, pbe0/def2-tzvp 84

\begin{tabular}{|c|c|c|c|}
\hline $\mathrm{Al}$ & -0.16255 & -0.67066 & -2.15064 \\
\hline$P$ & 0.03560 & 0.45103 & 0.17744 \\
\hline $\mathrm{N}$ & -1.25037 & 0.08831 & -0.88407 \\
\hline $\mathrm{C}$ & 0.41576 & 2.12484 & 0.45249 \\
\hline $\mathrm{N}$ & 1.12850 & -0.28098 & -0.95698 \\
\hline $\mathrm{C}$ & 0.43869 & 3.00569 & -0.67544 \\
\hline $\mathrm{H}$ & 0.28098 & 2.60602 & -1.68110 \\
\hline $\mathrm{C}$ & 0.63723 & 4.36910 & -0.51530 \\
\hline $\mathrm{H}$ & 0.64531 & 5.01246 & -1.39986 \\
\hline $\mathrm{C}$ & 0.83409 & 4.92558 & 0.75297 \\
\hline $\mathrm{H}$ & 1.00140 & 5.99775 & 0.87187 \\
\hline $\mathrm{C}$ & 0.80446 & 4.07389 & 1.87451 \\
\hline $\mathrm{H}$ & 0.94141 & 4.49003 & 2.87628 \\
\hline $\mathrm{C}$ & 0.59020 & 2.71584 & 1.73930 \\
\hline $\mathrm{H}$ & 0.54035 & 2.09300 & 2.63340 \\
\hline $\mathrm{C}$ & 0.00137 & -0.43544 & 1.71820 \\
\hline $\mathrm{C}$ & -1.10729 & -1.21333 & 2.10872 \\
\hline $\mathrm{H}$ & -1.99184 & -1.25993 & 1.47240 \\
\hline $\mathrm{C}$ & -1.09963 & -1.89384 & 3.32114 \\
\hline $\mathrm{H}$ & -1.97462 & -2.48086 & 3.61185 \\
\hline $\mathrm{C}$ & 0.00523 & -1.81449 & 4.17398 \\
\hline $\mathrm{H}$ & 0.00340 & -2.34536 & 5.12877 \\
\hline $\mathrm{C}$ & 1.11331 & -1.05542 & 3.79251 \\
\hline $\mathrm{H}$ & 1.98945 & -0.99884 & 4.44350 \\
\hline $\mathrm{C}$ & 1.12458 & -0.38508 & 2.57317 \\
\hline $\mathrm{H}$ & 2.01480 & 0.17066 & 2.27115 \\
\hline $\mathrm{C}$ & -2.64082 & -0.03372 & -0.64055 \\
\hline $\mathrm{C}$ & -3.44967 & 1.07488 & -0.29232 \\
\hline $\mathrm{C}$ & -4.81416 & 0.87227 & -0.05603 \\
\hline $\mathrm{H}$ & -5.43061 & 1.73036 & 0.22431 \\
\hline $\mathrm{C}$ & -5.40418 & -0.37622 & -0.18527 \\
\hline $\mathrm{H}$ & -6.47304 & -0.50810 & -0.00146 \\
\hline $\mathrm{C}$ & -4.61689 & -1.45609 & -0.56605 \\
\hline $\mathrm{H}$ & -5.08064 & -2.43804 & -0.68859 \\
\hline $\mathrm{C}$ & -3.24532 & -1.31584 & -0.79100 \\
\hline $\mathrm{C}$ & -2.91152 & 2.48678 & -0.19708 \\
\hline $\mathrm{H}$ & -1.85242 & 2.45664 & -0.47272 \\
\hline $\mathrm{C}$ & -3.60048 & 3.42215 & -1.19295 \\
\hline $\mathrm{H}$ & -4.66926 & 3.55964 & -0.96144 \\
\hline $\mathrm{H}$ & -3.12608 & 4.41594 & -1.16918 \\
\hline $\mathrm{H}$ & -3.52983 & 3.03662 & -2.22185 \\
\hline C & -2.99070 & 3.03414 & 1.22809 \\
\hline $\mathrm{H}$ & -2.47003 & 2.37325 & 1.93697 \\
\hline $\mathrm{H}$ & -2.51498 & 4.02526 & 1.28611 \\
\hline $\mathrm{H}$ & -4.03548 & 3.13744 & 1.56531 \\
\hline $\mathrm{C}$ & -2.47529 & -2.54871 & -1.23702 \\
\hline $\mathrm{H}$ & -1.39269 & -2.33388 & -1.11550 \\
\hline $\mathrm{C}$ & -2.73950 & -2.85659 & -2.71343 \\
\hline $\mathrm{H}$ & -2.51928 & -1.99018 & -3.35928 \\
\hline $\mathrm{H}$ & -2.12850 & -3.70505 & -3.06103 \\
\hline $\mathrm{H}$ & -3.79929 & -3.11176 & -2.86932 \\
\hline $\mathrm{C}$ & -2.72984 & -3.78098 & -0.36828 \\
\hline $\mathrm{H}$ & -3.77071 & -4.12935 & -0.45255 \\
\hline $\mathrm{H}$ & -2.08267 & -4.61366 & -0.68541 \\
\hline $\mathrm{H}$ & -2.52569 & -3.57657 & 0.69288 \\
\hline $\mathrm{C}$ & 2.44858 & -0.68281 & -0.64383 \\
\hline C & 3.54515 & 0.19344 & -0.81923 \\
\hline $\mathrm{C}$ & 4.82413 & -0.24620 & -0.45576 \\
\hline $\mathrm{H}$ & 5.67301 & 0.43044 & -0.58492 \\
\hline C & 5.03851 & -1.51951 & 0.05115 \\
\hline $\mathrm{H}$ & 6.04474 & -1.83994 & 0.33247 \\
\hline $\mathrm{C}$ & 3.96275 & -2.39289 & 0.18199 \\
\hline $\mathrm{H}$ & 4.13759 & -3.40239 & 0.55960 \\
\hline $\mathrm{C}$ & 2.66737 & -2.00250 & -0.15974 \\
\hline $\mathrm{C}$ & 3.40159 & 1.55932 & -1.45892 \\
\hline $\mathrm{H}$ & 2.32917 & 1.74105 & -1.59766 \\
\hline $\mathrm{C}$ & 4.05261 & 1.57615 & -2.84477 \\
\hline $\mathrm{H}$ & 3.64035 & 0.78734 & -3.49347 \\
\hline $\mathrm{H}$ & 3.88573 & 2.54639 & -3.33980 \\
\hline $\mathrm{H}$ & 5.14154 & 1.41708 & -2.78344 \\
\hline $\mathrm{C}$ & 3.93768 & 2.68467 & -0.57591 \\
\hline $\mathrm{H}$ & 5.02033 & 2.58411 & -0.39501 \\
\hline $\mathrm{H}$ & 3.77065 & 3.66151 & -1.05522 \\
\hline $\mathrm{H}$ & 3.42610 & 2.70476 & 0.39783 \\
\hline $\mathrm{C}$ & 1.53447 & -3.01160 & -0.09204 \\
\hline $\mathrm{H}$ & 0.60841 & -2.44757 & 0.09485 \\
\hline C & 1.38847 & -3.72417 & -1.44007 \\
\hline $\mathrm{H}$ & 2.29642 & -4.30025 & -1.67927 \\
\hline $\mathrm{H}$ & 0.53327 & -4.41942 & -1.43787 \\
\hline $\mathrm{H}$ & 1.24445 & -3.01100 & -2.27329 \\
\hline $\mathrm{C}$ & 1.65805 & -4.02247 & 1.04424 \\
\hline $\mathrm{H}$ & 1.78887 & -3.51792 & 2.01284 \\
\hline $\mathrm{H}$ & 0.74580 & -4.63614 & 1.10438 \\
\hline $\mathrm{H}$ & 2.50242 & -4.71473 & 0.89772 \\
\hline
\end{tabular}


[( ${ }^{\text {Dip }}$ LAl $_{2}$ ], singlet, pbe0/def2-tzvp 168

\begin{tabular}{|c|c|c|c|}
\hline $\mathrm{Al}$ & -1.16463 & 0.16798 & 0.74282 \\
\hline $\mathrm{P}$ & -3.45693 & -0.02764 & -0.57281 \\
\hline $\mathrm{N}$ & -2.66843 & 1.25048 & 0.03533 \\
\hline C & -3.50024 & -0.04223 & -2.38660 \\
\hline $\mathrm{N}$ & -2.47593 & -1.15389 & 0.04803 \\
\hline $\mathrm{C}$ & -2.27650 & -0.08703 & -3.07461 \\
\hline $\mathrm{H}$ & -1.33191 & -0.10197 & -2.51778 \\
\hline $\mathrm{C}$ & -2.25199 & -0.10594 & -4.46500 \\
\hline $\mathrm{H}$ & -1.29145 & -0.14196 & -4.98502 \\
\hline C & -3.44534 & -0.07814 & -5.18945 \\
\hline $\mathrm{H}$ & -3.42391 & -0.09432 & -6.28209 \\
\hline $\mathrm{C}$ & -4.66336 & -0.02877 & -4.51610 \\
\hline $\mathrm{H}$ & -5.60093 & -0.00606 & -5.07672 \\
\hline $\mathrm{C}$ & -4.69289 & -0.01256 & -3.12127 \\
\hline $\mathrm{H}$ & -5.65494 & 0.01919 & -2.60753 \\
\hline $\mathrm{C}$ & -5.20761 & -0.18969 & -0.14536 \\
\hline $\mathrm{C}$ & -6.01487 & 0.95118 & -0.03575 \\
\hline $\mathrm{H}$ & -5.57394 & 1.94642 & -0.12815 \\
\hline C & -7.38452 & 0.82124 & 0.18726 \\
\hline $\mathrm{H}$ & -8.00559 & 1.71611 & 0.27361 \\
\hline C & -7.95980 & -0.44460 & 0.29536 \\
\hline $\mathrm{H}$ & -9.03413 & -0.54401 & 0.46946 \\
\hline C & -7.16398 & -1.58306 & 0.17392 \\
\hline $\mathrm{H}$ & -7.61063 & -2.57710 & 0.25191 \\
\hline $\mathrm{C}$ & -5.79363 & -1.46013 & -0.05078 \\
\hline $\mathrm{H}$ & -5.18179 & -2.35864 & -0.15249 \\
\hline C & -2.99273 & 2.62607 & 0.15118 \\
\hline C & -2.59227 & 3.56191 & -0.83302 \\
\hline $\mathrm{C}$ & -2.91312 & 4.91330 & -0.65648 \\
\hline $\mathrm{H}$ & -2.61225 & 5.63174 & -1.42350 \\
\hline $\mathrm{C}$ & -3.59174 & 5.36081 & 0.46697 \\
\hline $\mathrm{H}$ & -3.83303 & 6.42024 & 0.58396 \\
\hline $\mathrm{C}$ & -3.94658 & 4.44565 & 1.45156 \\
\hline $\mathrm{H}$ & -4.45863 & 4.79955 & 2.34897 \\
\hline $\mathrm{C}$ & -3.65583 & 3.08498 & 1.32481 \\
\hline C & -1.80834 & 3.17057 & -2.07039 \\
\hline $\mathrm{H}$ & -1.56476 & 2.10326 & -1.97849 \\
\hline C & -0.48086 & 3.92515 & -2.16324 \\
\hline $\mathrm{H}$ & -0.63129 & 5.01046 & -2.27996 \\
\hline $\mathrm{H}$ & 0.09496 & 3.57773 & -3.03450 \\
\hline $\mathrm{H}$ & 0.14211 & 3.76855 & -1.26950 \\
\hline C & -2.63441 & 3.36300 & -3.34318 \\
\hline $\mathrm{H}$ & -3.56980 & 2.78441 & -3.31278 \\
\hline $\mathrm{H}$ & -2.06628 & 3.03500 & -4.22763 \\
\hline $\mathrm{H}$ & -2.89888 & 4.42305 & -3.49055 \\
\hline C & -4.00192 & 2.15325 & 2.47468 \\
\hline $\mathrm{H}$ & -4.08529 & 1.13769 & 2.06129 \\
\hline $\mathrm{C}$ & -2.87221 & 2.13322 & 3.50832 \\
\hline $\mathrm{H}$ & -1.92292 & 1.79453 & 3.06174 \\
\hline $\mathrm{H}$ & -3.11471 & 1.45198 & 4.34006 \\
\hline $\mathrm{H}$ & -2.70966 & 3.13919 & 3.92818 \\
\hline C & -5.33295 & 2.47549 & 3.15304 \\
\hline $\mathrm{H}$ & -5.29056 & 3.41812 & 3.72138 \\
\hline $\mathrm{H}$ & -5.59176 & 1.68120 & 3.87031 \\
\hline $\mathrm{H}$ & -6.15711 & 2.55486 & 2.42883 \\
\hline C & -2.69947 & -2.50191 & 0.42135 \\
\hline C & -2.46862 & -3.56025 & -0.49282 \\
\hline C & -2.71163 & -4.87672 & -0.08693 \\
\hline $\mathrm{H}$ & -2.54593 & -5.68905 & -0.79935 \\
\hline C & -3.14420 & -5.17417 & 1.19867 \\
\hline $\mathrm{H}$ & -3.32804 & -6.20948 & 1.49625 \\
\hline C & -3.32063 & -4.13849 & 2.10732 \\
\hline $\mathrm{H}$ & -3.63500 & -4.36999 & 3.12859 \\
\hline C & -3.10244 & -2.80401 & 1.75115 \\
\hline C & -1.96526 & -3.31723 & -1.90155 \\
\hline $\mathrm{H}$ & -1.69333 & -2.25505 & -1.96116 \\
\hline C & -0.70505 & -4.12610 & -2.21141 \\
\hline $\mathrm{H}$ & 0.09879 & -3.92518 & -1.48702 \\
\hline $\mathrm{H}$ & -0.32503 & -3.87080 & -3.21288 \\
\hline $\mathrm{H}$ & -0.89933 & -5.21072 & -2.20510 \\
\hline C & -3.05693 & -3.58342 & -2.93969 \\
\hline $\mathrm{H}$ & -3.35837 & -4.64396 & -2.93612 \\
\hline $\mathrm{H}$ & -2.70022 & -3.33840 & -3.95258 \\
\hline $\mathrm{H}$ & -3.95660 & -2.97879 & -2.74966 \\
\hline C & -3.27859 & -1.74343 & 2.82463 \\
\hline $\mathrm{H}$ & -3.15969 & -0.76102 & 2.34074 \\
\hline $\mathrm{C}$ & -2.18534 & -1.85414 & 3.89015 \\
\hline $\mathrm{H}$ & -2.22987 & -2.82484 & 4.41015 \\
\hline $\mathrm{H}$ & -2.29860 & -1.06014 & 4.64570 \\
\hline $\mathrm{H}$ & -1.18536 & -1.75283 & 3.44149 \\
\hline C & -4.66750 & -1.76885 & 3.46497 \\
\hline $\mathrm{H}$ & -5.46348 & -1.65918 & 2.71421 \\
\hline $\mathrm{H}$ & -4.76942 & -0.94583 & 4.19024 \\
\hline $\mathrm{H}$ & -4.84722 & -2.70815 & 4.01224 \\
\hline
\end{tabular}

(continued)

\begin{tabular}{|c|c|c|c|}
\hline $\mathrm{Al}$ & 1.16436 & 0.16856 & -0.74270 \\
\hline $\mathrm{P}$ & 3.45683 & -0.02680 & 0.57271 \\
\hline $\mathrm{N}$ & 2.47580 & -1.15310 & -0.04802 \\
\hline $\mathrm{C}$ & 3.50029 & -0.04188 & 2.38646 \\
\hline $\mathrm{N}$ & 2.66822 & 1.25135 & -0.03528 \\
\hline $\mathrm{C}$ & 2.27675 & -0.08868 & 3.07468 \\
\hline $\mathrm{H}$ & 1.33210 & -0.10459 & 2.51802 \\
\hline $\mathrm{C}$ & 2.25256 & -0.10817 & 4.46507 \\
\hline $\mathrm{H}$ & 1.29217 & -0.14574 & 4.98527 \\
\hline C & 3.44601 & -0.07897 & 5.18930 \\
\hline $\mathrm{H}$ & 3.42481 & -0.09566 & 6.28193 \\
\hline $\mathrm{C}$ & 4.66383 & -0.02750 & 4.51574 \\
\hline $\mathrm{H}$ & 5.60147 & -0.00362 & 5.07618 \\
\hline C & 4.69305 & -0.01067 & 3.12090 \\
\hline $\mathrm{H}$ & 5.65496 & 0.02276 & 2.60699 \\
\hline $\mathrm{C}$ & 5.20743 & -0.18943 & 0.14512 \\
\hline $\mathrm{C}$ & 5.79347 & -1.46015 & 0.05445 \\
\hline $\mathrm{H}$ & 5.18175 & -2.35839 & 0.15933 \\
\hline $\mathrm{C}$ & 7.16371 & -1.58376 & -0.17049 \\
\hline $\mathrm{H}$ & 7.61038 & -2.57801 & -0.24544 \\
\hline $\mathrm{C}$ & 7.95941 & -0.44564 & -0.29608 \\
\hline $\mathrm{H}$ & 9.03364 & -0.54557 & -0.47040 \\
\hline C & 7.38410 & 0.82049 & -0.19185 \\
\hline $\mathrm{H}$ & 8.00511 & 1.71510 & -0.28138 \\
\hline $\mathrm{C}$ & 6.01454 & 0.95110 & 0.03143 \\
\hline $\mathrm{H}$ & 5.57365 & 1.94660 & 0.12095 \\
\hline C & 2.70051 & -2.50060 & -0.42258 \\
\hline $\mathrm{C}$ & 2.46970 & -3.55989 & 0.49053 \\
\hline $\mathrm{C}$ & 2.71367 & -4.87585 & 0.08360 \\
\hline $\mathrm{H}$ & 2.54784 & -5.68893 & 0.79515 \\
\hline C & 3.14738 & -5.17190 & -1.20194 \\
\hline $\mathrm{H}$ & 3.33203 & -6.20684 & -1.50032 \\
\hline $\mathrm{C}$ & 3.32397 & -4.13530 & -2.10948 \\
\hline $\mathrm{H}$ & 3.63942 & -4.36566 & -3.13069 \\
\hline $\mathrm{C}$ & 3.10471 & -2.80125 & -1.75229 \\
\hline $\mathrm{C}$ & 1.96564 & -3.31837 & 1.89931 \\
\hline $\mathrm{H}$ & 1.69238 & -2.25656 & 1.95948 \\
\hline C & 0.70642 & -4.12906 & 2.20840 \\
\hline $\mathrm{H}$ & 0.90221 & -5.21340 & 2.20207 \\
\hline $\mathrm{H}$ & 0.32553 & -3.87444 & 3.20971 \\
\hline $\mathrm{H}$ & -0.09736 & -3.92916 & 1.48364 \\
\hline $\mathrm{C}$ & 3.05737 & -3.58385 & 2.93757 \\
\hline $\mathrm{H}$ & 3.95609 & -2.97743 & 2.74867 \\
\hline $\mathrm{H}$ & 2.69982 & -3.34062 & 3.95060 \\
\hline $\mathrm{H}$ & 3.36063 & -4.64386 & 2.93288 \\
\hline C & 3.28109 & -1.73969 & -2.82477 \\
\hline $\mathrm{H}$ & 3.16073 & -0.75775 & -2.34026 \\
\hline C & 2.18917 & -1.85052 & -3.89161 \\
\hline $\mathrm{H}$ & 1.18857 & -1.75050 & -3.44402 \\
\hline $\mathrm{H}$ & 2.30260 & -1.05580 & -4.64637 \\
\hline $\mathrm{H}$ & 2.23513 & -2.82076 & -4.41235 \\
\hline $\mathrm{C}$ & 4.67075 & -1.76346 & -3.46351 \\
\hline $\mathrm{H}$ & 4.85167 & -2.70204 & -4.01163 \\
\hline $\mathrm{H}$ & 4.77303 & -0.93956 & -4.18773 \\
\hline $\mathrm{H}$ & 5.46579 & -1.65420 & -2.71170 \\
\hline $\mathrm{C}$ & 2.99155 & 2.62728 & -0.14979 \\
\hline $\mathrm{C}$ & 2.59053 & 3.56192 & 0.83529 \\
\hline $\mathrm{C}$ & 2.91067 & 4.91366 & 0.66008 \\
\hline $\mathrm{H}$ & 2.60943 & 5.63116 & 1.42783 \\
\hline $\mathrm{C}$ & 3.58918 & 5.36264 & -0.46284 \\
\hline $\mathrm{H}$ & 3.82998 & 6.42229 & -0.57869 \\
\hline C & 3.94446 & 4.44866 & -1.44837 \\
\hline $\mathrm{H}$ & 4.45629 & 4.80372 & -2.34543 \\
\hline C & 3.65427 & 3.08774 & -1.32304 \\
\hline $\mathrm{C}$ & 1.80669 & 3.16911 & 2.07228 \\
\hline $\mathrm{H}$ & 1.56382 & 2.10169 & 1.97965 \\
\hline $\mathrm{C}$ & 0.47868 & 3.92272 & 2.16541 \\
\hline $\mathrm{H}$ & -0.14421 & 3.76595 & 1.27162 \\
\hline $\mathrm{H}$ & -0.09688 & 3.57462 & 3.03657 \\
\hline $\mathrm{H}$ & 0.62833 & 5.00809 & 2.28246 \\
\hline C & 2.63244 & 3.36136 & 3.34528 \\
\hline $\mathrm{H}$ & 2.89622 & 4.42151 & 3.49320 \\
\hline $\mathrm{H}$ & 2.06440 & 3.03256 & 4.22948 \\
\hline $\mathrm{H}$ & 3.56823 & 2.78342 & 3.31472 \\
\hline $\mathrm{C}$ & 4.00042 & 2.15738 & -2.47403 \\
\hline $\mathrm{H}$ & 4.08545 & 1.14156 & -2.06161 \\
\hline C & 2.86970 & 2.13687 & -3.50654 \\
\hline $\mathrm{H}$ & 2.70570 & 3.14297 & -3.92553 \\
\hline $\mathrm{H}$ & 3.11213 & 1.45647 & -4.33900 \\
\hline $\mathrm{H}$ & 1.92120 & 1.79685 & -3.05931 \\
\hline $\mathrm{C}$ & 5.33031 & 2.48182 & -3.15354 \\
\hline $\mathrm{H}$ & 6.15515 & 2.56216 & -2.43020 \\
\hline $\mathrm{H}$ & 5.58955 & 1.68818 & -3.87138 \\
\hline $\mathrm{H}$ & 5.28599 & 3.42461 & -3.72150 \\
\hline
\end{tabular}


[ $\left({ }^{\text {Dip }} \mathbf{L A l}\right)_{2}$ ], singlet, pbe0-gd3-bj/def2-tzvp 168

$\begin{array}{llrr}\mathrm{Al} & -1.11095 & 0.19615 & 0.74814 \\ \mathrm{P} & -3.33224 & -0.02719 & -0.64243 \\ \mathrm{~N} & -2.60605 & 1.24925 & 0.02935 \\ \mathrm{C} & -3.32181 & 0.00012 & -2.44586 \\ \mathrm{~N} & -2.33916 & -1.13889 & -0.02477 \\ \mathrm{C} & -2.07991 & -0.06576 & -3.09649 \\ \mathrm{H} & -1.15464 & -0.11183 & -2.50940 \\ \mathrm{C} & -2.01669 & -0.05809 & -4.48449\end{array}$

$\begin{array}{llll}\mathrm{H} & -1.04292 & -0.11062 & -4.97763 \\ \mathrm{C} & -3.18869 & 0.01641 & -5.23956\end{array}$

$\begin{array}{llll}\mathrm{C} & -3.18869 & 0.01641 & -5.23956 \\ \mathrm{H} & -3.13773 & 0.01898 & -6.33113\end{array}$

$\begin{array}{llll}\mathrm{H} & -3.13773 & 0.01898 & -6.33113 \\ \mathrm{C} & -4.42432 & 0.08886 & -4.60031 \\ \mathrm{H} & -5.34349 & 0.14905 & -5.18749 \\ \mathrm{C} & -4.49371 & 0.07956 & -3.20750\end{array}$

$\begin{array}{llll}\mathrm{C} & -4.49371 & 0.07956 & -3.20750\end{array}$

$\begin{array}{rrrr}\mathrm{H} & -5.46631 & 0.12763 & -2.71507 \\ \mathrm{C} & -5.07395 & -0.27082 & -0.25451\end{array}$

$\begin{array}{llll}\mathrm{C} & -5.07395 & -0.27082 & -0.25451 \\ \mathrm{C} & -5.90963 & 0.83044 & -0.03252\end{array}$

$\begin{array}{llll}\mathrm{C} & -5.90963 & 0.83044 & -0.03252 \\ \mathrm{H} & -5.49799 & 1.84204 & -0.05134 \\ \mathrm{C} & -7.26772 & 0.63659 & 0.20960\end{array}$

$\begin{array}{llll}\mathrm{C} & -7.26772 & 0.63659 & 0.20960 \\ \mathrm{H} & -7.91444 & 1.49880 & 0.38770\end{array}$

$\begin{array}{rrrr}\mathrm{H} & -7.91444 & 1.49880 & 0.38770 \\ \mathrm{C} & -7.79867 & -0.65294 & 0.22181\end{array}$

$\begin{array}{llll}\mathrm{C} & -7.79867 & -0.65294 & 0.22181 \\ \mathrm{H} & -8.86365 & -0.80278 & 0.41479\end{array}$

$\begin{array}{lllr}\mathrm{H} & -8.86365 & -0.80278 & 0.41479 \\ \mathrm{C} & -6.97311 & -1.75057 & -0.02039\end{array}$

$\begin{array}{llll}\mathrm{C} & -6.97311 & -1.75057 & -0.02039 \\ \mathrm{H} & -7.38701 & -2.76134 & -0.01822 \\ \mathrm{C} & -5.61437 & -1.56413 & -0.26412\end{array}$

$\begin{array}{llll}\mathrm{C} & -5.61437 & -1.56413 & -0.26412 \\ \mathrm{H} & -4.97547 & -2.42829 & -0.45480\end{array}$

$\begin{array}{llll}\mathrm{H} & -4.97547 & -2.42829 & -0.45480 \\ \mathrm{C} & -2.90813 & 2.62396 & 0.13189\end{array}$

$\begin{array}{lrrr}\mathrm{C} & -2.90813 & 2.62396 & 0.13189 \\ \mathrm{C} & -2.42412 & 3.55108 & -0.81776\end{array}$

$\begin{array}{llll}\mathrm{C} & -2.42412 & 3.55108 & -0.81776 \\ \mathrm{C} & -2.70488 & 4.91003 & -0.63775\end{array}$

$\begin{array}{llll}\mathrm{C} & -2.70488 & 4.91003 & -0.63775 \\ \mathrm{H} & -2.33774 & 5.62766 & -1.37571 \\ \mathrm{C} & -3.42467 & 5.36378 & 0.45799\end{array}$

$\begin{array}{llll}\mathrm{C} & -3.42467 & 5.36378 & 0.45799 \\ \mathrm{H} & -3.63299 & 6.42941 & 0.57969\end{array}$

$\begin{array}{llll}\mathrm{H} & -3.63299 & 6.42941 & 0.57969 \\ \mathrm{C} & -3.86427 & 4.45014 & 1.41040\end{array}$

$\begin{array}{llll}\mathrm{C} & -3.86427 & 4.45014 & 1.41040 \\ \mathrm{H} & -4.40800 & 4.81006 & 2.28625 \\ \mathrm{C} & -3.61308 & 3.08331 & 1.27628\end{array}$

$\begin{array}{lllr}\mathrm{C} & -3.61308 & 3.08331 & 1.27628 \\ \mathrm{C} & -1.59423 & 3.13055 & -2.01209\end{array}$

$\begin{array}{llll}\mathrm{C} & -1.59423 & 3.13055 & -2.01209 \\ \mathrm{H} & -1.37413 & 2.06025 & -1.89524 \\ \mathrm{C} & -0.24944 & 3.85445 & -2.05777\end{array}$

$\begin{array}{llll}\mathrm{C} & -0.24944 & 3.85445 & -2.05777 \\ \mathrm{H} & -0.36843 & 4.94201 & -2.18593\end{array}$

$\begin{array}{rrrr}\mathrm{H} & -0.36843 & 4.94201 & -2.18593 \\ \mathrm{H} & 0.35047 & 3.48658 & -2.90293 \\ \mathrm{H} & 0.33615 & 3.68953 & -1.14137\end{array}$

$\begin{array}{lrrr}\mathrm{H} & 0.33615 & 3.68953 & -1.14137 \\ \mathrm{C} & -2.36606 & 3.31894 & -3.31706\end{array}$

$\begin{array}{llll}\mathrm{C} & -2.36606 & 3.31894 & -3.31706 \\ \mathrm{H} & -3.31250 & 2.75842 & -3.31147 \\ \mathrm{H} & -1.77179 & 2.96534 & -4.17351\end{array}$

$\begin{array}{llll}\mathrm{H} & -1.77179 & 2.96534 & -4.17351 \\ \mathrm{H} & -2.60382 & 4.38179 & -3.48693\end{array}$

$\begin{array}{llll}\mathrm{H} & -2.60382 & 4.38179 & -3.48693 \\ \mathrm{C} & -4.02939 & 2.13429 & 2.38541 \\ \mathrm{H} & -4.14489 & 1.13694 & 1.93896\end{array}$

$\begin{array}{llll}\mathrm{H} & -4.14489 & 1.13694 & 1.93896 \\ \mathrm{C} & -2.92788 & 2.02835 & 3.44112\end{array}$

$\begin{array}{llll}\mathrm{C} & -2.92788 & 2.02835 & 3.44112 \\ \mathrm{H} & -1.98796 & 1.65011 & 3.00475 \\ \mathrm{H} & -3.22492 & 1.33919 & 4.24751\end{array}$

$\begin{array}{llll}\mathrm{H} & -3.22492 & 1.33919 & 4.24751 \\ \mathrm{H} & -2.72078 & 3.01286 & 3.89002\end{array}$

$\begin{array}{llll}\mathrm{H} & -2.72078 & 3.01286 & 3.89002 \\ \mathrm{C} & -5.36129 & 2.49483 & 3.03697\end{array}$

$\begin{array}{llll}\mathrm{H} & -5.29098 & 3.41391 & 3.63969 \\ \mathrm{H} & -5.67955 & 1.68790 & 3.71467\end{array}$

$\begin{array}{llll}\mathrm{H} & -5.67955 & 1.68790 & 3.71467 \\ \mathrm{H} & -6.15708 & 2.64159 & 2.29184\end{array}$

$\begin{array}{llll}\text { C } & -2.57502 & -2.47672 & 0.35266\end{array}$

$\begin{array}{llll}\mathrm{C} & -2.28999 & -3.53410 & -0.54145 \\ \mathrm{C} & -2.55608 & -4.84752 & -0.14331\end{array}$

$\begin{array}{llll}\mathrm{C} & -2.55608 & -4.84752 & -0.14331 \\ \mathrm{H} & -2.34730 & -5.66533 & -0.83728\end{array}$

$\begin{array}{llll}\mathrm{H} & -2.34730 & -5.66533 & -0.83728 \\ \mathrm{C} & -3.06722 & -5.13181 & 1.11751\end{array}$

$\begin{array}{llll}\mathrm{C} & -3.06722 & -5.13181 & 1.11751 \\ \mathrm{H} & -3.26893 & -6.16467 & 1.41112\end{array}$

$\begin{array}{llll}\mathrm{H} & -3.26893 & -6.16467 & 1.41112 \\ \mathrm{C} & -3.29751 & -4.09119 & 2.00959 \\ \mathrm{H} & -3.67185 & -4.31534 & 3.01193\end{array}$

$\begin{array}{llll}\mathrm{H} & -3.67185 & -4.31534 & 3.01193 \\ \mathrm{C} & -3.05439 & -2.76123 & 1.65641\end{array}$

\begin{tabular}{lllr}
$\mathrm{C}$ & -3.05439 & -2.76123 & 1.65641 \\
$\mathrm{C}$ & -1.72012 & -3.26936 & -1.91805 \\
\hline
\end{tabular}

$\begin{array}{llll}\mathrm{H} & -1.39635 & -3.26936 & -1.91805 \\ \mathrm{C} & -0.49127 & -4.12544 & -2.92343 \\ \mathrm{H} & & 0.28966 & -3.98823\end{array}$

$\begin{array}{llll}\mathrm{C} & -0.49127 & -4.12544 & -2.21301 \\ \mathrm{H} & 0.28966 & -3.98823 & -1.45109\end{array}$

$\begin{array}{llll}\mathrm{H} & 0.28966 & -3.98823 & -1.45109 \\ \mathrm{H} & -0.06015 & -3.85060 & -3.18755 \\ \mathrm{H} & -0.73543 & -5.19882 & -2.25690\end{array}$

$\begin{array}{llll}\mathrm{H} & -0.06015 & -3.85060 & -3.18755 \\ \mathrm{C} & -0.73543 & -5.19882 & -2.25690 \\ \mathrm{C} & -2.78993 & -3.44008 & -2.99638\end{array}$

$\begin{array}{llll}\mathrm{C} & -2.78993 & -3.44008 & -2.99638 \\ \mathrm{H} & -3.13986 & -4.48451 & -3.04097\end{array}$

$\begin{array}{llll}\mathrm{H} & -2.39351 & -3.17045 & -3.98768\end{array}$

$\begin{array}{llll}\mathrm{H} & -3.66441 & -2.80004 & -2.80616 \\ \mathrm{C} & -3.27262 & -1.67823 & 2.69558\end{array}$

$\begin{array}{llll}\mathrm{C} & -3.27262 & -1.67823 & 2.69558 \\ \mathrm{H} & -3.12595 & -0.70812 & 2.19505\end{array}$

$\begin{array}{llll}\mathrm{C} & -2.22194 & -1.77363 & 3.80271\end{array}$

$\begin{array}{llll}\mathrm{H} & -2.28697 & -2.73720 & 4.33296\end{array}$

$\begin{array}{llll}\mathrm{H} & -2.35970 & -0.96790 & 4.54071 \\ \mathrm{H} & -1.20702 & -1.68035 & 3.38660\end{array}$

$\begin{array}{llll}\mathrm{H} & -1.20702 & -1.68035 & 3.38660 \\ \mathrm{C} & -4.68804 & -1.67412 & 3.26920\end{array}$

$\begin{array}{llll}\mathrm{H} & -5.44252 & -1.56505 & 2.47715 \\ \mathrm{H} & -4.81146 & -0.83710 & 3.97435\end{array}$

$\begin{array}{llll}\mathrm{H} & -4.81146 & -0.83710 & 3.97435 \\ \mathrm{H} & -4.90768 & -2.60210 & 3.82077\end{array}$

$\begin{array}{lll}-4.90768 & -2.60210 & 3.82077\end{array}$


[ ${ }^{\text {Dip }}$ LGa:], singlet, pbe0/def2-tzvp 84

\begin{tabular}{|c|c|c|c|}
\hline $\mathrm{Ga}$ & -0.00001 & -0.31878 & -2.41069 \\
\hline $\mathrm{P}$ & 0.00000 & 0.30434 & 0.28711 \\
\hline $\mathrm{N}$ & -1.22241 & -0.01887 & -0.70967 \\
\hline $\mathrm{C}$ & -0.00008 & 2.04338 & 0.83282 \\
\hline $\mathrm{C}$ & -0.00010 & 3.04614 & -0.14937 \\
\hline $\mathrm{H}$ & -0.00006 & 2.76892 & -1.20745 \\
\hline $\mathrm{C}$ & -0.00016 & 4.39044 & 0.20450 \\
\hline $\mathrm{H}$ & -0.00017 & 5.15758 & -0.57359 \\
\hline $\mathrm{C}$ & -0.00019 & 4.75726 & 1.55224 \\
\hline $\mathrm{H}$ & -0.00023 & 5.81353 & 1.83228 \\
\hline C & -0.00017 & 3.77305 & 2.53632 \\
\hline $\mathrm{H}$ & -0.00019 & 4.05299 & 3.59251 \\
\hline $\mathrm{C}$ & -0.00011 & 2.42306 & 2.18021 \\
\hline $\mathrm{H}$ & -0.00009 & 1.66340 & 2.96343 \\
\hline $\mathrm{C}$ & 0.00002 & -0.59287 & 1.86065 \\
\hline $\mathrm{C}$ & -1.21067 & -0.89197 & 2.50141 \\
\hline $\mathrm{H}$ & -2.16097 & -0.64688 & 2.02261 \\
\hline $\mathrm{C}$ & -1.20725 & -1.50159 & 3.75508 \\
\hline $\mathrm{H}$ & -2.15566 & -1.73402 & 4.24545 \\
\hline $\mathrm{C}$ & 0.00005 & -1.80929 & 4.38157 \\
\hline $\mathrm{H}$ & 0.00005 & -2.28580 & 5.36509 \\
\hline $\mathrm{C}$ & -2.59148 & -0.29910 & -0.51003 \\
\hline C & -3.01111 & -1.64996 & -0.34669 \\
\hline $\mathrm{C}$ & -4.36865 & -1.92512 & -0.16477 \\
\hline $\mathrm{H}$ & -4.68832 & -2.96186 & -0.03480 \\
\hline $\mathrm{C}$ & -5.32223 & -0.91342 & -0.15398 \\
\hline $\mathrm{H}$ & -6.37841 & -1.14962 & -0.00325 \\
\hline C & -4.91505 & 0.39737 & -0.35899 \\
\hline $\mathrm{H}$ & -5.66275 & 1.19507 & -0.38027 \\
\hline $\mathrm{C}$ & -3.56908 & 0.72640 & -0.55213 \\
\hline $\mathrm{C}$ & -2.03456 & -2.80902 & -0.43539 \\
\hline $\mathrm{H}$ & -1.02815 & -2.39939 & -0.26428 \\
\hline $\mathrm{C}$ & -2.05499 & -3.40965 & -1.84462 \\
\hline $\mathrm{H}$ & -1.32154 & -4.22691 & -1.93692 \\
\hline $\mathrm{H}$ & -1.82007 & -2.65575 & -2.61272 \\
\hline $\mathrm{H}$ & -3.05087 & -3.81855 & -2.08019 \\
\hline C & -2.26385 & -3.89461 & 0.61490 \\
\hline $\mathrm{H}$ & -2.27610 & -3.47951 & 1.63343 \\
\hline $\mathrm{H}$ & -1.45809 & -4.64391 & 0.56633 \\
\hline $\mathrm{H}$ & -3.21173 & -4.43269 & 0.45555 \\
\hline $\mathrm{C}$ & -3.21412 & 2.17030 & -0.84274 \\
\hline $\mathrm{H}$ & -2.12716 & 2.20449 & -0.97965 \\
\hline $\mathrm{C}$ & -3.84831 & 2.64837 & -2.15086 \\
\hline $\mathrm{H}$ & -3.56646 & 1.99744 & -2.99257 \\
\hline $\mathrm{H}$ & -3.52149 & 3.67377 & -2.38808 \\
\hline $\mathrm{H}$ & -4.94873 & 2.65834 & -2.09207 \\
\hline $\mathrm{C}$ & -3.56319 & 3.10002 & 0.31901 \\
\hline $\mathrm{H}$ & -4.64828 & 3.11734 & 0.51188 \\
\hline $\mathrm{H}$ & -3.24838 & 4.13256 & 0.09979 \\
\hline $\mathrm{H}$ & -3.06183 & 2.78793 & 1.24778 \\
\hline $\mathrm{N}$ & 1.22242 & -0.01877 & -0.70968 \\
\hline C & 1.21073 & -0.89228 & 2.50124 \\
\hline $\mathrm{H}$ & 2.16103 & -0.64743 & 2.02230 \\
\hline $\mathrm{C}$ & 1.20734 & -1.50189 & 3.75491 \\
\hline $\mathrm{H}$ & 2.15575 & -1.73456 & 4.24515 \\
\hline $\mathrm{C}$ & 2.59151 & -0.29896 & -0.51008 \\
\hline $\mathrm{C}$ & 3.01120 & -1.64983 & -0.34696 \\
\hline $\mathrm{C}$ & 4.36875 & -1.92495 & -0.16504 \\
\hline $\mathrm{H}$ & 4.68847 & -2.96170 & -0.03525 \\
\hline $\mathrm{C}$ & 5.32228 & -0.91322 & -0.15405 \\
\hline $\mathrm{H}$ & 6.37847 & -1.14938 & -0.00334 \\
\hline $\mathrm{C}$ & 4.91504 & 0.39759 & -0.35885 \\
\hline $\mathrm{H}$ & 5.66271 & 1.19534 & -0.37997 \\
\hline $\mathrm{C}$ & 3.56906 & 0.72659 & -0.55197 \\
\hline $\mathrm{C}$ & 2.03472 & -2.80893 & -0.43586 \\
\hline $\mathrm{H}$ & 1.02826 & -2.39935 & -0.26490 \\
\hline $\mathrm{C}$ & 2.05542 & -3.40952 & -1.84511 \\
\hline $\mathrm{H}$ & 1.32201 & -4.22680 & -1.93755 \\
\hline $\mathrm{H}$ & 1.82061 & -2.65561 & -2.61322 \\
\hline $\mathrm{H}$ & 3.05135 & -3.81839 & -2.08050 \\
\hline $\mathrm{C}$ & 2.26390 & -3.89453 & 0.61444 \\
\hline $\mathrm{H}$ & 2.27594 & -3.47946 & 1.63299 \\
\hline $\mathrm{H}$ & 1.45820 & -4.64390 & 0.56571 \\
\hline $\mathrm{H}$ & 3.21185 & -4.43254 & 0.45526 \\
\hline $\mathrm{C}$ & 3.21404 & 2.17052 & -0.84234 \\
\hline $\mathrm{H}$ & 2.12708 & 2.20468 & -0.97928 \\
\hline $\mathrm{C}$ & 3.84825 & 2.64887 & -2.15035 \\
\hline $\mathrm{H}$ & 3.56648 & 1.99806 & -2.99218 \\
\hline $\mathrm{H}$ & 3.52138 & 3.67429 & -2.38740 \\
\hline $\mathrm{H}$ & 4.94867 & 2.65889 & -2.09151 \\
\hline $\mathrm{C}$ & 3.56301 & 3.10006 & 0.31960 \\
\hline $\mathrm{H}$ & 4.64809 & 3.11742 & 0.51249 \\
\hline $\mathrm{H}$ & 3.24813 & 4.13262 & 0.10055 \\
\hline $\mathrm{H}$ & 3.06165 & 2.78776 & 1.24829 \\
\hline
\end{tabular}

[ ${ }^{\text {Dip }}$ LGa:], singlet, pbe0-gd3-bj/def2-tzvp 84

$\begin{array}{llll}\mathrm{Ga} & -0.00000 & -0.30823 & -2.47196 \\ \mathrm{P} & -0.00001 & 0.31143 & 0.23001 \\ \mathrm{~N} & -1.22053 & -0.01039 & -0.76486 \\ \mathrm{C} & -0.00044 & 2.04397 & 0.76419 \\ \mathrm{C} & -0.00052 & 3.03580 & -0.22738 \\ \mathrm{H} & -0.00032 & 2.74707 & -1.28228 \\ \mathrm{C} & -0.00085 & 4.38133 & 0.11722 \\ \mathrm{H} & -0.00091 & 5.14339 & -0.66548 \\ \mathrm{C} & -0.00111 & 4.75654 & 1.46289 \\ \mathrm{H} & -0.00137 & 5.81460 & 1.73513 \\ \mathrm{C} & -0.00103 & 3.78044 & 2.45493 \\ \mathrm{H} & -0.00122 & 4.06896 & 3.50850 \\ \mathrm{C} & -0.00069 & 2.42847 & 2.10875 \\ \mathrm{H} & -0.00061 & 1.67046 & 2.89357 \\ \mathrm{C} & 0.00020 & -0.58496 & 1.79564 \\ \mathrm{C} & -1.21130 & -0.88854 & 2.43172 \\ \mathrm{H} & -2.16041 & -0.63398 & 1.95673 \\ \mathrm{C} & -1.20718 & -1.51818 & 3.67450 \\ \mathrm{H} & -2.15541 & -1.75753 & 4.16134 \\ \mathrm{C} & 0.00044 & -1.83853 & 4.29386\end{array}$

$\begin{array}{lll}0.00053 & -2.33331 & 5.26807\end{array}$

$\begin{array}{lll}-2.57616 & -0.30293 & -0.52050\end{array}$

$\begin{array}{lll}-2.96943 & -1.65518 & -0.32860\end{array}$

$\begin{array}{lll}-4.31381 & -1.94512 & -0.09009\end{array}$

$\begin{array}{lll}-4.61589 & -2.98364 & 0.06303\end{array}$

$\begin{array}{lll}-5.27549 & -0.94093 & -0.04614\end{array}$

$\begin{array}{lll}-6.32191 & -1.18689 & 0.14909\end{array}$

$\begin{array}{lll}-4.89183 & 0.37445 & -0.26862\end{array}$

$\begin{array}{lll}-5.64658 & 1.16537 & -0.25490\end{array}$

$\begin{array}{lll}-3.55822 & 0.71442 & -0.51772\end{array}$

$\begin{array}{lll}-1.96751 & -2.78704 & -0.43328\end{array}$

$\begin{array}{lll}-0.97294 & -2.35157 & -0.26179\end{array}$

$\begin{array}{lll}-1.97714 & -3.36752 & -1.84874\end{array}$

$\begin{array}{lll}-1.22375 & -4.16389 & -1.95537\end{array}$

$\begin{array}{lll}-1.76295 & -2.59520 & -2.60455\end{array}$

$\begin{array}{lll}-2.96409 & -3.79514 & -2.08721\end{array}$

$\begin{array}{lll}-2.16055 & -3.88546 & 0.60747\end{array}$

$\begin{array}{lll}-2.17351 & -3.47644 & 1.62821\end{array}$

$\begin{array}{lll}-1.33556 & -4.61215 & 0.54632\end{array}$

$\begin{array}{lll}-3.09573 & -4.44590 & 0.45138\end{array}$

$\begin{array}{lll}-3.20791 & 2.16030 & -0.79092\end{array}$

$\begin{array}{lll}-2.13936 & 2.17976 & -1.03561\end{array}$

$\begin{array}{lll}-3.95938 & 2.70574 & -2.00428\end{array}$

$\begin{array}{lll}-3.78356 & 2.08226 & -2.89389\end{array}$

$\begin{array}{lll}-3.62896 & 3.73137 & -2.23389\end{array}$

$\begin{array}{lll}-5.04685 & 2.74119 & -1.83094\end{array}$

$\begin{array}{lll}-3.41888 & 3.03754 & 0.44127\end{array}$

$\begin{array}{lll}-4.47940 & 3.05879 & 0.7403\end{array}$

$\begin{array}{lll}-3.10382 & 4.07378 & 0.24263\end{array}$

$\begin{array}{lll}-2.83476 & 2.66972 & 1.29789\end{array}$

$\begin{array}{lll}1.22059 & -0.00984 & -0.76493\end{array}$

$\begin{array}{lll}1.21182 & -0.88887 & 2.43131\end{array}$

$\begin{array}{lll}2.16084 & -0.63457 & 1.95601\end{array}$

$\begin{array}{lll}2.16084 & -1.51851 & 3.67410\end{array}$

$\begin{array}{lll}2.15626 & -1.75813 & 4.16063\end{array}$

$\begin{array}{lll}2.57630 & -0.30217 & -0.52071\end{array}$

$2.96991 \quad-1.65446 \quad-0.32976$

$4.31435 \quad-1.94420 \quad-0.09134$

$\begin{array}{lll}4.61669 & -2.98276 & 0.06105\end{array}$

$\begin{array}{lll}5.27576 & -0.93978 & -0.04656\end{array}$

$\begin{array}{lll}6.32223 & -1.18560 & 0.1486\end{array}$

$\begin{array}{lll}4.89177 & 0.37565 & -0.26812\end{array}$

$\begin{array}{lll}5.64630 & 1.16676 & -0.25375\end{array}$

$\begin{array}{lll}3.55809 & 0.71544 & -0.51712\end{array}$

$\begin{array}{lll}1.96836 & -2.78655 & -0.43534\end{array}$

$\begin{array}{lll}0.97357 & -2.35139 & -0.26437\end{array}$

$\begin{array}{lll}0.97906 & -3.36669 & -1.85094\end{array}$

$1.22594 \quad-4.16324 \quad-1.95825$

$\begin{array}{lll}1.76513 & -2.59424 & -2.60668\end{array}$

$\begin{array}{lll}2.96627 & -3.79399 & -2.08888\end{array}$

$\begin{array}{lll}2.16107 & -3.88515 & 0.60528\end{array}$

$\begin{array}{lll}2.17312 & -3.47638 & 1.62613\end{array}$

$\begin{array}{lll}1.33640 & -4.61216 & 0.54336 \\ 3.09658 & -4.44519 & 0.44973\end{array}$

$\begin{array}{lll}3.09658 & -4.44519 & 0.44973\end{array}$

$\begin{array}{lll}3.20739 & 2.16142 & -0.78929\end{array}$

$\begin{array}{lll}2.13891 & 2.18072 & -1.03427\end{array}$

$\begin{array}{lll}2.95904 & 2.70809 & -2.00197\end{array}$

$\begin{array}{lll}3.78374 & 2.08526 & -2.89214 \\ 3.62831 & 3.73379 & -2.23087\end{array}$

$\begin{array}{lll}3.62831 & 3.73379 & -2.23087\end{array}$

$\begin{array}{lll}5.04644 & 2.74383 & -1.82826\end{array}$

$\begin{array}{lll}3.41769 & 3.03773 & 0.44367\end{array}$

$\begin{array}{lll}4.47813 & 3.05918 & 0.74298\end{array}$

$\begin{array}{lll}3.10224 & 4.07400 & 0.24582\end{array}$

$\begin{array}{lll}3.10224 & 4.07400 & 0.24582 \\ 2.83354 & 2.66896 & 1.29986\end{array}$ 
[ ${ }^{\text {Dip }}$ LGa:], triplet, pbe0/def2-tzvp 84

\begin{tabular}{|c|c|c|c|}
\hline $\mathrm{Ga}$ & 0.15859 & -0.65021 & -2.11141 \\
\hline $\mathrm{P}$ & -0.04219 & 0.47535 & 0.30509 \\
\hline $\mathrm{N}$ & -1.15358 & -0.21216 & -0.82998 \\
\hline $\mathrm{C}$ & -0.42650 & 2.15457 & 0.57149 \\
\hline $\mathrm{C}$ & -0.48702 & 3.02306 & -0.56300 \\
\hline $\mathrm{H}$ & -0.36864 & 2.61183 & -1.56916 \\
\hline $\mathrm{C}$ & -0.67712 & 4.38920 & -0.41022 \\
\hline $\mathrm{H}$ & -0.71547 & 5.02354 & -1.30048 \\
\hline $\mathrm{C}$ & -0.83023 & 4.95795 & 0.85796 \\
\hline $\mathrm{H}$ & -0.99326 & 6.03139 & 0.97184 \\
\hline $\mathrm{C}$ & -0.76107 & 4.11806 & 1.98656 \\
\hline $\mathrm{H}$ & -0.86180 & 4.54451 & 2.98826 \\
\hline $\mathrm{C}$ & -0.55088 & 2.75861 & 1.85692 \\
\hline $\mathrm{H}$ & -0.46661 & 2.14466 & 2.75457 \\
\hline $\mathrm{C}$ & -0.03167 & -0.39789 & 1.85153 \\
\hline $\mathrm{C}$ & -1.15497 & -0.31011 & 2.70479 \\
\hline $\mathrm{H}$ & -2.02970 & 0.26528 & 2.39454 \\
\hline $\mathrm{C}$ & -1.16130 & -0.96497 & 3.93199 \\
\hline $\mathrm{H}$ & -2.03657 & -0.87867 & 4.58092 \\
\hline $\mathrm{C}$ & -0.07230 & -1.74648 & 4.32503 \\
\hline $\mathrm{H}$ & -0.08465 & -2.26545 & 5.28620 \\
\hline $\mathrm{C}$ & -2.45994 & -0.64926 & -0.53390 \\
\hline $\mathrm{C}$ & -2.65936 & -1.97622 & -0.05415 \\
\hline $\mathrm{C}$ & -3.94973 & -2.39154 & 0.27366 \\
\hline $\mathrm{H}$ & -4.10857 & -3.40525 & 0.64733 \\
\hline $\mathrm{C}$ & -5.04093 & -1.53838 & 0.13435 \\
\hline $\mathrm{H}$ & -6.04336 & -1.87917 & 0.40525 \\
\hline $\mathrm{C}$ & -4.84751 & -0.25891 & -0.36694 \\
\hline $\mathrm{H}$ & -5.70817 & 0.40197 & -0.49949 \\
\hline $\mathrm{C}$ & -3.57531 & 0.20584 & -0.71875 \\
\hline $\mathrm{C}$ & -1.51218 & -2.96684 & 0.02275 \\
\hline $\mathrm{H}$ & -0.59137 & -2.38415 & 0.17004 \\
\hline $\mathrm{C}$ & -1.38461 & -3.72150 & -1.30409 \\
\hline $\mathrm{H}$ & -0.51362 & -4.39682 & -1.29859 \\
\hline $\mathrm{H}$ & -1.27788 & -3.03517 & -2.16346 \\
\hline $\mathrm{H}$ & -2.28430 & -4.32680 & -1.49832 \\
\hline $\mathrm{C}$ & -1.59724 & -3.93742 & 1.19700 \\
\hline $\mathrm{H}$ & -1.70589 & -3.39925 & 2.15000 \\
\hline $\mathrm{H}$ & -0.67809 & -4.54104 & 1.25423 \\
\hline $\mathrm{H}$ & -2.43910 & -4.64125 & 1.09868 \\
\hline C & -3.45077 & 1.58162 & -1.33900 \\
\hline $\mathrm{H}$ & -2.38176 & 1.76851 & -1.49637 \\
\hline $\mathrm{C}$ & -4.13236 & 1.62325 & -2.70946 \\
\hline $\mathrm{H}$ & -3.73606 & 0.84554 & -3.38095 \\
\hline $\mathrm{H}$ & -3.97410 & 2.60181 & -3.19059 \\
\hline $\mathrm{H}$ & -5.22023 & 1.46622 & -2.62789 \\
\hline $\mathrm{C}$ & -3.97247 & 2.68614 & -0.42105 \\
\hline $\mathrm{H}$ & -5.04990 & 2.57370 & -0.21701 \\
\hline $\mathrm{H}$ & -3.82239 & 3.67370 & -0.88376 \\
\hline $\mathrm{H}$ & -3.43836 & 2.68947 & 0.54079 \\
\hline $\mathrm{N}$ & 1.25304 & 0.09281 & -0.72199 \\
\hline $\mathrm{C}$ & 1.05613 & -1.20008 & 2.25288 \\
\hline $\mathrm{H}$ & 1.93955 & -1.27542 & 1.61848 \\
\hline $\mathrm{C}$ & 1.03081 & -1.86402 & 3.47359 \\
\hline $\mathrm{H}$ & 1.89074 & -2.46904 & 3.77253 \\
\hline $\mathrm{C}$ & 2.64306 & 0.00370 & -0.48127 \\
\hline $\mathrm{C}$ & 3.26930 & -1.27640 & -0.55425 \\
\hline $\mathrm{C}$ & 4.64306 & -1.37730 & -0.32622 \\
\hline $\mathrm{H}$ & 5.12402 & -2.35647 & -0.38804 \\
\hline $\mathrm{C}$ & 5.41286 & -0.26162 & -0.01732 \\
\hline $\mathrm{H}$ & 6.48416 & -0.36410 & 0.17137 \\
\hline $\mathrm{C}$ & 4.80358 & 0.98303 & 0.03347 \\
\hline $\mathrm{H}$ & 5.40727 & 1.86708 & 0.25489 \\
\hline $\mathrm{C}$ & 3.43534 & 1.14682 & -0.21036 \\
\hline $\mathrm{C}$ & 2.51429 & -2.54098 & -0.93070 \\
\hline $\mathrm{H}$ & 1.43813 & -2.34081 & -0.78152 \\
\hline $\mathrm{C}$ & 2.73868 & -2.88336 & -2.40721 \\
\hline $\mathrm{H}$ & 2.13768 & -3.75525 & -2.71197 \\
\hline $\mathrm{H}$ & 2.47788 & -2.04141 & -3.07059 \\
\hline $\mathrm{H}$ & 3.79880 & -3.11767 & -2.59250 \\
\hline $\mathrm{C}$ & 2.83989 & -3.74391 & -0.04500 \\
\hline $\mathrm{H}$ & 2.68638 & -3.51748 & 1.02030 \\
\hline $\mathrm{H}$ & 2.19154 & -4.59504 & -0.30625 \\
\hline $\mathrm{H}$ & 3.88046 & -4.08003 & -0.17317 \\
\hline $\mathrm{C}$ & 2.87552 & 2.55313 & -0.20990 \\
\hline $\mathrm{H}$ & 1.81017 & 2.48544 & -0.45191 \\
\hline $\mathrm{C}$ & 3.52401 & 3.41454 & -1.29569 \\
\hline $\mathrm{H}$ & 3.42521 & 2.95106 & -2.28957 \\
\hline $\mathrm{H}$ & 3.04245 & 4.40453 & -1.33266 \\
\hline $\mathrm{H}$ & 4.59838 & 3.57468 & -1.10896 \\
\hline $\mathrm{C}$ & 2.98256 & 3.21398 & 1.16419 \\
\hline $\mathrm{H}$ & 4.03332 & 3.36735 & 1.46118 \\
\hline $\mathrm{H}$ & 2.48499 & 4.19599 & 1.15682 \\
\hline $\mathrm{H}$ & 2.49726 & 2.60116 & 1.93811 \\
\hline
\end{tabular}


[( $\left.{ }^{\text {Dip }} \mathbf{L G a}\right)_{2}$ ], singlet, pbe0/def2-tzvp 168

Ga $\quad 0.22011 \quad 1.14836$

$-0.82779$

3.51417

0.54199

$-0.07367$

3.56026

-0.06397
2.35954

1.26524

2.77577

$-0.04076$

$-0.11885 \quad 2.33865$

3.05083

$-0.12966 \quad 1.39590$

3.05083

$-0.14811$

2.31388
1.35437

4.44100

$-0.18556$

3.50821

4.96282

$-0.13001$

3.48781

$-0.08020$

4.72486

5.16453

6.25697

6.25697
4.48939

$-0.05381 \quad 4.75248$

3.09424

$-0.02260$

5.71405

2.57936

0.11326

0.00509

$-1.52496 \quad 5.82366$

$-2.41155 \quad 5.19458$

0.10555

$-1.67481 \quad 7.18929$

$-0.23239$

$-2.67734 \quad 7.61465$

$-0.55236$

$-0.67270$

8.00758

$-0.32053$

$-0.35373$

$-0.53796$

0.72412

7.45911

$-0.23255$

1.60677
0.88029

8.09757

$-0.31861$

0.00295

0.10463

$-2.50425$

5.67486

$-0.46227$

$-4.88963$

2.47937

0.43616

0.00334

0.70400

3.08769

$-1.29410$

$-6.20891$

3.23990

$\begin{array}{ll}-4.12711 & 3.27886\end{array}$

$-2.18607$

$-3.21556$

$-2.79468$

3.57375
3.09990

-1.80245
1.85839

2.01214

$-2.27462$

$-4.13144$

-5.21889
-3.87361

1.76119

1.93272

2.19395

2.18141

$-3.91833$

0.92474

3.20451
1.48787

$-3.63061$

$-0.06984$

$-3.03666$

3.12513

2.86716

2.65915

3.89078

$\begin{array}{lll}-4.69511 & 3.41165 & 2.84701\end{array}$

$\begin{array}{lll}-1.71646 & 3.28578 & -2.85599\end{array}$

$\begin{array}{ll}-0.74330 & 3.21719\end{array}$

$-2.34543$

$-3.89509$

$-3.42019$

$-1.66728 \quad 1.17420$

$-4.62840$

.2 .27140
-95237

$-4.44660$

$-3.53286$

$-4.10768$

$\begin{array}{ll}-2.70289 & 4.78980\end{array}$

$-0.93482$

4.76945

$-4.23950$

$-1.70946$

$-2.79892$

$-0.12915$

0.87398

0.72741

1.51002

$-0.38546$

$-0.47792$

$-1.39244$

$-2.28331$

4.45892

4.82071

3.10399

3.15729

2.08906

3.74380

3.55529
4.98999

4.98999

3.34456

4.40361

3.01128

2.76601

2.18807

1.16358

2.21393

3.22885

1.53408
1.90658

2.49387

2.53217

1.71240

3.45217

4.11749

4.63557

3.79210

1.29590

2.09510

$\begin{array}{ll}1.70874 & 1.99241 \\ 0.60631 & 2.15965\end{array}$

$0.00552 \quad 1.25133$

$0.01197 \quad 3.01864$

$\begin{array}{ll}0.74894 & 2.27689\end{array}$

$2.73609 \quad 3.38735$

$2.99663 \quad 3.54757$

$2.14837 \quad 4.25697$

3.67195

4.25697
3.37414

$-2.46975$

$\begin{array}{ll}4.16524 & -2.07646 \\ 2.93938 & -3.47472\end{array}$

$-3.88007$

$-4.31944$

$-3.00843$

1.98973

$-2.46761$

$-3.91738$

$-3.71607$

(continued)

$\begin{array}{lr}\mathrm{Ga} & 0.22 \\ \mathrm{P} & -0.048 \\ \mathrm{~N} & 1.26 \\ \mathrm{C} & -0.0757\end{array}$

0.22053

1.26436
-0.07574

$-1.16516$

$-0.12448$

$-0.13755$

$-0.15411$

-0.19435

$-0.15767$

$-0.07930$

$-0.06188$

$-0.05245$

$-0.01832$

$-0.24878$

0.87383

1.87840

0.71494
1.59644

$-0.56273$

$-0.68520$

$-1.68364$

$-2.68708$

$-1.53106$

$-2.41649$

2.63091

3.55476

4.9017

5.60870

5.36009

6.41546

4.4612

4.82505
3.10614

3.15251

2.08416

3.89754

4.98400

3.54849

3.73719

3.33929

2.76221

3.00396

4.39853

2.19283

1.16766

2.21894

1.90920

1.54107

3.23445

2.50190

3.46070

1.72164

2.54125

$-2.50408$

$-3.57704$

$-4.89036$

$-5.71252$

$-5.17290$

$-6.20624$

$-4.12331$

$-4.34191$

$-2.79167$

$-3.34517$

-2.27935
-4.13773

$-3.92443$

$-3.88181$

$-5.22496$

$-3.63531$

$-4.69916$

$-3.39716$

$-3.03892$

$-1.71139$

$-0.73914$

$-1.76155$

$-2.71474$

$-0.94513$

$-1.66250$

$-1.76508$

$-1.70236$

$-0.92626$

$-2.69452$

$-1.14763$

$-3.51398$

$-2.77666$

$-3.56149$

$-2.52351$

$-2.34063$

$-1.39746$

$-2.31719$

. 3582

$-3.51212$

$-3.49278$

-4.72804
-5.66705

$-4.75433$

$-5.71534$

$-5.26284$

$-6.09433$

$-5.67631$

$-7.45865$

$-8.09788$

$-8.00552$

$-9.07554$

$-7.18625$

$-7.61038$

$-5.82128$

$-5.19151$

$-3.12473$

$-2.74479$

$-3.09771$

$-2.81319$

$-3.78861$

$-4.05739$

$-4.11707$

$-4.63488$

$-3.79170$

$-1.93923$

$-1.70860$

$-0.60432$

$-0.74580$

$-0.00960$

$-0.00444$ 
[( $\left.\left.{ }^{\text {Dip }} \mathbf{L G a}\right)_{2}\right]$, singlet, pbe0-gd3-bj/def2-tzvp 168

$\begin{array}{llrr}\mathrm{Ga} & 1.06630 & 0.20604 & 0.81920 \\ \mathrm{P} & 3.33918 & -0.04176 & -0.65730 \\ \mathrm{~N} & 2.35549 & -1.16410 & -0.05880 \\ \mathrm{C} & 3.31681 & -0.00837 & -2.46420 \\ \mathrm{~N} & 2.65231 & 1.24904 & 0.01286\end{array}$

$\begin{array}{lll}2.65231 & 1.24904 & 0.01286 \\ 2.07274 & -0.08171 & -3.10921\end{array}$

$\begin{array}{lll}1.15505 & -0.14120 & -2.51630\end{array}$

$\begin{array}{lll}1.99867 & -0.07057 & -4.49661\end{array}$

$\begin{array}{lll}1.02265 & -0.13023 & -4.98436 \\ 3.16515 & 0.01493 & -5.25930\end{array}$

$\begin{array}{lll}3.16515 & 0.01493 & -5.25930 \\ 3.10697 & 0.02025 & -6.35043\end{array}$

$\begin{array}{lll}4.40362 & 0.09407 & -4.62707\end{array}$

$\begin{array}{lll}5.31880 & 0.16221 & -5.21967\end{array}$

$\begin{array}{lll}4.48182 & 0.08142 & -3.23441\end{array}$

$\begin{array}{lll}5.45733 & 0.13467 & -2.74817\end{array}$

$\begin{array}{lll}5.08497 & -0.30089 & -0.28795 \\ 5.62033 & -1.59609 & -0.31017\end{array}$

$\begin{array}{lll}4.97709 & -2.45577 & -0.50605\end{array}$

$\begin{array}{lll}6.97905 & -1.79088 & -0.07249\end{array}$

$\begin{array}{lll}7.38845 & -2.80350 & -0.08032\end{array}$

$\begin{array}{lll}7.81058 & -0.69919 & 0.17632 \\ 8.87549 & -0.85551 & 0.36461\end{array}$

$\begin{array}{lll}7.28530 & 0.59269 & 0.17652\end{array}$

$7.93651-1.45058-0.35933$

$\begin{array}{llr}5.92704 & 0.79410 & -0.05903\end{array}$

$\begin{array}{rrr}5.51985 & 1.80764 & -0.06821 \\ 2.58973 & -2.49513 & 0.32767\end{array}$

$\begin{array}{lll}2.58973 & -2.49513 & -0.32767 \\ 2.28693 & -3.55884 & -0.55494\end{array}$

$\begin{array}{lll}2.54203 & -4.87156 & -0.14869\end{array}$

$\begin{array}{lll}2.31884 & -5.69264 & -0.83436 \\ 3.06181 & -5.15159 & 1.10979\end{array}$

$3.25509-6.18414$

$\begin{array}{lll}3.31216 & -4.10594 & 1.99082\end{array}$

$\begin{array}{lll}3.69406 & -4.32614 & 2.99122\end{array}$

$\begin{array}{lll}3.07956 & -2.77670 & 1.62892\end{array}$

$\begin{array}{lll}1.71425 & -3.29482 & -1.93031 \\ 1.39620 & -2.24286 & -1.93388\end{array}$

$\begin{array}{lll}1.39620 & -2.24286 & -1.93388\end{array}$

$\begin{array}{lll}0.48273 & -4.14676 & -2.22512 \\ 0.72369 & -5.22078 & -2.27132\end{array}$

$\begin{array}{lll}0.05113 & -3.86979 & -3.19905\end{array}$

$\begin{array}{lll}-0.29712 & -4.00956 & -1.46191\end{array}$

$\begin{array}{lll}2.78311 & -3.46427 & -3.00982\end{array}$

$\begin{array}{lll}3.65631 & -2.82243 & -2.81955\end{array}$

$\begin{array}{lll}2.38597 & -3.19523 & -4.00109 \\ 3.13478 & -4.50812 & -3.05419\end{array}$

$\begin{array}{lll}3.31178 & -1.68570 & 2.65618\end{array}$

$\begin{array}{lll}3.19424 & -0.72032 & 2.14043\end{array}$

$\begin{array}{lll}2.24744 & -1.74794 & 3.75340 \\ 1.23591 & -1.65920 & 3.32785\end{array}$

$\begin{array}{lll}2.38364 & -0.93056 & 4.47872\end{array}$

$\begin{array}{lll}2.29773 & -2.70233 & 4.30141\end{array}$

$\begin{array}{lll}4.72050 & -1.70239 & 3.24525\end{array}$

$\begin{array}{lll}4.91555 & -2.62598 & 3.81301\end{array}$

$\begin{array}{lll}4.85467 & -0.85725 & 3.93875\end{array}$

$\begin{array}{lll}5.48409 & -1.62067 & 2.45851\end{array}$

$\begin{array}{lll}5.94624 & 2.62031 & 0.11244\end{array}$

$\begin{array}{lll}2.44772 & 3.54702 & -0.83200 \\ 2.71629 & 4.90790 & -0.65141\end{array}$

$\begin{array}{lll}2.33788 & 5.62336 & -1.38599\end{array}$

$\begin{array}{lll}2.33788 & 5.62336 & -1.38599 \\ 3.43915 & 5.36711 & 0.44044\end{array}$

$\begin{array}{lll}3.63883 & 6.43435 & 0.56216\end{array}$

$\begin{array}{lll}3.89187 & 4.45580 & 1.38909\end{array}$

$\begin{array}{lll}4.43676 & 4.81938 & 2.26288\end{array}$

$\begin{array}{lll}3.65178 & 3.08727 & 1.25439\end{array}$

$\begin{array}{lll}1.61422 & 3.11837 & -2.02081\end{array}$

$\begin{array}{lll}1.41687 & 2.04426 & -1.90417 \\ 0.25751 & 3.82075 & -2.05454\end{array}$

0.25751

$\begin{array}{lll}-0.31991 & 3.64599 & -1.13463 \\ -0.34298 & 3.44833 & -2.89765\end{array}$

$\begin{array}{lll}0.35913 & 4.91040 & -2.17941\end{array}$

$\begin{array}{lll}2.37218 & 3.31862 & -3.33201\end{array}$

$\begin{array}{lll}2.59596 & 4.38445 & -3.50169\end{array}$

$\begin{array}{lll}1.77561 & 2.95933 & -4.18456\end{array}$

$\begin{array}{lll}3.32504 & 2.76916 & -3.33433\end{array}$

$\begin{array}{lll}4.07353 & 2.13929 & 2.36186 \\ 4.18724 & 1.14215 & 1.91470\end{array}$

4.18724

$\begin{array}{lll}2.97469 & 2.03449 & 3.42054 \\ 2.77306 & 3.01811 & 3.87364\end{array}$

$\begin{array}{lll}3.26904 & 1.34061 & 4.22381\end{array}$

$\begin{array}{lll}2.03077 & 1.66691 & 2.98633\end{array}$

$5.40706 \quad 2.50021-3.00931$

$\begin{array}{lll}6.20076 & 2.64093 & 2.26081\end{array}$

$\begin{array}{lll}5.72572 & 1.69636 & 3.69058 \\ 5.34060 & 3.42308 & 3.60671\end{array}$ (continued)

$\mathrm{Ga} \quad-1.06632$

$\mathrm{P} \quad-3.33915$

-3.33915
-2.65234
-3.31673

0.20597

$-0.04174$

1.24899

$-0.00806$

$-3.31673$

$-1.16413$

$-0.08100$

$-0.14039$

$-0.06953$

$-1.15493$

$-0.12888$

$-1.02247$

0.01589

0.02147

0.09466

0.16276

0.08171

0.13469

$-0.30094$

0.79400

1.80754

0.59253

1.45039

$-0.69935$

$-0.85571$

$-1.79099$

$-2.80361$

$-1.59614$

$-2.45578$

2.62025

3.54698

4.90787

5.62334

5.36705

6.43430

4.45572

4.81928

3.08718

3.11832

2.04420

3.82062

4.91027

3.44817

3.64580

3.31863

2.76922

2.95931

4.38448

. 13914

2.13914
1.14207

1.14207
2.03402

1.66632

1.34010

3.01755

2.50021

3.42300

1.69633

2.64115

$-2.49513$

$-3.55898$

$-4.87163$

$-5.69282$

$-5.15146$

$-6.18395$

$-4.10567$

$-4.32570$

$-2.77650$

$-3.29525$

$-2.24331$

$-4.14731$

$-4.01000$

$-3.87057$

$-5.22133$

$-3.46489$

$-4.50874$

$-3.19608$

$-2.82298$

$-1.68533$

$-0.72004$

$-1.74741$

$-2.70169$

$-0.92989$

$-1.65879$

$-1.70187$

$-1.62026$

$-0.85661$

$-2.62536$
$-0.01293$

2.46430

0.05896

3.10930

2.51637

4.49669

.98442

4.62718

5.21980

23453

2.74830

0.28815

0.05904

.06804

$-0.17646$

0.35940

$-0.17601$

-0.36427
0.07298

0.08099

0.31060

0.50664

$-0.11259$

0.83180

0.65115

1.38569

$-0.44074$

$-0.56251$

$-1.38933$

$-2.26314$

$-1.25455$

2.0206

1.90401

.05430

2.17915

2.89740

.13438

3.33184

3.33418

4.18437

3.5015

$-2.36194$

$-1.91467$

$-3.42055$

$-2.98626$

$-4.22375$

$-3.87377$

$-3.00949$

$-3.60700$

$-3.69068$

$-2.26105$

$-0.32759$

0.55488

0.14844

0.83399

$-1.11007$

$-1.41040$

$-1.99095$

$-2.99138$

$-1.62886$

1.93027

1.93404

2.22488

1.46168

3.19886

2.27087

3.00978

3.05393

4.00109

2.81967

$-2.65597$

$-2.14005$

$-4.47830$

$-3.32748$

$-3.24514$

$-2.45846$

-3.93851
-3.81307 
[ ${ }^{\text {Dip }}$ LIn:], singlet, pbe0/def2-tzvp 84

\section{$\begin{array}{llll}\text { In } & 0.14498 & -0.36104 & -250054\end{array}$} 0.14498

$\begin{array}{lll}1.24419 & -0.12962 & -0.46448\end{array}$

$\begin{array}{lll}0.08125 & 2.10053 & 0.87362\end{array}$

$\begin{array}{lll}-1.22807 & 0.11350 & -0.66094\end{array}$

$\begin{array}{lll}0.00654 & 3.07850 & -0.13010\end{array}$

$\begin{array}{lll}-0.12411 & 2.77649 & -1.17319\end{array}$

$\begin{array}{lll}0.08670 & 4.43102 & 0.18278\end{array}$

$\begin{array}{lll}0.02119 & 5.17789 & -0.61228\end{array}$

$\begin{array}{lll}0.24768 & 4.83240 & 1.51082\end{array}$

$\begin{array}{lll}0.30806 & 5.89494 & 1.75878\end{array}$

$\begin{array}{lll}0.33083 & 3.87289 & 2.51577 \\ 0.45817 & 4.17853 & 3.55713\end{array}$

$\begin{array}{lll}0.24760 & 2.51525 & 2.20034\end{array}$

$\begin{array}{lll}0.30774 & 1.77554 & 3.00061\end{array}$

$\begin{array}{lll}-0.27474 & -0.46831 & 1.99533\end{array}$

$\begin{array}{lll}0.82051 & -0.95578 & 2.72128\end{array}$

2.29881

$\begin{array}{lll}0.63644 & -1.50391 & 3.99016 \\ 1.49830 & -1.88094 & 4.54627\end{array}$

$\begin{array}{lll}-0.63964 & -1.56564 & 4.54827\end{array}$

$\begin{array}{lll}-0.78235 & -1.99635 & 5.54254\end{array}$

$\begin{array}{lll}-1.73230 & -1.06819 & 3.83857\end{array}$

$\begin{array}{lll}-2.73391 & -1.10678 & 4.27338\end{array}$

$\begin{array}{lll}-1.55302 & -0.51666 & 2.57119\end{array}$

$\begin{array}{lll}-2.41632 & -0.12456 & 2.03070\end{array}$

$\begin{array}{lll}2.60565 & -0.33535 & -0.16826 \\ 3.56582 & 0.69719 & -0.31928\end{array}$

$\begin{array}{lll}3.56582 & 0.69719 & -0.31928\end{array}$

$\begin{array}{lll}4.90894 & 0.43137 & -0.02899\end{array}$

$\begin{array}{lrr}5.64213 & 1.23588 & -0.13474 \\ 5.33221 & -0.82575 & 0.37703\end{array}$

$\begin{array}{lll}6.38450 & -1.01036 & 0.60638\end{array}$

$\begin{array}{lll}4.40159 & -1.85627 & 0.46012\end{array}$

$\begin{array}{lll}4.73835 & -2.85696 & 0.73974 \\ 3.04976 & -1.64459 & 0.18087\end{array}$

$3.20363 \quad 2.06744 \quad-0.85757$

$\begin{array}{lll}2.11342 & 2.08390 & -0.97531\end{array}$

$\begin{array}{lll}3.81372 & 2.28744 & -2.24450\end{array}$

$\begin{array}{lll}4.91504 & 2.28700 & -2.20704 \\ 3.49550 & 3.25684 & -2.66138 \\ 3.50788 & 1.49728 & -2.94802\end{array}$

$\begin{array}{lll}3.50788 & 1.49728 & -2.94802\end{array}$

$\begin{array}{lll}3.57883 & 3.19773 & 0.09930\end{array}$

$\begin{array}{lll}3.10034 & 3.06683 & 1.08138\end{array}$

$\begin{array}{lll}3.25623 & 4.17037 & -0.30425\end{array}$

$\begin{array}{lll}4.66800 & 3.25145 & 0.25844\end{array}$

$\begin{array}{lll}2.09855 & -2.82894 & 0.16448\end{array}$

$\begin{array}{lll}1.08772 & -2.44115 & 0.35692\end{array}$

$\begin{array}{lll}2.08523 & -3.46874 & -1.22754 \\ 1.82224 & -2.73963 & -2.01094\end{array}$

$\begin{array}{lll}1.82224 & -2.73963 & -2.01094 \\ 3.35917 & -4.29641 & -1.27811\end{array}$

$\begin{array}{lll}1.35917 & -4.29641 & -1.27811 \\ 3.07879 & -3.87138 & -1.48219\end{array}$

$\begin{array}{lll}2.38983 & -3.88606 & 1.22751\end{array}$

$\begin{array}{lll}3.33422 & -4.41985 & 1.03598\end{array}$

$\begin{array}{lll}1.59029 & -4.64306 & 1.23583\end{array}$

$\begin{array}{lll}2.44794 & -3.44948 & 2.23573\end{array}$

$\begin{array}{lll}-2.57222 & -0.26583 & -0.48380\end{array}$

$\begin{array}{lll}-3.60530 & 0.70755 & -0.45811 \\ -4.93218 & 0.29757 & -0.29795\end{array}$

$-4.93218$

$\begin{array}{lrr}-5.72079 & 1.05469 & -0.26686 \\ -5.27053 & -1.04511 & -0.18454\end{array}$

$\begin{array}{lll}-6.31332 & -1.34638 & -0.05874\end{array}$

$\begin{array}{lll}-4.26350 & -2.00051 & -0.24876\end{array}$

$\begin{array}{lll}-4.52661 & -3.05969 & -0.18030\end{array}$

$\begin{array}{lll}-2.92075 & -1.64292 & -0.40043\end{array}$

$\begin{array}{lll}-3.30797 & 2.18663 & -0.59769\end{array}$

$\begin{array}{lll}-2.23916 & 2.26469 & -0.83253 \\ -4.07858 & 2.82481 & -1.75411\end{array}$

$\begin{array}{lll}-4.07858 & 2.82481 & -1.75411 \\ -3.89539 & 2.29279 & -2.70025\end{array}$

$\begin{array}{lll}-3.89539 & 2.29279 & -2.70025 \\ -3.77339 & 3.87507 & -1.88853 \\ -5.16616 & 2.82193 & -1.57669\end{array}$

$\begin{array}{lll}-5.16616 & 2.82193 & -1.57669\end{array}$

$\begin{array}{lll}-3.54708 & 2.93994 & 0.71168\end{array}$

$\begin{array}{lll}-4.60731 & 2.89760 & 1.01113\end{array}$

$\begin{array}{lll}-3.26769 & 4.00091 & 0.61042\end{array}$

$\begin{array}{lll}-2.94907 & 2.51771 & 1.53375\end{array}$

$\begin{array}{lll}-1.88719 & -2.74980 & -0.51273 \\ -0.89584 & -2.27066 & -0.49127\end{array}$

$\begin{array}{lll}-2.01918 & -3.48519 & -1.84989\end{array}$

$\begin{array}{lll}-2.99383 & -3.99322 & -1.92871\end{array}$

$\begin{array}{lll}-1.23252 & -4.24899 & -1.96006\end{array}$

$\begin{array}{lll}-1.94369 & -2.79392 & -2.70460\end{array}$

$\begin{array}{lll}-1.93061 & -3.73452 & 0.65617\end{array}$

$\begin{array}{lll}-1.80608 & -3.22064 & 1.62051\end{array}$

$\begin{array}{lll}-1.12424 & -4.47938 & 0.56117 \\ -2.88235 & -4.28861 & 0.68874\end{array}$
[ ${ }^{\text {Dip }}$ LIn:], singlet, pbe0-gd3-bj/def2-tzvp 84

$\begin{array}{lccc}\text { In } & 0.17047 & -0.32404 & -2.55092 \\ \mathrm{P} & -0.03834 & 0.35192 & 0.34559 \\ \mathrm{~N} & 1.24605 & -0.13368 & -0.48516 \\ \mathrm{C} & 0.08198 & 2.09375 & 0.84881\end{array}$

$\begin{array}{llll}\mathrm{C} & 0.08198 & 2.09375 & 0.84881 \\ \mathrm{~N} & -1.21407 & 0.15786 & -0.72839\end{array}$

$\begin{array}{lll}-1.21407 & 0.15786 & -0.72839 \\ -0.00508 & 3.07997 & -0.14395\end{array}$

$\begin{array}{lll}-0.14381 & 2.78490 & -1.18759\end{array}$

$\begin{array}{lll}0.07113 & 4.42762 & 0.18565\end{array}$

$0.23647-4.81158$

$\begin{array}{lll}0.29210 & 5.87095 & 1.77959\end{array}$

$\begin{array}{lll}0.33047 & 3.84090 & 2.51162\end{array}$

$\begin{array}{lll}0.46147 & 4.13524 & 3.55549\end{array}$

$\begin{array}{lll}0.25430 & 2.48747 & 2.17972\end{array}$

$\begin{array}{lll}0.32061 & 1.73452 & 2.96699\end{array}$

$\begin{array}{lll}-0.33105 & -0.47963 & 1.92538\end{array}$

$\begin{array}{lll}0.73619 & -1.03356 & 2.64344 \\ 1.74911 & -0.98807 & 2.23953\end{array}$

$\begin{array}{lll}1.74911 & -0.98807 & 2.23953\end{array}$

$\begin{array}{lll}0.51224 & -1.63665 & 3.87963\end{array}$

$\begin{array}{lll}1.35120 & -2.06922 & 4.42980\end{array}$

$\begin{array}{lll}-0.77520 & -1.68382 & 4.41230 \\ -0.95008 & -2.16129 & 5.37946\end{array}$

$\begin{array}{lll}-1.83779 & -1.11072 & 3.71413\end{array}$

$\begin{array}{lll}-2.84725 & -1.13582 & 4.13058\end{array}$

$\begin{array}{lll}-1.61942 & -0.50459 & 2.47936\end{array}$

$\begin{array}{lll}-2.45918 & -0.05747 & 1.94615\end{array}$

$\begin{array}{lll}2.59261 & -0.33404 & -0.13907\end{array}$

$\begin{array}{lll}3.54602 & 0.70681 & -0.23246\end{array}$

$\begin{array}{lll}4.87655 & 0.44655 & 0.11257\end{array}$

$\begin{array}{rrr}5.60797 & 1.25695 & 0.05026 \\ 5.28770 & -0.81444 & 0.52030\end{array}$

$\begin{array}{lll}6.32991 & -0.99617 & 0.79289\end{array}$

$\begin{array}{lll}4.36093 & -1.85231 & 0.55410\end{array}$

$\begin{array}{lll}4.68981 & -2.85311 & 0.84154 \\ 3.02263 & -1.64271 & 0.21887\end{array}$

$\begin{array}{lll}3.02263 & -1.64271 & 0.21887 \\ 3.18262 & 2.07654 & -0.76345\end{array}$

$\begin{array}{lll}3.18262 & 2.07654 & -0.76345 \\ 2.09968 & 2.07133 & -0.93365\end{array}$

$\begin{array}{lll}2.09968 & 2.07133 & -0.93365\end{array}$

$\begin{array}{lll}3.85085 & 2.32949 & -2.11546\end{array}$

$\begin{array}{lll}4.94894 & 2.34991 & -2.02697\end{array}$

$\begin{array}{lll}3.53137 & 3.29781 & -2.53329\end{array}$

$\begin{array}{lll}3.59243 & 1.54185 & -2.84030\end{array}$

$\begin{array}{lll}3.48596 & 3.19288 & 0.23147\end{array}$

$\begin{array}{lll}2.96089 & 3.02944 & 1.18396\end{array}$

$\begin{array}{lll}3.16248 & 4.16688 & -0.16706\end{array}$

$\begin{array}{lll}4.56449 & 3.26273 & 0.44615\end{array}$

$\begin{array}{lll}2.06045 & -2.81404 & 0.16349\end{array}$

$\begin{array}{lll}1.05530 & -2.41827 & 0.36493\end{array}$

$\begin{array}{lll}2.03993 & -3.40755 & -1.24617\end{array}$

$\begin{array}{lll}1.78679 & -2.64763 & -2.00268\end{array}$

$\begin{array}{lll}1.30185 & -4.22171 & -1.32414\end{array}$

$\begin{array}{lll}3.02837 & -3.81340 & -1.51359\end{array}$

$\begin{array}{lll}2.33674 & -3.90125 & 1.19623\end{array}$

$\begin{array}{lll}3.27569 & -4.43928 & 0.99158\end{array}$

$\begin{array}{lll}1.52820 & -4.64803 & 1.18453\end{array}$

$\begin{array}{lll}2.39796 & -3.49001 & 2.21472\end{array}$

$\begin{array}{lll}-2.54010 & -0.25743 & -0.52143\end{array}$

$\begin{array}{lll}-3.58834 & 0.69298 & -0.45697 \\ -4.89647 & 0.25395 & -0.23709\end{array}$

$-5.70143$

$\begin{array}{lll}-5.19338 & -1.09711 & -0.09874\end{array}$

$\begin{array}{lll}-6.22166 & -1.42239 & 0.07573\end{array}$

$\begin{array}{lll}-4.16792 & -2.03048 & -0.19753\end{array}$

$\begin{array}{lll}-4.40060 & -3.09483 & -0.10580\end{array}$

$\begin{array}{lll}-2.84369 & -1.63976 & -0.40989\end{array}$

$\begin{array}{lll}-3.30536 & 2.17395 & -0.58726\end{array}$

$\begin{array}{lll}-2.26685 & 2.25588 & -0.93360\end{array}$

$2.34650-2.60295$

$\begin{array}{lll}-3.89382 & 3.90266 & -1.76359 \\ -5.25411 & 2.85232 & -1.32777\end{array}$

$\begin{array}{lll}-3.39615 & 2.87804 & 0.76630\end{array}$

$\begin{array}{lll}-4.41933 & 2.82402 & 1.17283\end{array}$

$\begin{array}{lll}-3.12008 & 3.94058 & 0.67662\end{array}$

$\begin{array}{lll}-2.71658 & 2.42345 & 1.50229\end{array}$

$\begin{array}{lll}-1.77628 & -2.70726 & -0.55029\end{array}$

$\begin{array}{lll}-0.80366 & -2.19199 & -0.56603\end{array}$

$\begin{array}{lll}-1.92794 & -3.44874 & -1.87980\end{array}$

$\begin{array}{lll}-2.88678 & -3.98940 & -1.92409\end{array}$

$-1.11834 \quad-4.18281 \quad-2.01800$

$\begin{array}{lll}-1.90714 & -2.75348 & -2.73425\end{array}$

$\begin{array}{lll}-1.73896 & -3.68152 & 0.62498\end{array}$

$\begin{array}{lll}-1.58987 & -3.15349 & 1.57762\end{array}$

$\begin{array}{lll}-0.91344 & -4.39976 & 0.50046\end{array}$

$\begin{array}{lll}-2.67052 & -4.26424 & 0.70211\end{array}$ 


\begin{tabular}{|c|c|c|c|}
\hline \multicolumn{4}{|c|}{ [ ${ }^{\text {Dip }}$ LIn:], triplet, pbe0/def2-tzvp 84} \\
\hline In & 0.14235 & -0.02868 & -2.45649 \\
\hline $\mathrm{P}$ & -0.03291 & 0.31248 & 0.39125 \\
\hline $\mathrm{N}$ & 1.25314 & -0.13699 & -0.48073 \\
\hline $\mathrm{C}$ & 0.06961 & 2.03979 & 0.95895 \\
\hline $\mathrm{N}$ & -1.23490 & 0.12408 & -0.67413 \\
\hline $\mathrm{C}$ & -0.03652 & 3.07609 & 0.01665 \\
\hline $\mathrm{H}$ & -0.17374 & 2.83137 & -1.04171 \\
\hline $\mathrm{C}$ & 0.04239 & 4.40607 & 0.41629 \\
\hline $\mathrm{H}$ & -0.04333 & 5.20043 & -0.32927 \\
\hline $\mathrm{C}$ & 0.22660 & 4.72473 & 1.76324 \\
\hline $\mathrm{H}$ & 0.28265 & 5.77018 & 2.07671 \\
\hline $\mathrm{C}$ & 0.33913 & 3.70558 & 2.70524 \\
\hline $\mathrm{H}$ & 0.48525 & 3.94653 & 3.76094 \\
\hline $\mathrm{C}$ & 0.26259 & 2.37014 & 2.30656 \\
\hline $\mathrm{H}$ & 0.34838 & 1.58301 & 3.05757 \\
\hline $\mathrm{C}$ & -0.28935 & -0.59055 & 1.93881 \\
\hline $\mathrm{C}$ & 0.78185 & -1.19078 & 2.61259 \\
\hline $\mathrm{H}$ & 1.78435 & -1.16527 & 2.18194 \\
\hline $\mathrm{C}$ & 0.57664 & -1.81025 & 3.84477 \\
\hline $\mathrm{H}$ & 1.41939 & -2.27522 & 4.36204 \\
\hline $\mathrm{C}$ & -0.69470 & -1.83096 & 4.41646 \\
\hline $\mathrm{H}$ & -0.85325 & -2.31868 & 5.38148 \\
\hline $\mathrm{C}$ & -1.76194 & -1.21960 & 3.75850 \\
\hline $\mathrm{H}$ & -2.75921 & -1.22555 & 4.20447 \\
\hline $\mathrm{C}$ & -1.56262 & -0.59581 & 2.52912 \\
\hline $\mathrm{H}$ & -2.40585 & -0.11405 & 2.03090 \\
\hline $\mathrm{C}$ & 2.61403 & -0.35323 & -0.19659 \\
\hline $\mathrm{C}$ & 3.56228 & 0.70026 & -0.26587 \\
\hline $\mathrm{C}$ & 4.90108 & 0.43014 & 0.03622 \\
\hline $\mathrm{H}$ & 5.62796 & 1.24584 & -0.00258 \\
\hline $\mathrm{C}$ & 5.32764 & -0.84731 & 0.37060 \\
\hline $\mathrm{H}$ & 6.37696 & -1.03518 & 0.61119 \\
\hline $\mathrm{C}$ & 4.41040 & -1.89324 & 0.36596 \\
\hline $\mathrm{H}$ & 4.75525 & -2.90562 & 0.58669 \\
\hline $\mathrm{C}$ & 3.06233 & -1.68108 & 0.07069 \\
\hline $\mathrm{C}$ & 3.19642 & 2.09440 & -0.73456 \\
\hline $\mathrm{H}$ & 2.11029 & 2.11048 & -0.88812 \\
\hline $\mathrm{C}$ & 3.83901 & 2.39149 & -2.09203 \\
\hline $\mathrm{H}$ & 4.93935 & 2.39040 & -2.03091 \\
\hline $\mathrm{H}$ & 3.52334 & 3.38054 & -2.46038 \\
\hline $\mathrm{H}$ & 3.54409 & 1.64481 & -2.84572 \\
\hline $\mathrm{C}$ & 3.54036 & 3.17242 & 0.29168 \\
\hline $\mathrm{H}$ & 3.04555 & 2.98231 & 1.25578 \\
\hline $\mathrm{H}$ & 3.21236 & 4.16135 & -0.06434 \\
\hline $\mathrm{H}$ & 4.62567 & 3.23101 & 0.47423 \\
\hline $\mathrm{C}$ & 2.13146 & -2.87590 & -0.04407 \\
\hline $\mathrm{H}$ & 1.10808 & -2.51345 & 0.12874 \\
\hline $\mathrm{C}$ & 2.17849 & -3.43651 & -1.46908 \\
\hline $\mathrm{H}$ & 1.88404 & -2.67769 & -2.21152 \\
\hline $\mathrm{H}$ & 1.48693 & -4.28764 & -1.57725 \\
\hline $\mathrm{H}$ & 3.19327 & -3.78633 & -1.71914 \\
\hline $\mathrm{C}$ & 2.40856 & -3.98263 & 0.97142 \\
\hline $\mathrm{H}$ & 3.36411 & -4.49510 & 0.77695 \\
\hline $\mathrm{H}$ & 1.61946 & -4.74832 & 0.91728 \\
\hline $\mathrm{H}$ & 2.43482 & -3.60209 & 2.00360 \\
\hline $\mathrm{C}$ & -2.56775 & -0.29525 & -0.51185 \\
\hline $\mathrm{C}$ & -3.61103 & 0.66695 & -0.45467 \\
\hline $\mathrm{C}$ & -4.92750 & 0.23408 & -0.27418 \\
\hline $\mathrm{H}$ & -5.72815 & 0.97627 & -0.21460 \\
\hline $\mathrm{C}$ & -5.23873 & -1.11672 & -0.17609 \\
\hline $\mathrm{H}$ & -6.27380 & -1.43641 & -0.03201 \\
\hline $\mathrm{C}$ & -4.22133 & -2.05818 & -0.28208 \\
\hline $\mathrm{H}$ & -4.46889 & -3.12186 & -0.23075 \\
\hline $\mathrm{C}$ & -2.88761 & -1.68034 & -0.45686 \\
\hline $\mathrm{C}$ & -3.33602 & 2.15133 & -0.58224 \\
\hline $\mathrm{H}$ & -2.27299 & 2.24952 & -0.83680 \\
\hline $\mathrm{C}$ & -4.13748 & 2.78821 & -1.71844 \\
\hline $\mathrm{H}$ & -3.95933 & 2.27104 & -2.67349 \\
\hline $\mathrm{H}$ & -3.84953 & 3.84386 & -1.84606 \\
\hline $\mathrm{H}$ & -5.22170 & 2.76629 & -1.52266 \\
\hline $\mathrm{C}$ & -3.56752 & 2.88566 & 0.73946 \\
\hline $\mathrm{H}$ & -4.62182 & 2.82124 & 1.05540 \\
\hline $\mathrm{H}$ & -3.30938 & 3.95224 & 0.64380 \\
\hline $\mathrm{H}$ & -2.94783 & 2.46798 & 1.54774 \\
\hline $\mathrm{C}$ & -1.84252 & -2.76748 & -0.62535 \\
\hline $\mathrm{H}$ & -0.86165 & -2.27137 & -0.68908 \\
\hline $\mathrm{C}$ & -2.05080 & -3.51809 & -1.94364 \\
\hline $\mathrm{H}$ & -3.01735 & -4.04774 & -1.95660 \\
\hline $\mathrm{H}$ & -1.25467 & -4.26454 & -2.09363 \\
\hline $\mathrm{H}$ & -2.02715 & -2.83069 & -2.80303 \\
\hline $\mathrm{C}$ & -1.79989 & -3.74153 & 0.55289 \\
\hline $\mathrm{H}$ & -1.61619 & -3.22330 & 1.50507 \\
\hline $\mathrm{H}$ & -0.99656 & -4.48125 & 0.40768 \\
\hline $\mathrm{H}$ & -2.74345 & -4.30219 & 0.65154 \\
\hline
\end{tabular}


[( ${ }^{\text {Dip }}$ LIn $)_{2}$ ], singlet, pbe0/def2-tzvp 168

$\begin{array}{llll}\text { In } & 0.12024 & -1.31920 & -0.98863\end{array}$

$\begin{array}{ll}-3.83462 & 0.57286\end{array}$

$\begin{array}{lll}1.29173 & -3.10172 & -0.09517 \\ 0.08665 & -3.81034 & 2.39588\end{array}$

$\begin{array}{lll}-1.18664 & -2.93598 & 0.01098\end{array}$

$\begin{array}{lll}0.00216 & -2.57188 & 3.05147\end{array}$

$\begin{array}{lll}-0.09886 & -1.65112 & 2.46971\end{array}$

$\begin{array}{lll}0.04325 & -2.49696 & 4.43956\end{array}$

$\begin{array}{lll}-0.02707 & -1.52331 & 4.93105\end{array}$

$\begin{array}{lll}0.17130 & -3.66100 & 5.20042\end{array}$

$0.20032 \quad-3.60301 \quad 6.29134$

$\begin{array}{lll}0.26293 & -4.89491 & 4.56249\end{array}$

$\begin{array}{lll}0.36589 & -5.81060 & 5.14988\end{array}$

$0.22114--4.97036$

2.68536

$\begin{array}{rrr}-0.14867 & -5.61030 & 0.23752 \\ 0.97354 & -6.40237 & -0.03759\end{array}$

$1.96192 \quad-5.94413 \quad-0.11053$

$\begin{array}{lll}0.83799 & -7.78008 & -0.20683\end{array}$

$\begin{array}{lll}1.72058 & -8.38795 & -0.42084\end{array}$

$\begin{array}{lll}-0.41574 & -8.37952 & -0.09681\end{array}$

$\begin{array}{lll}-0.41574 & -9.379933 & -0.22938 \\ -1.53555 & -7.59946 & 0.19180\end{array}$

$\begin{array}{lll}-1.40495 & -6.22270 & 0.36357\end{array}$

$\begin{array}{lll}-2.28815 & -5.62510 & 0.59776\end{array}$

$\begin{array}{lll}2.66290 & -3.40879 & -0.21017\end{array}$

3.60539

$\begin{array}{lll}4.95710 & -3.30796 & 0.58881\end{array}$

$\begin{array}{lll}5.67581 & -2.99344 & 1.35063\end{array}$

$\begin{array}{lll}5.40695 & -3.99920 & -0.52663\end{array}$

$\begin{array}{lll}6.46614 & -4.24307 & -0.63946\end{array}$

$\begin{array}{lll}4.49287 & -4.35294 & -1.51318\end{array}$

$\begin{array}{lll}4.84822 & -4.86237 & -2.41194 \\ 3.13286 & -4.06076 & -1.38826\end{array}$

$\begin{array}{lll}3.21667 & -2.15367 & 1.96681\end{array}$

$\begin{array}{lll}2.13076 & -2.00673 & 1.91624\end{array}$

$\begin{array}{lll}3.86454 & -0.76786 & 1.91189\end{array}$

$\begin{array}{lll}4.96336 & -0.82743 & 1.96711\end{array}$

$\begin{array}{lll}3.52592 & -0.14608 & 2.75610\end{array}$

$-0.23687$

$\begin{array}{lll}3.52828 & -2.85571 & 3.28804\end{array}$

$\begin{array}{lll}3.01968 & -3.82893 & 3.35626\end{array}$

$\begin{array}{lll}3.19578 & -2.24309 & 4.14059\end{array}$

$\begin{array}{lll}2.20362 & -4.36605 & -2.55065\end{array}$

$\begin{array}{lll}1.18337 & -4.42617 & -2.14449\end{array}$

$\begin{array}{lll}2.23133 & -3.21310 & -3.55981 \\ 1.97451 & -2.25019 & -3.08933 \\ 1.51919 & -3.38806 & -4.38275\end{array}$

$\begin{array}{lll}1.51919 & -3.38806 & -4.38275\end{array}$

$\begin{array}{lll}3.23681 & -3.10210 & -3.99701\end{array}$

$\begin{array}{lll}2.49366 & -5.68964 & -3.25610\end{array}$

$\begin{array}{lll}3.45338 & -5.67021 & -3.79642\end{array}$

$-4.00145$

$\begin{array}{rrr}2.52066 & -6.53363 & -2.55126 \\ -2.53318 & -3.23464 & -0.27660\end{array}$

$\begin{array}{lll}-3.56107 & -2.92689 & 0.65278\end{array}$

$\begin{array}{rrr}-4.88994 & -3.21350 & 0.32520 \\ -5.67539 & -2.98452 & 1.05075\end{array}$

$\begin{array}{lll}-5.23428 & -3.77244 & -0.89883\end{array}$

$\begin{array}{lll}-6.27871 & -3.98978 & -1.13560\end{array}$

$\begin{array}{lll}-4.23213 & -4.03527 & -1.82503\end{array}$

$\begin{array}{ll}-4.45290 & -2.79924 \\ -3.77431 & -1.54509\end{array}$

$\begin{array}{ll}-3.77431 & -1.54509\end{array}$

$\begin{array}{lll}-3.26030 & -2.29521 & 1.99732 \\ -2.19406 & -2.03554 & 1.98560\end{array}$

$\begin{array}{lll}-4.04232 & -1.00006 & 2.22141\end{array}$

$\begin{array}{lll}-3.87321 & -0.27688 & 1.40895\end{array}$

$\begin{array}{lll}-3.73384 & -0.52358 & 3.16578\end{array}$

$\begin{array}{lll}-5.12783 & -1.17763 & 2.28600\end{array}$

$\begin{array}{lll}-3.48653 & -3.28078 & 3.14494\end{array}$

$-4.54432-3.58554$

$\begin{array}{lll}-3.21012 & -2.82783 & 4.11028\end{array}$

$\begin{array}{lll}-2.88079 & -4.19135 & 3.02102\end{array}$

$\begin{array}{lll}-1.86077 & -4.02857 & -2.63453 \\ -0.86662 & -3.92506 & -2.17314\end{array}$

$\begin{array}{lll}-1.97128 & -2.97348 & -3.73961\end{array}$

$\begin{array}{lll}-2.95121 & -3.02758 & -4.24085\end{array}$

$\begin{array}{lll}-1.19210 & -3.12034 & -4.50510\end{array}$

$\begin{array}{lll}-1.86547 & -1.95242 & -3.33917\end{array}$

$\begin{array}{lll}-1.93654 & -5.43728 & -3.22372\end{array}$

$\begin{array}{lll}-1.84193 & -6.20612 & -2.44329\end{array}$

$\begin{array}{lll}-1.12493 & -5.59156 & -3.95267\end{array}$

$\begin{array}{lll}-2.88629 & -5.60871 & -3.75516\end{array}$

(continued)

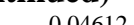

In $\quad-0.04612$

$\begin{array}{ll}1.31081 & 0.97949\end{array}$

$\begin{array}{lll}-0.03544 & 3.83243 & -0.57414\end{array}$

$\begin{array}{lll}-1.27733 & 3.06113 & 0.09910\end{array}$

$\begin{array}{lll}-0.10784 & 3.81225 & -2.39685\end{array}$

\begin{tabular}{lll}
-0.00803 & 2.96662 & -0.02283 \\
\hline & 2.57677 & -3.05597
\end{tabular}

$\begin{array}{lll}0.11095 & 1.65625 & -2.47721\end{array}$

$\begin{array}{lll}-0.05692 & 2.50438 & -4.44390\end{array}$

$\begin{array}{lll}0.02533 & 1.53305 & -4.93812\end{array}$

$\begin{array}{lll}-0.20800 & 3.66805 & -5.20117\end{array}$

$\begin{array}{lll}-0.24350 & 3.61207 & -6.29200\end{array}$

$\begin{array}{lll}-0.31450 & 4.89891 & -4.55974\end{array}$

$\begin{array}{lll}-0.43573 & 5.81419 & -5.14424\end{array}$

$-0.26530$

0.08841

$\begin{array}{rrr}0.08841 & 5.61068 & -0.23236 \\ -1.05143 & 6.36676 & 0.06966\end{array}$

$\begin{array}{lll}-2.02392 & 5.87847 & 0.15854\end{array}$

$\begin{array}{lll}-0.95390 & 7.74690 & 0.24562\end{array}$

$\begin{array}{lll}-1.85000 & 8.32659 & 0.48066\end{array}$

$\begin{array}{lll}0.27889 & 8.38442 & 0.11557\end{array}$

$\begin{array}{lll}0.35342 & 9.46605 & 0.25340\end{array}$

$\begin{array}{lll}0.31582 & 7.64024 & -0.19986\end{array}$

$\begin{array}{lll}2.38361 & 8.13528 & -0.31044\end{array}$

$\begin{array}{lll}1.32324 & 6.26132 & -0.37834\end{array}$

$\begin{array}{lll}2.21913 & 5.69206 & -0.63406\end{array}$

$\begin{array}{lll}-2.65370 & 3.33278 & 0.23943\end{array}$

$\begin{array}{lll}-3.60052 & 2.90987 & -0.72824\end{array}$

$\begin{array}{lll}-4.95687 & 3.19089 & -0.52760\end{array}$

$\begin{array}{lll}-5.67889 & 2.87394 & -1.28527\end{array}$

$\begin{array}{lll}-5.40685 & 3.84817 & 0.60815\end{array}$

$\begin{array}{lll}-6.46998 & 4.06267 & 0.74169\end{array}$

$\begin{array}{lll}-4.48666 & 4.20611 & 1.58736\end{array}$

$\begin{array}{lll}-4.84047 & 4.68933 & 2.50113\end{array}$

$\begin{array}{lll}-3.12179 & 3.95124 & 1.43626\end{array}$

$\begin{array}{lll}-3.20948 & 2.11319 & -1.95731\end{array}$

$\begin{array}{lll}-2.12095 & 1.98303 & -1.91974\end{array}$

$\begin{array}{lll}-3.83405 & 0.71590 & -1.93796\end{array}$

$\begin{array}{lll}-4.93418 & 0.75806 & -1.98133\end{array}$

$\begin{array}{lll}-3.49391 & 0.12635 & -2.80435\end{array}$

$\begin{array}{lll}-3.56287 & 0.16000 & -1.02716\end{array}$

$\begin{array}{lll}-3.54402 & 2.84793 & -3.25511\end{array}$

$\begin{array}{lll}-3.05401 & 3.83203 & -3.29841\end{array}$

$\begin{array}{lll}-3.20716 & 2.26693 & -4.12786\end{array}$

$\begin{array}{lll}-4.62971 & 3.00627 & -3.36059\end{array}$

$\begin{array}{lll}-2.18350 & 4.26191 & 2.58983\end{array}$

$\begin{array}{lll}-1.16974 & 4.34331 & 2.17124\end{array}$

$\begin{array}{lll}-2.17892 & 3.10032 & 3.58938\end{array}$

$\begin{array}{lll}-1.91312 & 2.14562 & 3.10734\end{array}$

$\begin{array}{lll}-1.45869 & 3.28001 & 4.40422\end{array}$

$\begin{array}{lll}-3.17654 & 2.96932 & 4.03895\end{array}$

$\begin{array}{lll}-2.48737 & 5.57442 & 3.31024\end{array}$

$\begin{array}{lll}-3.44095 & 5.53454 & 3.86022\end{array}$

$\begin{array}{lll}-1.70044 & 5.79179 & 4.04939\end{array}$

$\begin{array}{lll}-2.53538 & 6.42404 & 2.61341\end{array}$

$\begin{array}{lll}2.54662 & 3.30483 & 0.23967\end{array}$

$\begin{array}{lll}3.56747 & 3.01797 & -0.70371\end{array}$

$\begin{array}{lll}4.89225 & 3.34946 & -0.40248\end{array}$

$\begin{array}{lll}5.67192 & 3.13666 & -1.13916\end{array}$

$\begin{array}{lll}5.23985 & 3.93249 & 0.80920\end{array}$

$\begin{array}{lll}6.28078 & 4.18533 & 1.02516\end{array}$

$\begin{array}{lll}4.24623 & 4.17270 & 1.75075\end{array}$

$\begin{array}{lll}4.51843 & 4.60772 & 2.71617\end{array}$

$\begin{array}{lll}2.90641 & 3.86686 & 1.49706\end{array}$

$\begin{array}{lll}3.26578 & 2.35588 & -2.03331\end{array}$

$\begin{array}{lll}2.20434 & 2.07847 & -2.00763\end{array}$

$\begin{array}{lll}4.06786 & 1.06916 & -2.23497\end{array}$

$\begin{array}{lll}3.91656 & 0.36256 & -1.40460\end{array}$

$\begin{array}{lll}3.76051 & 0.56561 & -3.16557\end{array}$

$\begin{array}{lll}5.14971 & 1.26384 & -2.31116\end{array}$

$\begin{array}{lll}3.46663 & 3.32035 & -3.20314\end{array}$

$\begin{array}{lll}4.51846 & 3.64167 & -3.28016\end{array}$

$\begin{array}{lll}3.19052 & 2.84234 & -4.15637\end{array}$

$\begin{array}{lll}2.84629 & 4.22289 & -3.09329\end{array}$

$\begin{array}{lll}1.89039 & 4.09390 & 2.60262\end{array}$

$\begin{array}{lll}0.89222 & 3.97294 & 2.15477\end{array}$

$\begin{array}{lll}2.03908 & 3.03243 & 3.69715\end{array}$

$\begin{array}{lll}3.02520 & 3.10331 & 4.18398\end{array}$

$\begin{array}{lll}1.26847 & 3.15625 & 4.47532\end{array}$

$\begin{array}{lll}1.94919 & 2.01249 & 3.28979\end{array}$

$\begin{array}{lll}1.94532 & 5.49910 & 3.20196\end{array}$

6.27184

$\begin{array}{lll}1.13885 & 5.63168 & 3.94076\end{array}$

$\begin{array}{lll}1.89693 & 5.63168 & 3.94076 \\ 2.896556 & 3.72489\end{array}$ 
[( ${ }^{\text {Dip }}$ LIn $\left.)_{2}\right]$, singlet, pbe0-gd3-bj/def2-tzvp 168

$\begin{array}{lllr}\text { In } & -1.14388 & 0.11710 & 1.02751 \\ \mathrm{P} & -3.54462 & 0.00583 & -0.66697 \\ \mathrm{~N} & -2.84069 & 1.27157 & 0.02211 \\ \mathrm{C} & -3.49155 & 0.04394 & -2.47997 \\ \mathrm{~N} & -2.65831 & -1.19128 & -0.06989 \\ \mathrm{C} & -2.25308 & -0.11482 & -3.11773 \\ \mathrm{H} & -1.34892 & -0.25487 & -2.52052 \\ \mathrm{C} & -2.16072 & -0.09218 & -4.50388 \\ \mathrm{H} & -1.18634 & -0.21915 & -4.98205\end{array}$

$\begin{array}{llll}\text { C } & -3.30839 & 0.08811 & -5.27796\end{array}$

$\begin{array}{llll}\mathrm{H} & -3.23823 & 0.10107 & -6.36824 \\ \mathrm{C} & -4.54311 & 0.25310 & -4.65552\end{array}$

$\begin{array}{llll}\mathrm{C} & -4.54311 & 0.25310 & -4.65552 \\ \mathrm{H} & -5.44475 & 0.39801 & -5.25514 \\ \mathrm{C} & -4.63626 & 0.23244 & -3.26368\end{array}$

$\begin{array}{lrrr}\mathrm{C} & -4.63626 & 0.23244 & -3.26368 \\ \mathrm{H} & -5.61015 & 0.35966 & -2.78832 \\ \mathrm{C} & -5.31333 & -0.19209 & -0.36221\end{array}$

$\begin{array}{llll}\mathrm{C} & -5.31333 & -0.19209 & -0.36221 \\ \mathrm{C} & -6.10952 & 0.89456 & 0.01259\end{array}$

$\begin{array}{llll}\mathrm{C} & -6.10952 & 0.89456 & 0.01259 \\ \mathrm{H} & -5.65665 & 1.87799 & 0.15012 \\ \mathrm{C} & -7.48222 & 0.72686 & 0.19013\end{array}$

$\begin{array}{llll}\mathrm{C} & -7.48222 & 0.72686 & 0.19013 \\ \mathrm{H} & -8.09858 & 1.57919 & 0.48563\end{array}$

$\begin{array}{rrrr}\mathrm{H} & -8.09858 & 1.57919 & 0.48563 \\ \mathrm{C} & -8.06608 & -0.52192 & -0.01689\end{array}$

$\begin{array}{lllr}\mathrm{C} & -8.06608 & -0.52192 & -0.01689 \\ \mathrm{H} & -9.14191 & -0.65196 & 0.12314 \\ \mathrm{C} & -7.27856 & -1.60426 & -0.41194\end{array}$

$\begin{array}{llll}\mathrm{C} & -7.27856 & -1.60426 & -0.41194 \\ \mathrm{H} & -7.73421 & -2.58245 & -0.58144\end{array}$

$\begin{array}{llll}\mathrm{H} & -7.73421 & -2.58245 & -0.58144 \\ \mathrm{C} & -5.90693 & -1.44250 & -0.58790 \\ \mathrm{H} & -5.29639 & -2.29259 & -0.89956 \\ \mathrm{C} & -3.05997 & 2.65560 & 0.09190\end{array}$

$\begin{array}{llll}\mathrm{C} & -3.05997 & 2.65560 & 0.09190 \\ \mathrm{C} & -2.52329 & 3.53959 & -0.87490\end{array}$

$\begin{array}{llll}\mathrm{C} & -2.52329 & 3.53959 & -0.87490 \\ \mathrm{C} & -2.72117 & 4.91566 & -0.72253\end{array}$

$\begin{array}{llll}\mathrm{C} & -2.72117 & 4.91566 & -0.72253 \\ \mathrm{H} & -2.31376 & 5.59422 & -1.47672 \\ \mathrm{C} & -3.40792 & 5.43676 & 0.36530\end{array}$

$\begin{array}{llll}\mathrm{C} & -3.40792 & 5.43676 & 0.36530 \\ \mathrm{H} & -3.55454 & 6.51493 & 0.46303\end{array}$

$\begin{array}{llll}\mathrm{H} & -3.55454 & 6.51493 & 0.46303 \\ \mathrm{C} & -3.88463 & 4.57048 & 1.34354\end{array}$

$\begin{array}{llll}\mathrm{C} & -3.88463 & 4.57048 & 1.34354 \\ \mathrm{H} & -4.39134 & 4.97986 & 2.22054 \\ \mathrm{C} & -3.71296 & 3.18917 & 1.23796\end{array}$

$\begin{array}{lllr}\mathrm{H} & -4.39134 & 4.97986 & 2.22054 \\ \mathrm{C} & -3.71296 & 3.18917 & 1.23796 \\ \mathrm{C} & -1.70598 & 3.04722 & -2.05030\end{array}$

$\begin{array}{llll}\mathrm{C} & -1.70598 & 3.04722 & -2.05030 \\ \mathrm{H} & -1.61172 & 1.95968 & -1.93800\end{array}$

$\begin{array}{llll}\mathrm{C} & -0.29060 & 3.62384 & -2.04266 \\ \mathrm{H} & -0.28964 & 4.72086 & -2.14005\end{array}$

$\begin{array}{lrrr}\mathrm{C} & -0.2806 & 3.62384 & -2.04266 \\ \mathrm{H} & 0.29380 & 3.72086 & -2.14005 \\ \mathrm{H} & 0.25014 & 3.3722 & -2.88430 \\ \mathrm{C} & -2.40719 & 3.31920 & -1.11639\end{array}$

$\begin{array}{lrrr}\mathrm{H} & 0.25014 & 3.37945 & -1.11639 \\ \mathrm{C} & -2.40719 & 3.31920 & -3.37923\end{array}$

$\begin{array}{llll}\mathrm{C} & -2.40719 & 3.31920 & -3.37923 \\ \mathrm{H} & -3.40479 & 2.85716 & -3.40802 \\ \mathrm{H} & -1.82305 & 2.91026 & -4.21785\end{array}$

$\begin{array}{llll}\mathrm{H} & -3.40479 & 2.85716 & -3.40802 \\ \mathrm{H} & -1.82305 & 2.91026 & -4.21785 \\ \mathrm{H} & -2.52985 & 4.40093 & -3.55066\end{array}$

$\begin{array}{llll}\mathrm{H} & -2.52985 & 4.40093 & -3.55066 \\ \mathrm{C} & -4.12962 & 2.29618 & 2.39210\end{array}$

$\begin{array}{llll}\mathrm{C} & -4.12962 & 2.29618 & 2.39210 \\ \mathrm{H} & -4.23383 & 1.27663 & 1.99458\end{array}$

$\begin{array}{llll}\mathrm{H} & -4.23383 & 1.27663 & 1.99458 \\ \mathrm{C} & -3.02543 & 2.26251 & 3.45152 \\ \mathrm{H} & -2.06552 & 1.92446 & 3.02845\end{array}$

$\begin{array}{llll}\mathrm{H} & -2.06552 & 1.92446 & 3.02845 \\ \mathrm{H} & -3.28810 & 1.58239 & 4.27749 \\ \mathrm{H} & -2.85804 & 3.26676 & 3.87249\end{array}$

$\begin{array}{llll}\mathrm{H} & -2.85804 & 3.26676 & 3.87249 \\ \mathrm{C} & -5.46255 & 2.68063 & 3.02798 \\ \mathrm{H} & -5.40272 & 3.64149 & 3.56285\end{array}$

$\begin{array}{llll}\mathrm{C} & -5.46255 & 2.68063 & 3.02798 \\ \mathrm{H} & -5.40272 & 3.64149 & 3.56285 \\ \mathrm{H} & -5.76565 & 1.91932 & 3.76348 \\ \mathrm{H} & -6.26494 & 2.76367 & 2.28039 \\ \mathrm{C} & -2.96946 & -2.51974 & 0.25423\end{array}$

$\begin{array}{llll}\mathrm{H} & -6.26494 & 2.76367 & 2.28039 \\ \mathrm{C} & -2.96946 & -2.51974 & 0.25423 \\ \mathrm{C} & -2.52918 & -3.57464 & -0.58169\end{array}$

$\begin{array}{llll}\mathrm{C} & -2.52918 & -2.574464 & -0.58169 \\ \mathrm{C} & -2.80908 & -4.89407 & -0.21722\end{array}$

$\begin{array}{llll}\mathrm{C} & -2.80908 & -4.89407 & -0.21722 \\ \mathrm{H} & -2.47490 & -5.70720 & -0.86691\end{array}$

$\begin{array}{llll}\mathrm{H} & -2.47490 & -5.70720 & -0.86691 \\ \mathrm{C} & -3.49341 & -5.19133 & 0.95558\end{array}$

$\begin{array}{llll}\mathrm{C} & -3.49341 & -5.19133 & 0.95558 \\ \mathrm{H} & -3.70464 & -6.22911 & 1.22404 \\ \mathrm{C} & -3.89066 & -4.15476 & 1.79323 \\ \mathrm{H} & -4.40552 & -4.38844 & 2.72886\end{array}$

$\begin{array}{llll}\mathrm{H} & -4.40552 & -4.38844 & 2.72886 \\ \mathrm{C} & -3.63589 & -2.81964 & 1.47184\end{array}$

$\begin{array}{lllr}\mathrm{C} & -3.63589 & -2.81964 & 1.47184 \\ \mathrm{C} & -1.77126 & -3.29077 & -1.86121\end{array}$

$\begin{array}{llll}\mathrm{C} & -1.77126 & -3.29077 & -1.86121 \\ \mathrm{H} & -1.50164 & -2.22636 & -1.82524 \\ \mathrm{C} & -0.47159 & -4.08605 & -1.97462\end{array}$

$\begin{array}{lrrr}\mathrm{C} & -0.47159 & -4.08605 & -1.97462 \\ \mathrm{H} & 0.19282 & -3.91413 & -1.11401\end{array}$

$\begin{array}{llll}\mathrm{H} & 0.19282 & -3.91413 & -1.11401 \\ \mathrm{H} & 0.07767 & -3.79140 & -2.88257\end{array}$

$\begin{array}{llll}\mathrm{H} & 0.07767 & -3.79140 & -2.88257 \\ \mathrm{H} & -0.65221 & -5.17061 & -2.04230 \\ \mathrm{C} & -2.66568 & -3.50282 & -3.08243 \\ \mathrm{H} & -2.98044 & -4.55626 & -3.16266 \\ \mathrm{H} & -2.13647 & -3.23377 & -4.01003\end{array}$

$\begin{array}{llll}\mathrm{H} & -2.98044 & -4.55626 & -3.16266 \\ \mathrm{H} & -2.13647 & -3.23377 & -4.01003 \\ \mathrm{H} & -3.57269 & -2.88184 & -3.02757\end{array}$

$\begin{array}{llll}\mathrm{H} & -2.13647 & -3.23377 & -4.01003 \\ \mathrm{H} & -3.57269 & -2.88184 & -3.02757 \\ \mathrm{C} & -4.00619 & -1.73584 & 2.46711 \\ \mathrm{H} & -3.89900 & -0.77009 & 1.95121 \\ \mathrm{C} & -3.03067 & -1.73713 & 3.64633\end{array}$

$\begin{array}{lll}-5.67730 & -0.96151 & 3.61181 \\ -5.63169 & -2.73336 & 3.54272\end{array}$


[ ${ }^{\text {Dip }}$ LTl:], singlet, pbe0/def2-tzvp 84

\begin{tabular}{|c|c|c|c|}
\hline $\mathrm{Tl}$ & 0.17254 & -0.59306 & -2.32720 \\
\hline $\mathrm{P}$ & -0.05970 & 0.44516 & 0.57650 \\
\hline $\mathrm{N}$ & 1.25125 & -0.08116 & -0.18060 \\
\hline $\mathrm{C}$ & 0.01006 & 2.24348 & 0.90364 \\
\hline $\mathrm{N}$ & -1.24552 & 0.11266 & -0.44924 \\
\hline $\mathrm{C}$ & -0.08696 & 3.12763 & -0.18155 \\
\hline $\mathrm{H}$ & -0.20812 & 2.72954 & -1.19272 \\
\hline $\mathrm{C}$ & -0.04044 & 4.50425 & 0.01031 \\
\hline $\mathrm{H}$ & -0.12252 & 5.17642 & -0.84753 \\
\hline $\mathrm{C}$ & 0.10646 & 5.02564 & 1.29766 \\
\hline $\mathrm{H}$ & 0.13926 & 6.10710 & 1.45108 \\
\hline $\mathrm{C}$ & 0.21091 & 4.16051 & 2.38315 \\
\hline $\mathrm{H}$ & 0.32754 & 4.55972 & 3.39373 \\
\hline $\mathrm{C}$ & 0.16307 & 2.77861 & 2.18836 \\
\hline $\mathrm{H}$ & 0.23933 & 2.11403 & 3.05099 \\
\hline $\mathrm{C}$ & -0.32949 & -0.21855 & 2.24834 \\
\hline $\mathrm{C}$ & 0.74272 & -0.69821 & 3.01180 \\
\hline $\mathrm{H}$ & 1.75038 & -0.70984 & 2.59185 \\
\hline $\mathrm{C}$ & 0.53492 & -1.15155 & 4.31438 \\
\hline $\mathrm{H}$ & 1.38016 & -1.52323 & 4.89897 \\
\hline $\mathrm{C}$ & -0.74359 & -1.12518 & 4.86908 \\
\hline $\mathrm{H}$ & -0.90607 & -1.48251 & 5.88911 \\
\hline $\mathrm{C}$ & -1.81383 & -0.63281 & 4.12207 \\
\hline $\mathrm{H}$ & -2.81718 & -0.60250 & 4.55363 \\
\hline $\mathrm{C}$ & -1.60991 & -0.17662 & 2.82124 \\
\hline $\mathrm{H}$ & -2.45636 & 0.21056 & 2.25115 \\
\hline $\mathrm{C}$ & 2.60865 & -0.23151 & 0.14398 \\
\hline $\mathrm{C}$ & 3.54977 & 0.81072 & -0.06368 \\
\hline $\mathrm{C}$ & 4.89472 & 0.59731 & 0.25806 \\
\hline $\mathrm{H}$ & 5.60928 & 1.41162 & 0.10784 \\
\hline $\mathrm{C}$ & 5.34431 & -0.61985 & 0.74940 \\
\hline $\mathrm{H}$ & 6.39764 & -0.76290 & 1.00230 \\
\hline $\mathrm{C}$ & 4.43692 & -1.66494 & 0.88751 \\
\hline $\mathrm{H}$ & 4.79349 & -2.63782 & 1.23373 \\
\hline $\mathrm{C}$ & 3.08402 & -1.50422 & 0.58124 \\
\hline $\mathrm{C}$ & 3.16310 & 2.13232 & -0.69732 \\
\hline $\mathrm{H}$ & 2.07540 & 2.11024 & -0.83488 \\
\hline $\mathrm{C}$ & 3.79413 & 2.27859 & -2.08477 \\
\hline $\mathrm{H}$ & 4.89400 & 2.31662 & -2.02797 \\
\hline $\mathrm{H}$ & 3.45458 & 3.20705 & -2.57225 \\
\hline $\mathrm{H}$ & 3.52912 & 1.43288 & -2.73888 \\
\hline $\mathrm{C}$ & 3.49180 & 3.33310 & 0.18800 \\
\hline $\mathrm{H}$ & 2.99599 & 3.25624 & 1.16699 \\
\hline $\mathrm{H}$ & 3.15536 & 4.26882 & -0.28555 \\
\hline $\mathrm{H}$ & 4.57605 & 3.42292 & 0.36443 \\
\hline $\mathrm{C}$ & 2.16270 & -2.71070 & 0.62723 \\
\hline $\mathrm{H}$ & 1.13926 & -2.33300 & 0.76443 \\
\hline $\mathrm{C}$ & 2.20387 & -3.44824 & -0.71503 \\
\hline $\mathrm{H}$ & 1.96456 & -2.77797 & -1.55632 \\
\hline $\mathrm{H}$ & 1.48858 & -4.28659 & -0.73381 \\
\hline $\mathrm{H}$ & 3.20994 & -3.85481 & -0.90680 \\
\hline $\mathrm{C}$ & 2.45058 & -3.68167 & 1.77025 \\
\hline $\mathrm{H}$ & 3.41513 & -4.19850 & 1.64376 \\
\hline $\mathrm{H}$ & 1.67303 & -4.46037 & 1.81203 \\
\hline $\mathrm{H}$ & 2.46691 & -3.17266 & 2.74547 \\
\hline $\mathrm{C}$ & -2.57540 & -0.28534 & -0.24120 \\
\hline $\mathrm{C}$ & -3.63818 & 0.65216 & -0.34438 \\
\hline $\mathrm{C}$ & -4.95457 & 0.22660 & -0.14403 \\
\hline $\mathrm{H}$ & -5.76452 & 0.95866 & -0.21139 \\
\hline $\mathrm{C}$ & -5.25727 & -1.10096 & 0.13244 \\
\hline $\mathrm{H}$ & -6.29240 & -1.41396 & 0.28913 \\
\hline $\mathrm{C}$ & -4.22328 & -2.02777 & 0.19005 \\
\hline $\mathrm{H}$ & -4.45724 & -3.07826 & 0.38422 \\
\hline $\mathrm{C}$ & -2.89004 & -1.65214 & 0.00414 \\
\hline $\mathrm{C}$ & -3.37945 & 2.11057 & -0.66532 \\
\hline $\mathrm{H}$ & -2.30985 & 2.18711 & -0.89826 \\
\hline $\mathrm{C}$ & -4.15364 & 2.57595 & -1.89930 \\
\hline $\mathrm{H}$ & -3.94081 & 1.93811 & -2.77111 \\
\hline $\mathrm{H}$ & -3.88146 & 3.61162 & -2.15957 \\
\hline $\mathrm{H}$ & -5.24296 & 2.55713 & -1.73377 \\
\hline $\mathrm{C}$ & -3.65473 & 3.01645 & 0.53571 \\
\hline $\mathrm{H}$ & -4.71611 & 2.97898 & 0.83179 \\
\hline $\mathrm{H}$ & -3.40755 & 4.06434 & 0.30157 \\
\hline $\mathrm{H}$ & -3.05156 & 2.72197 & 1.40801 \\
\hline $\mathrm{C}$ & -1.82414 & -2.73270 & 0.01791 \\
\hline $\mathrm{H}$ & -0.84927 & -2.22136 & 0.00340 \\
\hline $\mathrm{C}$ & -1.91582 & -3.59737 & -1.24342 \\
\hline $\mathrm{H}$ & -2.87042 & -4.14662 & -1.27890 \\
\hline $\mathrm{H}$ & -1.10057 & -4.33811 & -1.27798 \\
\hline $\mathrm{H}$ & -1.86385 & -2.98847 & -2.16083 \\
\hline $\mathrm{C}$ & -1.85532 & -3.60018 & 1.27601 \\
\hline $\mathrm{H}$ & -1.76456 & -2.98924 & 2.18603 \\
\hline $\mathrm{H}$ & -1.02306 & -4.32212 & 1.26612 \\
\hline $\mathrm{H}$ & -2.78867 & -4.18098 & 1.34951 \\
\hline
\end{tabular}

[ ${ }^{\text {Dip }}$ LTl:], singlet, pbe0-gd3-bj/def2-tzvp 84

$\begin{array}{lrcc}\mathrm{T} 1 & 0.23099 & -0.45248 & -2.40736 \\ \mathrm{P} & -0.08396 & 0.42139 & 0.55909 \\ \mathrm{~N} & 1.22703 & -0.13334 & -0.17406 \\ \mathrm{C} & 0.04745 & 2.19130 & 0.96171\end{array}$

$\begin{array}{lll}-1.24784 & 0.19164 & -0.51387\end{array}$

$\begin{array}{lll}-0.07237 & 3.12504 & -0.07727\end{array}$

$\begin{array}{lll}-0.24620 & 2.77461 & -1.09790\end{array}$

$\begin{array}{lll}0.01201 & 4.48833 & 0.17891\end{array}$

$\begin{array}{lll}-0.08854 & 5.20371 & -0.64084\end{array}$

$\begin{array}{lll}0.21774 & 4.94183 & 1.48378\end{array}$

$\begin{array}{lll}0.27871 & 6.01344 & 1.68769\end{array}$

$\begin{array}{lll}0.34371 & 4.02389 & 2.52268\end{array}$

$\begin{array}{lll}0.50525 & 4.37228 & 3.54551\end{array}$

$\begin{array}{lll}0.26022 & 2.65491 & 2.26412\end{array}$

$\begin{array}{lll}0.35024 & 1.94345 & 3.08715\end{array}$

$\begin{array}{lll}-0.43312 & -0.30030 & 2.18478 \\ 0.60928 & -0.81633 & 2.96509\end{array}$

$\begin{array}{lll}0.60928 & -0.81633 & 2.96509\end{array}$

$\begin{array}{lll}0.63305 & -0.80116 & 2.58712\end{array}$

$\begin{array}{lll}0.34886 & -1.34086 & 4.22974\end{array}$

$\begin{array}{lll}1.16990 & -1.74331 & 4.82783\end{array}$

$\begin{array}{lll}-0.95265 & -1.34839 & 4.72903\end{array}$

$\begin{array}{lll}-1.15719 & -1.76488 & 5.71835\end{array}$

$\begin{array}{lll}-1.99143 & -0.81297 & 3.96803\end{array}$

$\begin{array}{lll}-3.01212 & -0.80750 & 4.35706\end{array}$

$\begin{array}{lll}-1.73547 & -0.28418 & 2.70514\end{array}$

$\begin{array}{lll}-2.55863 & 0.13200 & 2.12327\end{array}$

$\begin{array}{lll}2.56626 & -0.27788 & 0.20675\end{array}$

$\begin{array}{lll}3.50689 & 0.76705 & 0.03718\end{array}$

$\begin{array}{lll}4.83601 & 0.55983 & 0.42014\end{array}$

$\begin{array}{lll}5.55507 & 1.37470 & 0.29879 \\ 5.26248 & -0.65509 & 0.93841\end{array}$

6.30308

$\begin{array}{lll}6.35277 & -1.70332 & 1.04330\end{array}$

$\begin{array}{lll}4.69417 & -2.67206 & 1.41447\end{array}$

$\begin{array}{lll}3.01662 & -1.54554 & 0.67346\end{array}$

$\begin{array}{lll}3.13041 & 2.07391 & -0.62697\end{array}$

$\begin{array}{lll}2.04508 & 2.04837 & -0.77964\end{array}$

$\begin{array}{lll}3.78044 & 2.18324 & -2.00732\end{array}$

$\begin{array}{lll}4.87943 & 2.21562 & -1.93470\end{array}$

$\begin{array}{lll}3.45186 & 3.09980 & -2.52365\end{array}$

$\begin{array}{lll}3.52012 & 1.32053 & -2.64096\end{array}$

$\begin{array}{lll}3.44275 & 3.29190 & 0.23658\end{array}$

$\begin{array}{lll}2.93180 & 3.23020 & 1.20836\end{array}$

$\begin{array}{lll}3.10812 & 4.21591 & -0.25986\end{array}$

$\begin{array}{lll}4.52389 & 3.38913 & 0.42620\end{array}$

$\begin{array}{lll}2.07631 & -2.73523 & 0.68532\end{array}$

$\begin{array}{lll}1.06057 & -2.34482 & 0.83694\end{array}$

$\begin{array}{lll}2.09511 & -3.42435 & -0.68024\end{array}$

$\begin{array}{lll}1.84676 & -2.71889 & -1.48878\end{array}$

$\begin{array}{lll}1.876767 & -4.25398 & -0.71888 \\ 3.09496 & -3.83074 & -0.90044\end{array}$

$\begin{array}{lll}3.09496 & -3.83074 & -0.90044\end{array}$

$\begin{array}{lll}2.35075 & -3.74251 & 1.79632\end{array}$

$\begin{array}{lll}3.30472 & -4.27327 & 1.64998\end{array}$

$\begin{array}{lll}.55730 & -4.50505 & 1.82079\end{array}$

$\begin{array}{lll}2.38160 & -3.25943 & 2.78428\end{array}$

$\begin{array}{lll}-2.55842 & -0.25903 & -0.31464\end{array}$

$\begin{array}{lll}-3.63879 & 0.65904 & -0.34059 \\ -4.93893 & 0.19399 & -0.12953\end{array}$

$-5.76725$

$\begin{array}{lll}-5.19962 & -1.15433 & 0.08939\end{array}$

$\begin{array}{lll}-6.22225 & -1.50005 & 0.25769\end{array}$

$\begin{array}{lll}-4.14376 & -2.05854 & 0.08059\end{array}$

$\begin{array}{lll}-4.34697 & -3.12137 & 0.23785\end{array}$

$\begin{array}{lll}-2.82640 & -1.64029 & -0.12124\end{array}$

$\begin{array}{lll}-3.38801 & 2.13731 & -0.54664\end{array}$

$\begin{array}{lll}-2.35770 & 2.21863 & -0.91874\end{array}$

$\begin{array}{lll}-4.31256 & 2.75676 & -1.59122\end{array}$

$\begin{array}{lll}-4.26837 & 2.20566 & -2.54299\end{array}$

$\begin{array}{lll}-4.02569 & 3.80218 & -1.78684\end{array}$

$\begin{array}{lll}-5.36342 & 2.76658 & -1.26019\end{array}$

$\begin{array}{lll}-3.45797 & 2.89834 & 0.77740\end{array}$

$\begin{array}{lll}-4.46821 & 2.83701 & 1.21440\end{array}$

$\begin{array}{lll}-3.21040 & 3.96228 & 0.63536\end{array}$

$\begin{array}{lll}-2.74680 & 2.49260 & 1.51180\end{array}$

$\begin{array}{lll}-1.72219 & -2.67808 & -0.16756\end{array}$

$\begin{array}{lll}-0.76662 & -2.13173 & -0.18113\end{array}$

$\begin{array}{lll}-1.80838 & -3.49333 & -1.45971\end{array}$

$\begin{array}{lll}-2.74247 & -4.07606 & -1.49853\end{array}$

$\begin{array}{lll}-0.96596 & -4.19824 & -1.54279\end{array}$

$\begin{array}{lll}-1.80104 & -2.84320 & -2.34982\end{array}$

$\begin{array}{lll}-1.69429 & -3.58865 & 1.05756\end{array}$

$\begin{array}{lll}-1.58568 & -3.00732 & 1.98437\end{array}$

$\begin{array}{lll}-0.84794 & -4.29069 & 0.99493\end{array}$

$\begin{array}{lll}-2.61293 & -4.19079 & 1.14017\end{array}$ 
[ ${ }^{\text {Dip }}$ LT1:], triplet, pbe0/def2-tzvp 84

\begin{tabular}{|c|c|c|c|}
\hline $\mathrm{Tl}$ & -0.32092 & -1.62069 & -2.11047 \\
\hline $\mathrm{P}$ & 0.06210 & 0.18184 & 0.89445 \\
\hline $\mathrm{N}$ & -1.10915 & 0.07230 & -0.12901 \\
\hline $\mathrm{C}$ & 0.19820 & -1.10212 & 2.17631 \\
\hline $\mathrm{N}$ & 1.44307 & -0.13584 & 0.04471 \\
\hline C & 0.43559 & -2.43022 & 1.77710 \\
\hline $\mathrm{H}$ & 0.57977 & -2.65968 & 0.71685 \\
\hline C & 0.44862 & -3.45822 & 2.71555 \\
\hline $\mathrm{H}$ & 0.62463 & -4.48551 & 2.38688 \\
\hline C & 0.24533 & -3.17834 & 4.06718 \\
\hline $\mathrm{H}$ & 0.26978 & -3.98495 & 4.80436 \\
\hline $\mathrm{C}$ & 0.01005 & -1.86536 & 4.47465 \\
\hline $\mathrm{H}$ & -0.15669 & -1.63918 & 5.53056 \\
\hline $\mathrm{C}$ & -0.02088 & -0.83538 & 3.53582 \\
\hline $\mathrm{H}$ & -0.22074 & 0.18393 & 3.87023 \\
\hline $\mathrm{C}$ & 0.13080 & 1.70982 & 1.86873 \\
\hline C & -0.98966 & 2.54460 & 1.95386 \\
\hline $\mathrm{H}$ & -1.89508 & 2.29629 & 1.39589 \\
\hline $\mathrm{C}$ & -0.95472 & 3.68359 & 2.75889 \\
\hline $\mathrm{H}$ & -1.83537 & 4.32714 & 2.82346 \\
\hline C & 0.19538 & 3.99496 & 3.48310 \\
\hline $\mathrm{H}$ & 0.21950 & 4.88629 & 4.11495 \\
\hline C & 1.31519 & 3.16506 & 3.40444 \\
\hline $\mathrm{H}$ & 2.21693 & 3.40511 & 3.97313 \\
\hline C & 1.28418 & 2.02646 & 2.60277 \\
\hline $\mathrm{H}$ & 2.15990 & 1.37387 & 2.55719 \\
\hline $\mathrm{C}$ & -2.47761 & 0.36473 & -0.20520 \\
\hline C & -3.45194 & -0.44239 & 0.44387 \\
\hline $\mathrm{C}$ & -4.80583 & -0.11497 & 0.32242 \\
\hline $\mathrm{H}$ & -5.54508 & -0.73417 & 0.83847 \\
\hline $\mathrm{C}$ & -5.23413 & 0.96379 & -0.43957 \\
\hline $\mathrm{H}$ & -6.29736 & 1.20286 & -0.51896 \\
\hline $\mathrm{C}$ & -4.28582 & 1.71956 & -1.11719 \\
\hline $\mathrm{H}$ & -4.61404 & 2.55248 & -1.74547 \\
\hline $\mathrm{C}$ & -2.91947 & 1.43981 & -1.02826 \\
\hline C & -3.08105 & -1.66435 & 1.25994 \\
\hline $\mathrm{H}$ & -1.99266 & -1.77973 & 1.19461 \\
\hline C & -3.69264 & -2.94245 & 0.68283 \\
\hline $\mathrm{H}$ & -4.79403 & -2.92393 & 0.71921 \\
\hline $\mathrm{H}$ & -3.35517 & -3.82231 & 1.25379 \\
\hline $\mathrm{H}$ & -3.39212 & -3.08822 & -0.36610 \\
\hline C & -3.44697 & -1.49660 & 2.73571 \\
\hline $\mathrm{H}$ & -3.00288 & -0.58347 & 3.16028 \\
\hline $\mathrm{H}$ & -3.08626 & -2.35268 & 3.32765 \\
\hline $\mathrm{H}$ & -4.53865 & -1.42875 & 2.87370 \\
\hline $\mathrm{C}$ & -1.95917 & 2.28135 & -1.84783 \\
\hline $\mathrm{H}$ & -0.94870 & 1.91286 & -1.62191 \\
\hline C & -2.18990 & 2.08147 & -3.34747 \\
\hline $\mathrm{H}$ & -2.08855 & 1.02088 & -3.62407 \\
\hline $\mathrm{H}$ & -1.45209 & 2.65121 & -3.93548 \\
\hline $\mathrm{H}$ & -3.19361 & 2.42115 & -3.65107 \\
\hline C & -2.02725 & 3.76615 & -1.48381 \\
\hline $\mathrm{H}$ & -3.02510 & 4.18704 & -1.68706 \\
\hline $\mathrm{H}$ & -1.30529 & 4.34828 & -2.07924 \\
\hline $\mathrm{H}$ & -1.80408 & 3.94021 & -0.42039 \\
\hline C & 2.61480 & 0.40942 & -0.25416 \\
\hline C & 3.73864 & -0.50900 & -0.36611 \\
\hline $\mathrm{C}$ & 5.02273 & 0.00919 & -0.45295 \\
\hline $\mathrm{H}$ & 5.87570 & -0.67340 & -0.47285 \\
\hline $\mathrm{C}$ & 5.24742 & 1.38600 & -0.51090 \\
\hline $\mathrm{H}$ & 6.26870 & 1.77074 & -0.56838 \\
\hline $\mathrm{C}$ & 4.16645 & 2.27089 & -0.55879 \\
\hline $\mathrm{H}$ & 4.35886 & 3.33530 & -0.70971 \\
\hline $\mathrm{C}$ & 2.85540 & 1.82507 & -0.47850 \\
\hline C & 3.52660 & -2.00298 & -0.28635 \\
\hline $\mathrm{H}$ & 2.44800 & -2.17682 & -0.42243 \\
\hline $\mathrm{C}$ & 4.26445 & -2.75023 & -1.39742 \\
\hline $\mathrm{H}$ & 3.99716 & -2.36262 & -2.39220 \\
\hline $\mathrm{H}$ & 4.00048 & -3.81861 & -1.36967 \\
\hline $\mathrm{H}$ & 5.35900 & -2.68245 & -1.29098 \\
\hline C & 3.91121 & -2.53763 & 1.09581 \\
\hline $\mathrm{H}$ & 4.98787 & -2.39898 & 1.28825 \\
\hline $\mathrm{H}$ & 3.69143 & -3.61432 & 1.16848 \\
\hline $\mathrm{H}$ & 3.35283 & -2.02820 & 1.89460 \\
\hline C & 1.72605 & 2.75508 & -0.85454 \\
\hline $\mathrm{H}$ & 0.78731 & 2.32270 & -0.47981 \\
\hline $\mathrm{C}$ & 1.62420 & 2.78482 & -2.38681 \\
\hline $\mathrm{H}$ & 2.55305 & 3.17649 & -2.83153 \\
\hline $\mathrm{H}$ & 0.79413 & 3.43380 & -2.70336 \\
\hline $\mathrm{H}$ & 1.44220 & 1.78067 & -2.79995 \\
\hline $\mathrm{C}$ & 1.85240 & 4.16440 & -0.28465 \\
\hline $\mathrm{H}$ & 1.95932 & 4.15260 & 0.80953 \\
\hline $\mathrm{H}$ & 0.95168 & 4.74638 & -0.53024 \\
\hline $\mathrm{H}$ & 2.71124 & 4.70708 & -0.71078 \\
\hline
\end{tabular}


[( $\left.{ }^{\text {Dip }} \mathbf{L T I}\right)_{2}$ ], singlet, pbe0/def2-tzvp 168

\begin{tabular}{|c|c|c|c|}
\hline $\mathrm{Tl}$ & 1.49749 & -0.50682 & 1.05325 \\
\hline $\mathrm{P}$ & 3.98645 & 0.14035 & -0.63177 \\
\hline $\mathrm{N}$ & 3.58582 & -1.25353 & 0.05926 \\
\hline $\mathrm{C}$ & 3.86100 & 0.05357 & -2.45378 \\
\hline $\mathrm{N}$ & 2.90691 & 1.16636 & -0.04501 \\
\hline $\mathrm{C}$ & 2.58644 & -0.05908 & -3.03047 \\
\hline $\mathrm{H}$ & 1.70125 & -0.08956 & -2.38879 \\
\hline C & 2.42935 & -0.12408 & -4.41073 \\
\hline $\mathrm{H}$ & 1.42779 & -0.20799 & -4.84030 \\
\hline $\mathrm{C}$ & 3.54864 & -0.07737 & -5.24487 \\
\hline $\mathrm{H}$ & 3.42679 & -0.12437 & -6.32981 \\
\hline $\mathrm{C}$ & 4.81918 & 0.02974 & -4.68635 \\
\hline $\mathrm{H}$ & 5.70066 & 0.06682 & -5.33120 \\
\hline $\mathrm{C}$ & 4.97512 & 0.09499 & -3.30037 \\
\hline $\mathrm{H}$ & 5.97783 & 0.18695 & -2.87947 \\
\hline $\mathrm{C}$ & 5.71675 & 0.65339 & -0.40459 \\
\hline $\mathrm{C}$ & 6.72963 & -0.30972 & -0.29348 \\
\hline $\mathrm{H}$ & 6.47628 & -1.37202 & -0.28586 \\
\hline $\mathrm{C}$ & 8.06560 & 0.07835 & -0.20263 \\
\hline $\mathrm{H}$ & 8.84529 & -0.68263 & -0.11721 \\
\hline $\mathrm{C}$ & 8.40506 & 1.43053 & -0.22578 \\
\hline $\mathrm{H}$ & 9.45260 & 1.73391 & -0.15395 \\
\hline $\mathrm{C}$ & 7.40501 & 2.39435 & -0.34893 \\
\hline $\mathrm{H}$ & 7.66467 & 3.45542 & -0.37466 \\
\hline $\mathrm{C}$ & 6.06801 & 2.01077 & -0.44395 \\
\hline $\mathrm{H}$ & 5.29722 & 2.77639 & -0.54786 \\
\hline $\mathrm{C}$ & 4.16152 & -2.53366 & 0.10898 \\
\hline $\mathrm{C}$ & 3.83660 & -3.53754 & -0.83991 \\
\hline $\mathrm{C}$ & 4.42594 & -4.80199 & -0.73045 \\
\hline $\mathrm{H}$ & 4.18083 & -5.56543 & -1.47425 \\
\hline $\mathrm{C}$ & 5.30063 & -5.11313 & 0.30088 \\
\hline $\mathrm{H}$ & 5.75579 & -6.10455 & 0.36335 \\
\hline C & 5.56806 & -4.15177 & 1.27006 \\
\hline $\mathrm{H}$ & 6.22561 & -4.40489 & 2.10493 \\
\hline $\mathrm{C}$ & 5.00700 & -2.87470 & 1.20632 \\
\hline $\mathrm{C}$ & 2.81204 & -3.31173 & -1.93387 \\
\hline $\mathrm{H}$ & 2.50550 & -2.26056 & -1.86877 \\
\hline $\mathrm{C}$ & 1.56256 & -4.16460 & -1.69691 \\
\hline $\mathrm{H}$ & 1.78782 & -5.24167 & -1.75538 \\
\hline $\mathrm{H}$ & 0.79045 & -3.94575 & -2.45262 \\
\hline $\mathrm{H}$ & 1.12918 & -3.97514 & -0.70202 \\
\hline $\mathrm{C}$ & 3.38114 & -3.53960 & -3.33307 \\
\hline $\mathrm{H}$ & 4.24736 & -2.88833 & -3.52262 \\
\hline $\mathrm{H}$ & 2.62299 & -3.32138 & -4.10156 \\
\hline $\mathrm{H}$ & 3.70463 & -4.58358 & -3.47504 \\
\hline $\mathrm{C}$ & 5.23449 & -1.90117 & 2.34918 \\
\hline $\mathrm{H}$ & 5.10026 & -0.88856 & 1.94277 \\
\hline $\mathrm{C}$ & 4.16834 & -2.10772 & 3.42983 \\
\hline $\mathrm{H}$ & 3.14945 & -2.02282 & 3.01907 \\
\hline $\mathrm{H}$ & 4.27164 & -1.36692 & 4.23927 \\
\hline $\mathrm{H}$ & 4.25308 & -3.11219 & 3.87480 \\
\hline $\mathrm{C}$ & 6.63021 & -1.96715 & 2.96477 \\
\hline $\mathrm{H}$ & 6.80283 & -2.91260 & 3.50327 \\
\hline $\mathrm{H}$ & 6.76174 & -1.15364 & 3.69524 \\
\hline $\mathrm{H}$ & 7.41750 & -1.86335 & 2.20341 \\
\hline $\mathrm{C}$ & 2.96503 & 2.52190 & 0.31538 \\
\hline $\mathrm{C}$ & 2.50110 & 3.53142 & -0.57109 \\
\hline $\mathrm{C}$ & 2.55131 & 4.87089 & -0.17376 \\
\hline $\mathrm{H}$ & 2.20393 & 5.64184 & -0.86683 \\
\hline $\mathrm{C}$ & 3.02307 & 5.24311 & 1.07909 \\
\hline $\mathrm{H}$ & 3.05486 & 6.29582 & 1.37028 \\
\hline C & 3.43879 & 4.25399 & 1.96213 \\
\hline $\mathrm{H}$ & 3.79048 & 4.53913 & 2.95753 \\
\hline C & 3.41705 & 2.90134 & 1.61115 \\
\hline $\mathrm{C}$ & 1.96924 & 3.19198 & -1.94917 \\
\hline $\mathrm{H}$ & 1.82900 & 2.10268 & -1.96353 \\
\hline $\mathrm{C}$ & 0.61379 & 3.83742 & -2.23750 \\
\hline $\mathrm{H}$ & -0.13220 & 3.60000 & -1.46342 \\
\hline $\mathrm{H}$ & 0.22115 & 3.48611 & -3.20521 \\
\hline $\mathrm{H}$ & 0.68241 & 4.93521 & -2.30039 \\
\hline $\mathrm{C}$ & 2.98593 & 3.54033 & -3.03828 \\
\hline $\mathrm{H}$ & 3.17482 & 4.62617 & -3.06961 \\
\hline $\mathrm{H}$ & 2.61977 & 3.23187 & -4.03054 \\
\hline $\mathrm{H}$ & 3.94965 & 3.03651 & -2.86977 \\
\hline $\mathrm{C}$ & 3.83976 & 1.87817 & 2.65017 \\
\hline $\mathrm{H}$ & 3.88584 & 0.90271 & 2.14126 \\
\hline $\mathrm{C}$ & 2.79755 & 1.77345 & 3.76804 \\
\hline $\mathrm{H}$ & 2.72096 & 2.71854 & 4.32949 \\
\hline $\mathrm{H}$ & 3.06190 & 0.97817 & 4.48372 \\
\hline $\mathrm{H}$ & 1.79258 & 1.55733 & 3.37025 \\
\hline $\mathrm{C}$ & 5.23018 & 2.14460 & 3.22754 \\
\hline $\mathrm{H}$ & 5.99039 & 2.19392 & 2.43438 \\
\hline $\mathrm{H}$ & 5.51816 & 1.34190 & 3.92533 \\
\hline $\mathrm{H}$ & 5.26555 & 3.09194 & 3.78896 \\
\hline
\end{tabular}

\section{(continued)}

\begin{tabular}{|c|c|c|c|}
\hline $\mathrm{Tl}$ & -1.49776 & -0.50650 & -1.05390 \\
\hline$P$ & -3.98626 & 0.14021 & 0.63198 \\
\hline $\mathrm{N}$ & -2.90696 & 1.16643 & 0.04519 \\
\hline $\mathrm{C}$ & -3.86006 & 0.05267 & 2.45390 \\
\hline $\mathrm{N}$ & -3.58592 & -1.25343 & -0.05975 \\
\hline $\mathrm{C}$ & -2.58522 & -0.05976 & 3.03003 \\
\hline $\mathrm{H}$ & -1.70028 & -0.08962 & 2.38799 \\
\hline C & -2.42756 & -0.12532 & 4.41021 \\
\hline $\mathrm{H}$ & -1.42579 & -0.20903 & 4.83933 \\
\hline $\mathrm{C}$ & -3.54653 & -0.07942 & 5.24481 \\
\hline $\mathrm{H}$ & -3.42423 & -0.12685 & 6.32968 \\
\hline $\mathrm{C}$ & -4.81733 & 0.02745 & 4.68685 \\
\hline $\mathrm{H}$ & -5.69857 & 0.06391 & 5.33206 \\
\hline $\mathrm{C}$ & -4.97385 & 0.09327 & 3.30095 \\
\hline $\mathrm{H}$ & -5.97676 & 0.18505 & 2.88050 \\
\hline C & -5.71667 & 0.65334 & 0.40574 \\
\hline $\mathrm{C}$ & -6.06791 & 2.01072 & 0.44555 \\
\hline $\mathrm{H}$ & -5.29708 & 2.77632 & 0.54923 \\
\hline C & -7.40497 & 2.39431 & 0.35127 \\
\hline $\mathrm{H}$ & -7.66460 & 3.45538 & 0.37733 \\
\hline $\mathrm{C}$ & -8.40507 & 1.43052 & 0.22841 \\
\hline $\mathrm{H}$ & -9.45264 & 1.73392 & 0.15714 \\
\hline $\mathrm{C}$ & -8.06562 & 0.07835 & 0.20481 \\
\hline $\mathrm{H}$ & -8.84535 & -0.68263 & 0.11961 \\
\hline $\mathrm{C}$ & -6.72961 & -0.30974 & 0.29494 \\
\hline $\mathrm{H}$ & -6.47626 & -1.37204 & 0.28695 \\
\hline C & -2.96525 & 2.52197 & -0.31513 \\
\hline C & -2.50119 & 3.53148 & 0.57128 \\
\hline $\mathrm{C}$ & -2.55148 & 4.87096 & 0.17397 \\
\hline $\mathrm{H}$ & -2.20401 & 5.64191 & 0.86700 \\
\hline $\mathrm{C}$ & -3.02346 & 5.24319 & -1.07879 \\
\hline $\mathrm{H}$ & -3.05532 & 6.29590 & -1.36996 \\
\hline $\mathrm{C}$ & -3.43933 & 4.25407 & -1.96177 \\
\hline $\mathrm{H}$ & -3.79119 & 4.53923 & -2.95712 \\
\hline C & -3.41752 & 2.90142 & -1.61082 \\
\hline C & -1.96919 & 3.19202 & 1.94930 \\
\hline $\mathrm{H}$ & -1.82876 & 2.10273 & 1.96355 \\
\hline C & -0.61386 & 3.83769 & 2.23765 \\
\hline $\mathrm{H}$ & -0.68267 & 4.93546 & 2.30066 \\
\hline $\mathrm{H}$ & -0.22112 & 3.48638 & 3.20533 \\
\hline $\mathrm{H}$ & 0.13217 & 3.60047 & 1.46354 \\
\hline C & -2.98591 & 3.54009 & 3.03848 \\
\hline $\mathrm{H}$ & -3.94954 & 3.03608 & 2.86999 \\
\hline $\mathrm{H}$ & -2.61964 & 3.23165 & 4.03070 \\
\hline $\mathrm{H}$ & -3.17501 & 4.62589 & 3.06987 \\
\hline C & -3.84038 & 1.87826 & -2.64979 \\
\hline $\mathrm{H}$ & -3.88628 & 0.90278 & -2.14091 \\
\hline C & -2.79841 & 1.77365 & -3.76790 \\
\hline $\mathrm{H}$ & -1.79330 & 1.55778 & -3.37033 \\
\hline $\mathrm{H}$ & -3.06278 & 0.97825 & -4.48344 \\
\hline $\mathrm{H}$ & -2.72214 & 2.71870 & -4.32946 \\
\hline C & -5.23094 & 2.14462 & -3.22685 \\
\hline $\mathrm{H}$ & -5.26649 & 3.09201 & -3.78817 \\
\hline $\mathrm{H}$ & -5.51900 & 1.34196 & -3.92466 \\
\hline $\mathrm{H}$ & -5.99100 & 2.19381 & -2.43353 \\
\hline C & -4.16167 & -2.53354 & -0.10939 \\
\hline $\mathrm{C}$ & -3.83609 & -3.53770 & 0.83898 \\
\hline C & -4.42561 & -4.80207 & 0.72963 \\
\hline $\mathrm{H}$ & -4.17998 & -5.56574 & 1.47303 \\
\hline $\mathrm{C}$ & -5.30111 & -5.11286 & -0.30111 \\
\hline $\mathrm{H}$ & -5.75641 & -6.10423 & -0.36349 \\
\hline $\mathrm{C}$ & -5.56918 & -4.15123 & -1.26986 \\
\hline $\mathrm{H}$ & -6.22738 & -4.40409 & -2.10430 \\
\hline $\mathrm{C}$ & -5.00795 & -2.87424 & -1.20621 \\
\hline C & -2.81061 & -3.31230 & 1.93217 \\
\hline $\mathrm{H}$ & -2.50423 & -2.26106 & 1.86733 \\
\hline C & -1.56126 & -4.16493 & 1.69365 \\
\hline $\mathrm{H}$ & -1.12878 & -3.97488 & 0.69847 \\
\hline $\mathrm{H}$ & -0.78850 & -3.94645 & 2.44880 \\
\hline $\mathrm{H}$ & -1.78639 & -5.24205 & 1.75172 \\
\hline C & -3.37843 & -3.54092 & 3.33176 \\
\hline $\mathrm{H}$ & -3.70171 & -4.58500 & 3.47350 \\
\hline $\mathrm{H}$ & -2.61958 & -3.32305 & 4.09966 \\
\hline $\mathrm{H}$ & -4.24452 & -2.88980 & 3.52243 \\
\hline C & -5.23610 & -1.90041 & -2.34868 \\
\hline $\mathrm{H}$ & -5.10177 & -0.88789 & -1.94207 \\
\hline C & -4.17041 & -2.10656 & -3.42987 \\
\hline $\mathrm{H}$ & -4.25523 & -3.11093 & -3.87505 \\
\hline $\mathrm{H}$ & -4.27416 & -1.36557 & -4.23909 \\
\hline $\mathrm{H}$ & -3.15137 & -2.02162 & -3.01953 \\
\hline $\mathrm{C}$ & -6.63210 & -1.96635 & -2.96364 \\
\hline $\mathrm{H}$ & -7.41904 & -1.86293 & -2.20187 \\
\hline $\mathrm{H}$ & -6.76408 & -1.15260 & -3.69376 \\
\hline $\mathrm{H}$ & -6.80485 & -2.91163 & -3.50239 \\
\hline
\end{tabular}


[( $\left.\left.{ }^{\text {Dip }} \mathbf{L T I}\right)_{2}\right]$, singlet, pbe0-gd3-bj/def2-tzvp 168

\begin{tabular}{|c|c|c|c|}
\hline $\mathrm{Tl}$ & -1.14384 & -0.39740 & -1.17157 \\
\hline $\mathrm{P}$ & -3.54953 & 0.11763 & 0.66614 \\
\hline $\mathrm{N}$ & -3.07440 & -1.25460 & -0.00698 \\
\hline C & -3.51743 & 0.06965 & 2.48308 \\
\hline $\mathrm{N}$ & -2.49716 & 1.18422 & 0.10070 \\
\hline $\mathrm{C}$ & -2.27777 & 0.11668 & 3.13526 \\
\hline $\mathrm{H}$ & -1.36242 & 0.17311 & 2.54310 \\
\hline $\mathrm{C}$ & -2.20099 & 0.09753 & 4.52238 \\
\hline $\mathrm{H}$ & -1.22503 & 0.13982 & 5.01234 \\
\hline $\mathrm{C}$ & -3.36912 & 0.03195 & 5.28446 \\
\hline $\mathrm{H}$ & -3.31231 & 0.02324 & 6.37550 \\
\hline $\mathrm{C}$ & -4.60661 & -0.02253 & 4.64842 \\
\hline $\mathrm{H}$ & -5.52440 & -0.07599 & 5.23865 \\
\hline $\mathrm{C}$ & -4.68218 & -0.00467 & 3.25509 \\
\hline $\mathrm{H}$ & -5.65845 & -0.03879 & 2.76845 \\
\hline $\mathrm{C}$ & -5.27173 & 0.58160 & 0.35161 \\
\hline $\mathrm{C}$ & -6.22243 & -0.39077 & 0.02165 \\
\hline $\mathrm{H}$ & -5.92366 & -1.43704 & -0.06120 \\
\hline $\mathrm{C}$ & -7.55363 & -0.03099 & -0.18343 \\
\hline $\mathrm{H}$ & -8.28800 & -0.79732 & -0.44278 \\
\hline $\mathrm{C}$ & -7.94589 & 1.30019 & -0.05228 \\
\hline $\mathrm{H}$ & -8.98860 & 1.58217 & -0.21748 \\
\hline $\mathrm{C}$ & -7.00783 & 2.27028 & 0.30170 \\
\hline $\mathrm{H}$ & -7.31152 & 3.31339 & 0.41515 \\
\hline $\mathrm{C}$ & -5.67755 & 1.91488 & 0.50953 \\
\hline $\mathrm{H}$ & -4.95414 & 2.68208 & 0.78942 \\
\hline $\mathrm{C}$ & -3.52876 & -2.57777 & -0.00519 \\
\hline $\mathrm{C}$ & -3.13808 & -3.49692 & 0.99920 \\
\hline $\mathrm{C}$ & -3.60199 & -4.81458 & 0.93563 \\
\hline $\mathrm{H}$ & -3.30833 & -5.51706 & 1.72055 \\
\hline $\mathrm{C}$ & -4.41288 & -5.25209 & -0.10279 \\
\hline $\mathrm{H}$ & -4.77107 & -6.28380 & -0.12932 \\
\hline $\mathrm{C}$ & -4.73940 & -4.36709 & -1.12577 \\
\hline $\mathrm{H}$ & -5.34195 & -4.71938 & -1.96612 \\
\hline $\mathrm{C}$ & -4.30064 & -3.04243 & -1.10813 \\
\hline $\mathrm{C}$ & -2.17547 & -3.11214 & 2.10295 \\
\hline $\mathrm{H}$ & -1.95172 & -2.04679 & 1.96942 \\
\hline $\mathrm{C}$ & -0.85360 & -3.86970 & 1.97186 \\
\hline $\mathrm{H}$ & -0.98979 & -4.95501 & 2.10212 \\
\hline $\mathrm{H}$ & -0.13237 & -3.53124 & 2.73336 \\
\hline $\mathrm{H}$ & -0.39746 & -3.71484 & 0.98235 \\
\hline $\mathrm{C}$ & -2.77919 & -3.28880 & 3.49309 \\
\hline $\mathrm{H}$ & -3.70142 & -2.70046 & 3.60441 \\
\hline $\mathrm{H}$ & -2.07246 & -2.95563 & 4.26862 \\
\hline $\mathrm{H}$ & -3.02314 & -4.34424 & 3.69515 \\
\hline $\mathrm{C}$ & -4.56035 & -2.14452 & -2.30344 \\
\hline $\mathrm{H}$ & -4.50434 & -1.10630 & -1.94687 \\
\hline $\mathrm{C}$ & -3.45313 & -2.33092 & -3.34396 \\
\hline $\mathrm{H}$ & -2.45287 & -2.18167 & -2.90669 \\
\hline $\mathrm{H}$ & -3.56802 & -1.62325 & -4.18089 \\
\hline $\mathrm{H}$ & -3.47031 & -3.35331 & -3.75368 \\
\hline $\mathrm{C}$ & -5.92909 & -2.33640 & -2.94846 \\
\hline $\mathrm{H}$ & -6.02162 & -3.31851 & -3.43822 \\
\hline $\mathrm{H}$ & -6.09462 & -1.57183 & -3.72322 \\
\hline $\mathrm{H}$ & -6.74244 & -2.24992 & -2.21330 \\
\hline $\mathrm{C}$ & -2.69334 & 2.51702 & -0.28311 \\
\hline $\mathrm{C}$ & -2.29058 & 3.57214 & 0.57433 \\
\hline $\mathrm{C}$ & -2.48889 & 4.89498 & 0.17139 \\
\hline $\mathrm{H}$ & -2.18519 & 5.70451 & 0.83999 \\
\hline $\mathrm{C}$ & -3.05439 & 5.20079 & -1.06142 \\
\hline $\mathrm{H}$ & -3.20147 & 6.24132 & -1.35989 \\
\hline $\mathrm{C}$ & -3.41489 & 4.16609 & -1.91704 \\
\hline $\mathrm{H}$ & -3.84098 & 4.40321 & -2.89567 \\
\hline $\mathrm{C}$ & -3.24303 & 2.82777 & -1.55608 \\
\hline $\mathrm{C}$ & -1.68223 & 3.28161 & 1.92920 \\
\hline $\mathrm{H}$ & -1.43000 & 2.21224 & 1.92201 \\
\hline $\mathrm{C}$ & -0.39402 & 4.05956 & 2.18210 \\
\hline $\mathrm{H}$ & 0.35175 & 3.88232 & 1.39369 \\
\hline $\mathrm{H}$ & 0.05318 & 3.75812 & 3.14227 \\
\hline $\mathrm{H}$ & -0.56923 & 5.14578 & 2.23648 \\
\hline $\mathrm{C}$ & -2.70077 & 3.51160 & 3.04584 \\
\hline $\mathrm{H}$ & -3.00216 & 4.57110 & 3.08935 \\
\hline $\mathrm{H}$ & -2.27926 & 3.23672 & 4.02548 \\
\hline $\mathrm{H}$ & -3.60829 & 2.90743 & 2.89782 \\
\hline $\mathrm{C}$ & -3.60312 & 1.74614 & -2.55642 \\
\hline $\mathrm{H}$ & -3.54043 & 0.78266 & -2.02651 \\
\hline $\mathrm{C}$ & -2.59214 & 1.71637 & -3.70556 \\
\hline $\mathrm{H}$ & -2.63273 & 2.64948 & -4.28969 \\
\hline $\mathrm{H}$ & -2.79472 & 0.87778 & -4.39121 \\
\hline $\mathrm{H}$ & -1.55825 & 1.61783 & -3.33682 \\
\hline $\mathrm{C}$ & -5.03042 & 1.85550 & -3.08738 \\
\hline $\mathrm{H}$ & -5.76265 & 1.84310 & -2.26805 \\
\hline $\mathrm{H}$ & -5.25505 & 1.01114 & -3.75829 \\
\hline $\mathrm{H}$ & -5.18083 & 2.78183 & -3.66406 \\
\hline
\end{tabular}

(continued)

\begin{tabular}{|c|c|c|c|}
\hline $\mathrm{Tl}$ & 1.14385 & -0.39740 & 1.17160 \\
\hline $\mathrm{P}$ & 3.54951 & 0.11763 & -0.66615 \\
\hline $\mathrm{N}$ & 2.49716 & 1.18422 & -0.10068 \\
\hline $\mathrm{C}$ & 3.51738 & 0.06964 & -2.48310 \\
\hline $\mathrm{N}$ & 3.07441 & -1.25460 & 0.00698 \\
\hline $\mathrm{C}$ & 2.27772 & 0.11670 & -3.13525 \\
\hline $\mathrm{H}$ & 1.36237 & 0.17316 & -2.54308 \\
\hline $\mathrm{C}$ & 2.20091 & 0.09756 & -4.52238 \\
\hline $\mathrm{H}$ & 1.22494 & 0.13986 & -5.01232 \\
\hline $\mathrm{C}$ & 3.36903 & 0.03195 & -5.28447 \\
\hline $\mathrm{H}$ & 3.31220 & 0.02324 & -6.37551 \\
\hline $\mathrm{C}$ & 4.60653 & -0.02256 & -4.64845 \\
\hline $\mathrm{H}$ & 5.52431 & -0.07604 & -5.23869 \\
\hline $\mathrm{C}$ & 4.68212 & -0.00470 & -3.25512 \\
\hline $\mathrm{H}$ & 5.65840 & -0.03884 & -2.76850 \\
\hline $\mathrm{C}$ & 5.27172 & 0.58161 & -0.35166 \\
\hline $\mathrm{C}$ & 5.67753 & 1.91489 & -0.50956 \\
\hline $\mathrm{H}$ & 4.95411 & 2.68210 & -0.78940 \\
\hline $\mathrm{C}$ & 7.00782 & 2.27029 & -0.30177 \\
\hline $\mathrm{H}$ & 7.31150 & 3.31341 & -0.41520 \\
\hline $\mathrm{C}$ & 7.94590 & 1.30020 & 0.05215 \\
\hline $\mathrm{H}$ & 8.98860 & 1.58218 & 0.21733 \\
\hline $\mathrm{C}$ & 7.55365 & -0.03098 & 0.18328 \\
\hline $\mathrm{H}$ & 8.28802 & -0.79732 & 0.44258 \\
\hline $\mathrm{C}$ & 6.22244 & -0.39076 & -0.02176 \\
\hline $\mathrm{H}$ & 5.92368 & -1.43704 & 0.06106 \\
\hline $\mathrm{C}$ & 2.69336 & 2.51702 & 0.28312 \\
\hline $\mathrm{C}$ & 2.29057 & 3.57213 & -0.57430 \\
\hline $\mathrm{C}$ & 2.48890 & 4.89497 & -0.17136 \\
\hline $\mathrm{H}$ & 2.18518 & 5.70451 & -0.83995 \\
\hline $\mathrm{C}$ & 3.05446 & 5.20078 & 1.06143 \\
\hline $\mathrm{H}$ & 3.20155 & 6.24131 & 1.35988 \\
\hline $\mathrm{C}$ & 3.41498 & 4.16607 & 1.91702 \\
\hline $\mathrm{H}$ & 3.84110 & 4.40319 & 2.89564 \\
\hline $\mathrm{C}$ & 3.24309 & 2.82776 & 1.55607 \\
\hline $\mathrm{C}$ & 1.68216 & 3.28160 & -1.92914 \\
\hline $\mathrm{H}$ & 1.42993 & 2.21223 & -1.92194 \\
\hline $\mathrm{C}$ & 0.39392 & 4.05954 & -2.18198 \\
\hline $\mathrm{H}$ & 0.56911 & 5.14576 & -2.23636 \\
\hline $\mathrm{H}$ & -0.05333 & 3.75810 & -3.14211 \\
\hline $\mathrm{H}$ & -0.35181 & 3.88230 & -1.39352 \\
\hline $\mathrm{C}$ & 2.70063 & 3.51161 & -3.04583 \\
\hline $\mathrm{H}$ & 3.60817 & 2.90745 & -2.89785 \\
\hline $\mathrm{H}$ & 2.27907 & 3.23672 & -4.02545 \\
\hline $\mathrm{H}$ & 3.00201 & 4.57111 & -3.08936 \\
\hline $\mathrm{C}$ & 3.60322 & 1.74612 & 2.55640 \\
\hline $\mathrm{H}$ & 3.54051 & 0.78265 & 2.02648 \\
\hline $\mathrm{C}$ & 2.59229 & 1.71635 & 3.70558 \\
\hline $\mathrm{H}$ & 1.55838 & 1.61782 & 3.33688 \\
\hline $\mathrm{H}$ & 2.79489 & 0.87776 & 4.39122 \\
\hline $\mathrm{H}$ & 2.63292 & 2.64946 & 4.28971 \\
\hline $\mathrm{C}$ & 5.03054 & 1.85547 & 3.08729 \\
\hline $\mathrm{H}$ & 5.18099 & 2.78179 & 3.66397 \\
\hline $\mathrm{H}$ & 5.25520 & 1.01110 & 3.75819 \\
\hline $\mathrm{H}$ & 5.76274 & 1.84306 & 2.26793 \\
\hline $\mathrm{C}$ & 3.52876 & -2.57777 & 0.00520 \\
\hline $\mathrm{C}$ & 3.13805 & -3.49693 & -0.99917 \\
\hline $\mathrm{C}$ & 3.60197 & -4.81459 & -0.93561 \\
\hline $\mathrm{H}$ & 3.30829 & -5.51707 & -1.72052 \\
\hline $\mathrm{C}$ & 4.41291 & -5.25208 & 0.10278 \\
\hline $\mathrm{H}$ & 4.77111 & -6.28379 & 0.12931 \\
\hline $\mathrm{C}$ & 4.73945 & -4.36708 & 1.12575 \\
\hline $\mathrm{H}$ & 5.34203 & -4.71936 & 1.96608 \\
\hline $\mathrm{C}$ & 4.30068 & -3.04242 & 1.10812 \\
\hline $\mathrm{C}$ & 2.17540 & -3.11216 & -2.10289 \\
\hline $\mathrm{H}$ & 1.95166 & -2.04681 & -1.96938 \\
\hline $\mathrm{C}$ & 0.85354 & -3.86971 & -1.97175 \\
\hline $\mathrm{H}$ & 0.39743 & -3.71484 & -0.98222 \\
\hline $\mathrm{H}$ & 0.13228 & -3.53127 & -2.73322 \\
\hline $\mathrm{H}$ & 0.98972 & -4.95503 & -2.10199 \\
\hline $\mathrm{C}$ & 2.77907 & -3.28885 & -3.49305 \\
\hline $\mathrm{H}$ & 3.02301 & -4.34429 & -3.69511 \\
\hline $\mathrm{H}$ & 2.07232 & -2.95570 & -4.26856 \\
\hline $\mathrm{H}$ & 3.70131 & -2.70052 & -3.60442 \\
\hline $\mathrm{C}$ & 4.56040 & -2.14450 & 2.30342 \\
\hline $\mathrm{H}$ & 4.50435 & -1.10628 & 1.94685 \\
\hline $\mathrm{C}$ & 3.45322 & -2.33094 & 3.34398 \\
\hline $\mathrm{H}$ & 3.47045 & -3.35333 & 3.75370 \\
\hline $\mathrm{H}$ & 3.56813 & -1.62326 & 4.18090 \\
\hline $\mathrm{H}$ & 2.45295 & -2.18172 & 2.90675 \\
\hline $\mathrm{C}$ & 5.92917 & -2.33634 & 2.94839 \\
\hline $\mathrm{H}$ & 6.74249 & -2.24983 & 2.21320 \\
\hline $\mathrm{H}$ & 6.09470 & -1.57177 & 3.72314 \\
\hline $\mathrm{H}$ & 6.02175 & -3.31845 & 3.43814 \\
\hline
\end{tabular}




\subsection{References}

[S1] A. Stasch, Chem. Eur. J. 2012, 18, 15105-15112.

[S2] A. Stasch, Eur. J. Inorg. Chem. 2015, 258-270.

[S3] G. R. Fulmer, A. J. M. Miller, N. H. Sherden, H. E. Gottlieb, A. Nudelman, B. M. Stoltz, J. E. Bercaw, K. I. Goldberg, Organometallics 2010, 29, 2176-2179.

[S4] T. M. McPhillips, S. E. McPhillips, H. J. Chiu, A. E. Cohen, A. M. Deacon, P. J. Ellis, E. Garman, A. Gonzalez, N. K. Sauter, R. P. Phizackerley, S. M. Soltis, P. Kuhn, J. Synchrotron Radiat. 2002, 9, 401-406.

[S5] W. Kabsch, J. Appl. Crystallogr. 1993, 26, 795-800.

[S6] G. M. Sheldrick, Acta Cryst. 2015, C 71, 3-8.

[S7] A. L. Spek, Acta Cryst. 2015, C 71, 9-18.

[S8] C. Adamo, V. Barone, J. Chem. Phys. 1999, 110, 6158-6159.

[S9] F. Weigend, R. Ahlrichs, Phys. Chem. Chem. Phys. 2005, 7, 3297-3305.

[S10] S. Grimme, S. Ehrlich and L. Goerigk, J. Comp. Chem. 2011, 32, 1456-65.

[S11] Gaussian 09, Revision D.01: M. J. Frisch, G. W. Trucks, H. B. Schlegel, G. E. Scuseria, M. A. Robb, J. R. Cheeseman, G. Scalmani, V. Barone, B. Mennucci, G. A. Petersson, H. Nakatsuji, M. Caricato, X. Li, H. P. Hratchian, A. F. Izmaylov, J. Bloino, G. Zheng, J. L. Sonnenberg, M. Hada, M. Ehara, K. Toyota, R. Fukuda, J. Hasegawa, M. Ishida, T. Nakajima, Y. Honda, O. Kitao, H. Nakai, T. Vreven, J. A. Montgomery, Jr., J. E. Peralta, F. Ogliaro, M. Bearpark, J. J. Heyd, E. Brothers, K. N. Kudin, V. N. Staroverov, R. Kobayashi, J. Normand, K. Raghavachari, A. Rendell, J. C. Burant, S. S. Iyengar, J. Tomasi, M. Cossi, N. Rega, J. M. Millam, M. Klene, J. E. Knox, J. B. Cross, V. Bakken, C. Adamo, J. Jaramillo, R. Gomperts, R. E. Stratmann, O. Yazyev, A. J. Austin, R. Cammi, C. Pomelli, J. W. Ochterski, R. L. Martin, K. Morokuma, V. G. Zakrzewski, G. A. Voth, P. Salvador, J. J. Dannenberg, S. Dapprich, A. D. Daniels, Ö. Farkas, J. B. Foresman, J. V. Ortiz, J. Cioslowski, D. J. Fox, Gaussian, Inc., Wallingford CT, 2009.

[S12] a) D. Feller, J. Comp. Chem. 1996, 17, 1571-1586; b) K. L. Schuchardt, B. T. Didier, T. Elsehagen, S. L., V. Gurumoorthi, J. Chase, J. Li, T. L. Windus, J. Chem. Inf. Model. 2007, 47, 1045-1052.

[S13] NBO 6.0: E. D. Glendening, J, K. Badenhoop, A. E. Reed, J. E. Carpenter, J. A. Bohmann, C. M. Morales, C. R. Landis, F. Weinhold, Theoretical Chemistry Institute, University of Wisconsin, Madison (2013); http://nbo6.chem.wisc.edu/. 